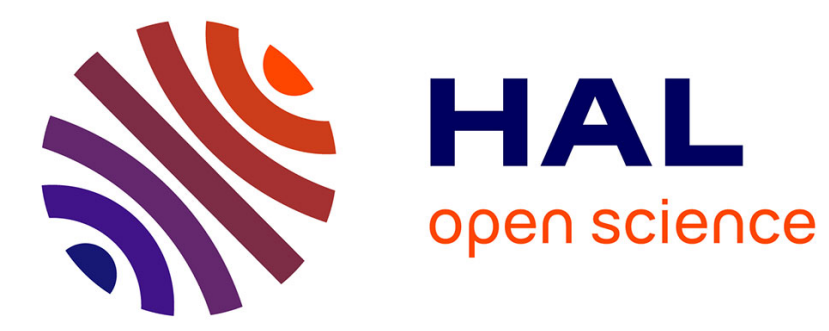

\title{
Orders for Simplifying Partial Partitions
}

\author{
Christian Ronse
}

\section{To cite this version:}

Christian Ronse. Orders for Simplifying Partial Partitions. Journal of Mathematical Imaging and Vision, 2017, 58 (3), pp.382-410. 10.1007/s10851-017-0717-5 . hal-02882867

\section{HAL Id: hal-02882867 https://hal.science/hal-02882867}

Submitted on 6 Nov 2020

HAL is a multi-disciplinary open access archive for the deposit and dissemination of scientific research documents, whether they are published or not. The documents may come from teaching and research institutions in France or abroad, or from public or private research centers.
L'archive ouverte pluridisciplinaire HAL, est destinée au dépôt et à la diffusion de documents scientifiques de niveau recherche, publiés ou non, émanant des établissements d'enseignement et de recherche français ou étrangers, des laboratoires publics ou privés. 


\title{
Orders for simplifying partial partitions
}

\author{
Christian Ronse
}

Received: date / Revised: date

\begin{abstract}
This paper is the third in a series devoted to orders on partial partitions in the framework of image analysis; the first two (with a view to image filtering and segmentation) identified 4 basic operations on blocks involved in such orders: merging, apportioning, creating and inflating blocks.

Here we consider orders where growing a partial partition decreases its support and diminishes the number of blocks. This can be done by a combination of merging (or apportioning) blocks with removing or deflating blocks (the opposite of creating or inflating blocks). We also introduce related operations on blocks: partial apportioning and partial merging.

The new orders that we obtain can be useful in relation to skeletonization, image simplification, for processing segmentation markers or to describe the evolution of object boundaries in hierarchies. There are also possible applications in geographic information processing.
\end{abstract}

\section{Introduction}

A partition of a set $E$ is a set of mutually disjoint nonempty subsets of $E$, whose union gives $E$; a partial partition of $E$ is a partition of any subset of $E$, in other words, a set of mutually disjoint non-empty subsets of $E$; the subsets of $E$ belonging to a (partial) partition are called blocks. The union of all blocks of a partial

This work received funding from the Agence Nationale de la Recherche, contract ANR-2010-BLAN-0205-01.

Christian Ronse

ICube, Université de Strasbourg, CNRS, 300 Boulevard Sébastien Brant, CS 10413, 67412 Illkirch Cedex, France E-mail: cronse@unistra.fr, URL: http://icube-miv.unistra.fr/ partition is called its support, and the complement of its support is called its background.

Partitions (or partial partitions) intervene routinely in image analysis. For instance image segmentation extracts from an image a partial partition whose blocks correspond to meaningful objects seen in the image. Now image filtering considers that a digital image is not simply made of pixels, but can be divided into zones (which are "homogeneous" in some sense), and filtering should improve the partition of zones by strenghtening meaningful zones at the expense of those considered as parasitic or irrelevant. Thus a partition-based approach to image filtering could be: (a) partition the image; (b) improve the partition; (c) modify the pixel values with the help of the context given by the improved partial partition. This is more precise than the older way of devising a filter independently of partitions, then experimentally watching its effect on partitions. Such a partition-based approach could be applied to other operations in image processing: noise cleaning, enhancement, skeletonization, etc.

The improvement relation "is at least better than" between partitions (or partial partitions) is obviously reflexive and transitive (it is a quasi-order). We will suppose that it is also antisymmetric, so that it is a partial order relation, in brief, an order. Hence we investigate orders on partial partitions.

For a long time, beside the identity, the only known order on partitions has been the classical refinement order [11]. It has been extended to partial partitions [6,7, 12], we call it the standard order [14]. Then Serra [20, 21] defined the building order on partial partitions; it also induces an order on partitions. In $[15,16]$ we introduced eight new orders on partial partitions: the merging, inflating, inclusion, merging-inflating, inclusion-inflating, apportioning, apportioning-inflating and exten- 
ded orders; we will describe them briefly in Subsection 2.2 .

\subsection{Basic operations on partial partitions}

In $[15,16]$ we showed how the growth of a partial partition could be expressed by a combination of elementary operations, namely:

- Creating new blocks (from background points); the opposite operation is removing blocks.

- Inflating individual blocks (adding to them background points); the opposite operation is deflating blocks (removing some but not all of their points).

- Merging blocks; the opposite operation is splitting blocks.

- Apportioning blocks [16]: some blocks can be split into parts, and each part is merged with another block; this operation generalizes merging, it was implicitly considered by Serra [20,21].

Each operation has an effect on the number of blocks, their size and the support:

- Creating new blocks increases the number of blocks and the support, but does not modify the size of existing blocks.

- Inflating blocks increases the size of blocks and the support, but does not modify the number of blocks.

- Merging or apportioning blocks increases the size of blocks, decreases the number of blocks, but does not modify the support.

In $[15,16]$, as our aim was to obtain larger blocks and a larger support, each of these 4 operations increased (or improved) the partial partition. We will now see that for some problems, this is not always the case.

\subsection{Image simplification}

There are several situations where the partial partition corresponding to an image is improved when the number of blocks and the support decrease. This is particular the case for operations aiming at image simplification.

Consider first the skeletonization of a binary image. Homotopic thinning decreases each connected component of the figure, in other words it deflates the blocks of the partial partition of connected components. Now the skeleton can contain spurious branches due to irregularities in the original figure, which must be removed [2, 4]. We reproduce in Figure 1 an example from [4]. In an iterative $3 \mathrm{D}$ thinning process based on the topological notion of an "isthmus" voxel, to each potential skeleton voxel one associates the range of time during which it is an isthmus, and by increasing the minimum length of this range, the skeleton is reduced, indeed spurious branches progressively disappear. This range length is called "persistence", and this notion is analoguous to the "saliency" of edges in hierarchical watershed segmentation $[9,10]$.

In a partition-based approach, the skeleton is segmented into branches and junction points, forming thus a partial partition. Then by analysing the blocks, some branches are marked as "spurious" and are removed; this can for instance be done on the basis of a parameter similar to the above persistence, for instance by comparison of the length of all branches. Thus here the skeleton is improved by removing blocks of the partition.

Now in practice, the segmentation of the skeleton can lead to oversegmentation: curved or irregular portions can be decomposed into several small branches, which must be merged. Thus the partition is improved by merging blocks.

A second example is given by the selection and processing of markers for region growing [1] or watershed segmentation [9]. One starts from an initial partial partition of markers, each one initiating a region, then the partial partition grows in order to reach the final segmentation. But before, it is necessary to process the markers in order to avoid some defects:

- When the marker for a region extends to a neighbouring region, the final segmentation will include in the main region part of the neighbouring one; to correct that defect, the marker must be reduced.

- Watershed segmentation tends to produce an oversegmentation; merging markers will merge the corresponding regions, thus diminishing the number of segmentation classes.

- When the region is "parasitic", i.e., it lies in the transition between two or more objects, it must be removed, and its contents should be apportioned between neighbouring regions; this can be achieved by removing the marker corresponding to that region.

Thus the partial partition made of all markers will be improved by deflating, merging or removing blocks.

Third, in the case of hierarchies $[9,10]$, to a growth of (partial) partitions corresponds an evolution of their boundaries, which can be modeled by an order relation. In particular when blocks are merged, the block boundaries are simplified: some boundary portions disappear, while others are merged. This will be discussed further in Subsection 3.3, see in particular Figure 9.

A fourth domain of application could be in geographic information processing, for instance in describing the relation between administrative subdivisions, 

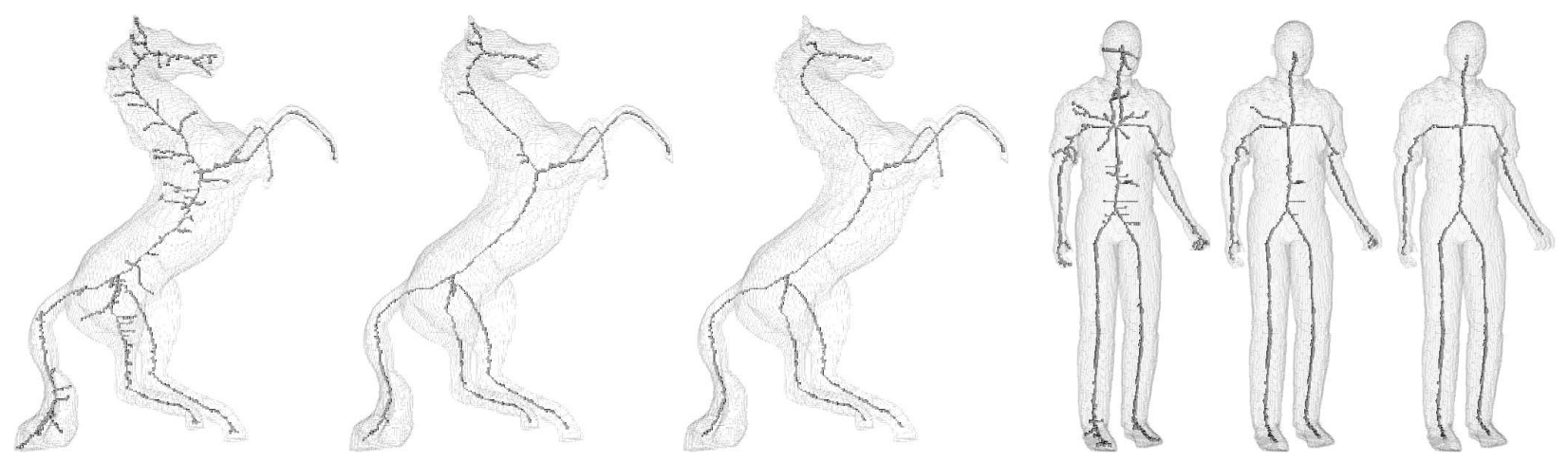

Fig. 1 From [4]: two examples of skeletons evolving by increasing the persistence threshold for isthmus voxels, leading to a progressive pruning of spurious branches.

the placement of resources and their allocation, see for instance in Section 4 the discussion around Figure 10.

For such problems we see that the operations of merging or apportioning blocks, by reducing the number of blocks while preserving the support, will improve the partial partition; on the other hand creating or inflating blocks will increase the support, and creating blocks will increase their numbers, thus they worsen the partial partition; hence the opposite operations, removing or deflating blocks, will improve the partial partition. Therefore we will define orders on partial partitions, where they grow by merging, apportioning, removing or deflating blocks.

\subsection{Our new orders}

Let us write $\Pi(E)$ for the set of all partitions of $E$, and $\Pi^{*}(E)$ for the set of all partial partitions of $E$. The union of all blocks of a partial partition $\pi$ is the support of $\pi$, written $\operatorname{supp}(\pi)$; thus $\pi$ is a partition of $\operatorname{supp}(\pi)$; the background of $\pi$ is the complement of the $\operatorname{support}, \operatorname{back}(\pi)=E \backslash \operatorname{supp}(\pi)$.

Important note: In order to ease understanding, in all our illustrations we show partial partitions with connected blocks. However no topological condition such as connectedness is required in our theory.

Our first order will be the regional order on $\Pi^{*}(E)$, where a partial partition grows by merging or removing blocks. In fact, all orders on $\Pi^{*}(E)$ that we have investigated contain the regional order. Now removing blocks can be seen as merging them with the background; hence, by adding to the space $E$ a point $\wp \notin E$ marking the background as a block, $\Pi^{*}(E)$ with the regional order turns out to be a complete lattice isomorphic to $\Pi(E \cup\{\wp\})$ ordered by refinement. See Figure 2 .

We will show that in a hierarchy of partitions of $E$ (that is, a chain of partitions for the refinement order), the partial partition of block boundaries evolves
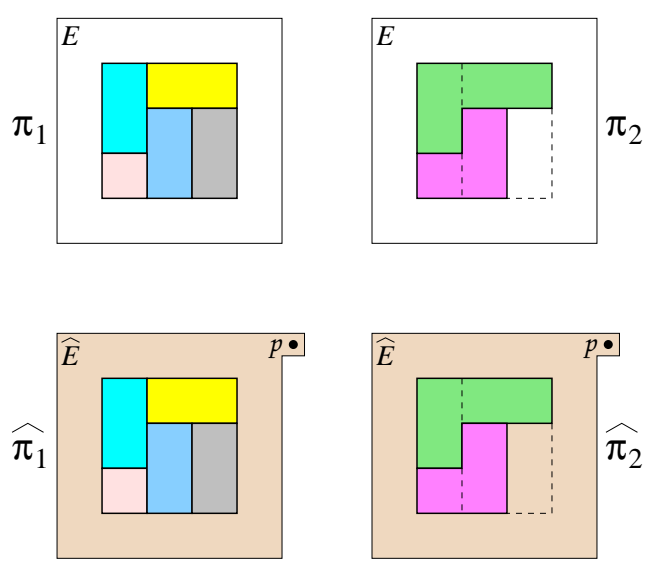

Fig. 2 Regional order. Top row: the background is in white, the blocks in colour; $\pi_{2}$ is obtained by merging some blocks of $\pi_{1}$ and removing some other blocks. Bottom row: we add to the space $E$ a point $\wp$ and transform each partial partition $\pi_{i}$ of $E(i=1,2)$ into a partition $\widehat{\pi}_{i}$ of $E \cup\{\wp\}$ by adding to it a block made of the background and $\wp$; then $\widehat{\pi_{2}}$ can be obtained from $\widehat{\pi_{1}}$ by merging blocks only.

according to the regional order. See Figure 9. One can also consider hierarchies of partial partitions for the regional order and describe the correponding evolution of block boundaries.

Our next order on $\Pi^{*}(E)$ is the linking order, where a partial partition grows by merging or deflating blocks; in particular, one can remove blocks by merging them with another then deflating the resulting block. Equivalently, the support of the partial partition decreases, and each block of the "smaller" partial partition overlaps at most one block of the "larger" (improved) one. See Figure 3.

We will see that the linking order can be used in geographic information processing (see Figure 10) and also to characterize the improvement of segmentation markers. 


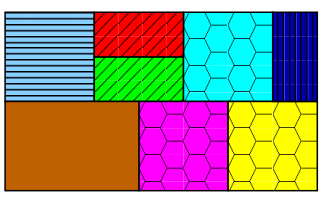

$\pi_{1}$

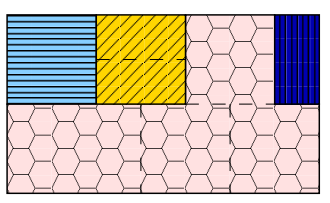

merge

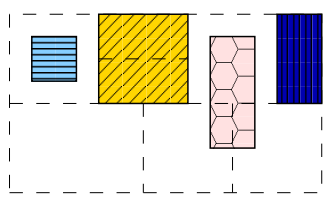

$\pi_{2}$

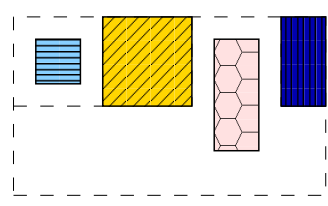

deflate
Fig. 3 Linking order. Top row: $\operatorname{supp}\left(\pi_{1}\right) \supseteq \operatorname{supp}\left(\pi_{2}\right)$, and each block of $\pi_{1}$ overlaps at most one block of $\pi_{2}$; we use the same hatching (but not the same colour) for overlapping blocks of $\pi_{1}$ and $\pi_{2}$, while a block of $\pi_{1}$ overlapping no block of $\pi_{2}$ has no hatching. Bottom row: $\pi_{2}$ can be obtained from $\pi_{1}$ by merging blocks, then by deflating blocks.
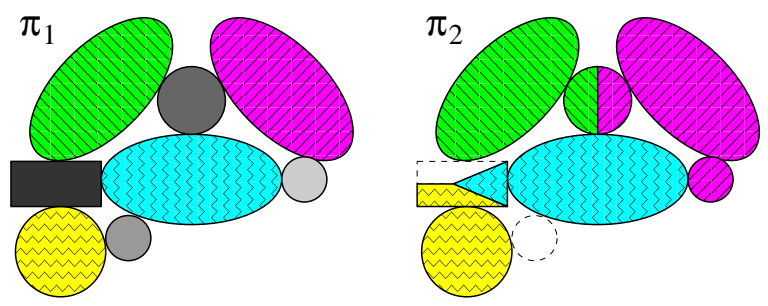

Fig. 4 Partial apportioning order. Left: in $\pi_{1}$ the blocks to be partially apportioned are in grey, while the preserved ones are in colour and hatched. Right: in the apportioned blocks, the discarded parts are shown dashed; the other parts are merged with preserved blocks, leading to $\pi_{2}$. Then $\operatorname{supp}\left(\pi_{1}\right) \supseteq$ $\operatorname{supp}\left(\pi_{2}\right)$, and each hatched block of $\pi_{2}$ contains the block of $\pi_{1}$ with the same hatching.

Next, we generalize block apportioning into partial apportioning: some blocks can be split into parts, some of the parts can be discarded (removed), and each remaining part is merged with another block; in particular, whole blocks can be merged or removed. Another view is that for the blocks split into parts, each part is merged either with the background or with a remaining block, so the block is apportioned in $\Pi(E \cup\{\wp\})$. An equivalent mathematical formulation is that the support of the partial partition decreases, and each block of the "larger" (improved) partial partition contains at least one block of the "smaller" one. See Figure 4. This gives the partial apportioning order on $\Pi^{*}(E)$.

In the same way as block apportioning was proposed in order to eliminate "parasitic" classes in an image segmentation partition [20,21], partial apportioning can be used for this purpose when the segmentation is a partial partition of objects against a background and some "parasitic" classes neighbour the background.
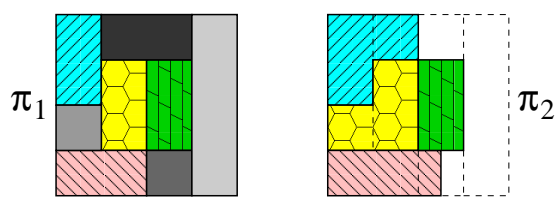

Fig. 5 Partial merging order. Left: in $\pi_{1}$ the blocks to be partially merged are in grey, while the preserved ones are in colour and hatched. Right: in the merged blocks, the discarded parts are shown dashed; the other parts are merged with preserved blocks, leading to $\pi_{2}$.

The above three orders are the most interesting. More orders on $\Pi^{*}(E)$ can be built by combining them, but their relevance is still tentative. The partial merging order on $\Pi^{*}(E)$ is the intersection of the linking and partial apportioning orders. It relies on the operation of partial merging, a generalization of block merging: some blocks are merged with others, or have only a part merged, the other part being discarded, or even are completely removed. See Figure 5. The difference with partial apportioning is the restriction that in an apportioned blocks, the part that does not go to the background must as a whole merge with a single block, we cannot have two or more parts merging with two or more blocks, as in Figure 4.

Finally, the linking and partial apportioning orders generate together the joining order, where a partial partition grows by apportioning or deflating blocks. Equivalently, given two partial partitions $\pi_{1}$ and $\pi_{2}, \pi_{1}$ is smaller than $\pi_{2}$ if $\operatorname{supp}\left(\pi_{1}\right) \supseteq \operatorname{supp}\left(\pi_{2}\right)$ and there is an injection $f: \pi_{2} \rightarrow \pi_{1}$ such that for any block $A \in \pi_{2}$, $f(A)$ overlaps no other block of $\pi_{2}$ than $A$, that is, $f(A) \cap \operatorname{supp}\left(\pi_{2}\right) \subseteq A$. See Figure 6 .

We have investigated other combinations, that of block apportioning with block removal or with partial merging. However this gives only special cases of partial apportioning, characterized by bizarre conditions, which seem useless in practice. Therefore we have not included them in this paper.

As the orders of $[15,16]$, each of our new orders will be defined in two ways: in terms of the operations on blocks that grow a partial partition, and by a predicate describing the relation between the blocks and supports of the two partial partitions.

As we did in $[15,16]$, for each order we will describe the covering relation, then show that these orders are graded and have a height function. This is important, since it means that progressively growing a partial partition, which can be done in several ways, always involves a fixed number of elementary steps. We will see that the regional, partial merging and partial apportioning orders have a simple covering relation and a simple grading, while the linking and joining orders have a 

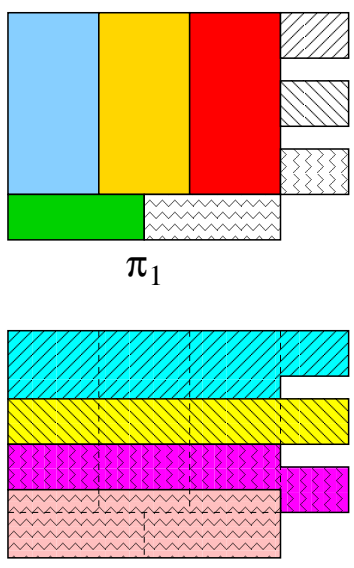

apportion

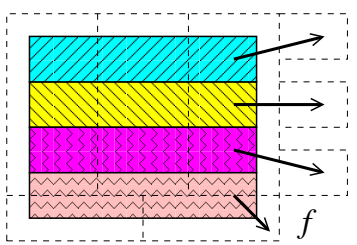

$\pi_{2}$

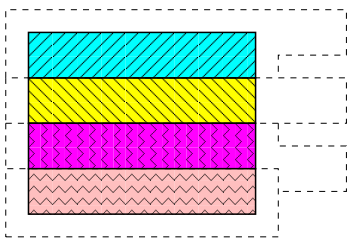

deflate

Fig. 6 Joining order. Top row: $\operatorname{supp}\left(\pi_{1}\right) \supseteq \operatorname{supp}\left(\pi_{2}\right)$, and arrows indicate the injection $f: \pi_{2} \rightarrow \pi_{1}$ associating to each $A \in \pi_{2}$ some $f(A) \in \pi_{1}$ such that $f(A) \cap \operatorname{supp}\left(\pi_{2}\right) \subseteq A$; each $f(A)$ is shown with the same hatching as $A$ (but without colour), while other blocks of $\pi_{1}$ are shown without hatching. Bottom row: first we apportion to the $f(A)\left(A \in \pi_{2}\right)$ all other blocks of $\pi_{1}$, then deflating blocks leads to $\pi_{2}$.

double covering and a double grading, as their growth involves two distinct elementary steps.

The present paper essentially terminates our investigation of orders on partial partitions initiated in [14] and developed in $[15,16]$. All our orders are built from operations on partial partitions that are meaningful in image analysis.

\section{Paper organization}

Section 2 recalls from $[15,16]$ the order-theoretic concepts needed, in particular grading, multi-grading and height, then the definitions of the orders studied there, both in terms of inclusion relations between blocks and operations on blocks. Section 3 is devoted to the regional order, which will be fundamental in the sequel. Then Section 4 studies the linking order, which relies for its definition on block overlap instead of block inclusion. Section 5 introduces the partial apportioning and partial merging orders, obtained through an extension of the operation of block apportioning. Section 6 considers the joining order, which is the most complicated of all. Finally, Section 7 discusses our results and concludes. Appendix A develops further the block overlap properties used in Section 3.

Table 1 lists the basic operations on blocks of partial partitions. Table 2 summarizes the definition and notation of all relations and orders on partial partitions considered in this paper.

\section{Mathematical preliminaries}

We summarize here the main concepts and results from $[15,16]$, with a few new facts. Subsection 2.1 describes combinations of orders, grading, height functions and multiple grading. Subsection 2.2 recalls the definition of the known orders on partial partitions.

We follow the notation and terminology from $[15$, 16]. In mathematical formulas, we will write " $\&$ " for the logical "and". For a set $A$, we will write $\mathcal{P}(A)$ for the set of parts of $A$, and $|A|$ for the cardinal of $A$. Given two subsets $A$ and $B$ of a set $E$, we say that $A$ and $B$ overlap and write $A \gamma B$, if $A \cap B \neq \emptyset$.

Every binary relation $R$ is identified with the set of ordered pairs $(a, b)$ such that $a R b$; thus the inclusion $R \subseteq S$ means that $(a R b) \Rightarrow(a S b)$, and the union $R \cup S$ is given by $a(R \cup S) b \Leftrightarrow(a R b)$ or $(a S b)$. The composition $R \cdot S$ of two relations is defined by $a R \cdot S$ $b \Leftrightarrow \exists c,(a R c) \&(c S b)$. The transitive closure of $R$ (least transitive relation containing $R$ ) is given by the union of all $R^{n}, n \geq 1$ (defined by recursion: $R^{1}=R$ and $R^{n+1}=R \cdot R^{n}$ ).

\subsection{Orders, grading and height}

We will consider several distinct (partial) order relations, they will be identified by a superscript on the usual order symbol, thus we write $\stackrel{x}{\leq}, \stackrel{x}{<}, \stackrel{x}{\geq}$ and $\stackrel{x}{>}$ for "less than or equal to", "strictly less than", "greater than or equal to" and "strictly greater than" respectively. The only exception will be for the building order on partial partitions, where we use $\Subset$ for "less than or equal to" and $\ni$ for "greater than or equal to", without any specific symbol for "strictly less than" and "strictly greater than". The covering relation associated to an order $\stackrel{x}{\leq}$ will generally be written $\stackrel{x}{\prec}$.

The set of all partial order relations on a set is closed under non-void intersection, but it is not a lattice. A quasi-order is a reflexive and transitive binary relation. An intersection of quasi-orders and the transitive closure of a union of quasi-orders, are quasi-orders, thus the set of all quasi-orders on a set constitutes a lattice. Also the intersection of an order and a quasi-order is an order.

Lemma 1 Let $\stackrel{\imath}{\leq}(\imath \in I)$ be partial orders on a set $P$, and let $\leq$ be the transitive closure of their union. Then $\leq$ is a quasi-order. Furthermore, there is a partial order on $P$ containing all $\iota^{\imath}(\imath \in I)$ if and only if $\leq$ is antisymmetric, and then $\leq$ is the least partial order on $P$ containing all $\stackrel{\imath}{\leq}(\iota \in I)$; given the covering relations 
Table 1 Basic operations on blocks of partial partitions. Top: those defined in $[15,16]$; bottom: new ones defined in this paper.

\begin{tabular}{l|l} 
Name & Definition \\
\hline $\begin{array}{l}\text { creating } \\
\text { inflating } \\
\text { deflating } \\
\text { merging }\end{array}$ & $\begin{array}{l}\text { new blocks are made from background points } \\
\text { some blocks are removed, their points go into the background } \\
\text { some background points are added to some blocks, growing them } \\
\text { some (but not all) points are removed from some blocks and go into the background } \\
\text { blocks are partitioned into groups, and in each group the blocks join together to make a new } \\
\text { block } \\
\text { some blocks are marked "apportioned", the others (at least one) are marked "remaining"; } \\
\text { the "apportioned" blocks are split into one or several parts, and each part is merged with a } \\
\text { "remaining" block }\end{array}$ \\
\hline partial apportioning & $\begin{array}{l}\text { some blocks are marked "apportioned", the others (at least one) are marked "remaining"; the } \\
\text { "apportioned" blocks are split into one or several parts, and each part is either merged with a } \\
\text { "remaining" block or removed (going into the background) } \\
\text { some blocks are marked "merged", the others (at least one) are marked "remaining"; the } \\
\text { "merged" blocks either are merged with a "remaining" block, or removed (going into the back- } \\
\text { ground), or split into two parts, one merged with a "remaining" block, the other removed }\end{array}$
\end{tabular}

$\stackrel{\imath}{\prec}$ and $\prec$ corresponding to $\stackrel{\imath}{\leq}(\imath \in I)$ and $\leq$, for any $x, y \in P$ with $x \prec y$, there is some $\imath \in I$ with $x \prec y$.

Proof The reflexivity of the $\stackrel{\imath}{\leq}$ is preserved by union and transitive closure, and by definition the transitive closure is transitive; thus $\leq$ is a quasi-order. If there is an order $\stackrel{*}{\leq}$ containing all $\leq^{\imath}(\imath \in I)$, it contains their union, and being transitive, it must contain the transitive closure of that union, that is, $\leq$; then for $x, y \in P$, if $x \leq y$ and $y \leq x$, we deduce that $x \stackrel{*}{\leq} y$ and $y \stackrel{*}{\leq} x$, hence $x=y$ by the antisymmetry of ${ }^{*}$; thus $\leq$ is antisymmetric. Conversely, if $\leq$ is antisymmetric, then it is a partial order containing all $\stackrel{\imath}{\leq}$, and as seen above, any other partial order $\stackrel{*}{\leq}$ containing all $\stackrel{\imath}{\leq}$ must contain $\leq$; therefore $\leq$ must be the least partial order containing all $\stackrel{\imath}{\leq}$

Let $x \prec y$; by definition we have $x=z_{0} \stackrel{\imath_{1}}{\leq} z_{1} \ldots$ $z_{n-1} \stackrel{\imath_{\mathrm{n}}}{\leq} z_{n}=y$, with $\imath_{1}, \ldots, \imath_{n} \in I$. As $x \prec y$, we may not have $x<z<y$, thus there is exactly one $j \in\{1, \ldots, n\}$ with $z_{j-1} \stackrel{\imath_{j}}{<} z_{j}$, so $x=\ldots=z_{j-1} \stackrel{\imath_{j}}{<} z_{j}=$ $\ldots=y$, that is, $x \stackrel{\imath_{\mathrm{j}}}{<} y$; if we did not have $x \stackrel{\imath_{\mathrm{j}}}{\prec} y$, we would have $x \stackrel{\imath_{\mathrm{j}}}{<} z \stackrel{\imath_{\mathrm{j}}}{<} y$, hence $x<z<y$, contradicting $x \prec y$.

Lemma 2 Let $\stackrel{\alpha}{\leq}$ and $\stackrel{\beta}{\leq}$ be two partial orders on a set $P$, and let $\leq$ be the transitive closure of their union. Let $\sqsubseteq$ be a partial order on a set $Q$ and consider a map $f: P \rightarrow Q$ such that for any $x, y \in P$ we have

and

$$
x \stackrel{\alpha}{<} y \Longrightarrow f(x)=f(y)
$$

$$
x \stackrel{\beta}{<} y \Longrightarrow f(x) \sqsubset f(y) \text {. }
$$

Then $\leq i s$ a partial order on $P$ and for any $x, y \in P$,

$$
\text { and } \begin{aligned}
x \leq y & \Longrightarrow f(x) \sqsubseteq f(y) \\
x \leq y \leq & \Longleftrightarrow[x \leq y \& f(x)=f(y)] .
\end{aligned}
$$

Given the covering relations $\stackrel{\alpha}{\prec}$ and $\prec$ corresponding to $\stackrel{\alpha}{\leq}$ and $\leq$, for any $x, y \in P, x \stackrel{\alpha}{\prec} y \Rightarrow x \prec y$.

Proof Let $x \leq y$; by definition we have $x=z_{0} \stackrel{\imath_{1}}{\leq} z_{1} \cdots$ $z_{n-1} \stackrel{\iota_{\mathrm{n}}}{\leq} z_{n}=y$, with $\imath_{1}, \ldots, \imath_{n} \in\{\alpha, \beta\}$; each case $z_{j-1} \stackrel{\alpha}{\leq} z_{j}$ and $z_{j-1} \stackrel{\beta}{\leq} z_{j}$ gives $f\left(z_{j-1}\right) \sqsubseteq f\left(z_{j}\right)$ for $j=$ $1, \ldots, n$, hence $f(x) \sqsubseteq f(y)$. If $x \leq y$ and $f(x)=f(y)$, then for $j=1, \ldots, n$ we have $f\left(z_{j-1}\right)=f\left(z_{j}\right)$, hence we cannot have $z_{j-1} \stackrel{\beta}{<} z_{j}$, so $z_{j-1} \stackrel{\alpha}{\leq} z_{j}$, thus $x \stackrel{\alpha}{\leq} y$. If $x \leq y$ and $y \leq x$, then $f(x) \sqsubseteq f(y)$ and $f(y) \sqsubseteq f(x)$, that is, $f(x)=f(y)$, so the above gives $x \stackrel{\alpha}{\leq} y$ and $y \stackrel{\alpha}{\leq} x$, thus $x=y$. Therefore $\leq$ is antisymmetric, it is a partial order.

If $x \stackrel{\alpha}{\prec} y$ but $x \nprec y$, then we have $x<z<y$, so $f(x)=f(y)$ and $f(x) \sqsubseteq f(z) \sqsubseteq f(y)$, thus $f(x)=$ $f(z)=f(y)$, hence $x \stackrel{\alpha}{\leq} z \stackrel{\alpha}{\leq} y$, and as $x<z<y, x \stackrel{\alpha}{<}$ $z \stackrel{\alpha}{<} y$, contradicting $x \stackrel{\alpha}{\prec} y$. Therefore $x \stackrel{\alpha}{\prec} y \Rightarrow x \prec y$.

Let $\leq$ be a partial order relation on a set $P$. Given $x, y \in P$ with $x \leq y$, the length of the interval $[x, y]=$ $\{z \in P \mid x \leq z \leq y\}$ is the supremum of all integers $n$ with $x=z_{0}<\cdots<z_{n}=y$; when this length is finite (for instance when $P$ is finite), it is the greatest such $n$, and the sequence takes the form $x=z_{0} \prec \cdots \prec z_{n}=y$, we call it a covering chain between $x$ and $y$. When $P$ has a least element 0 , the height of $x \in P$ is the length of the interval $[0, x]$. When $P$ has no least element, but 
Table 2 First: binary relations on $\mathcal{P}(E)$; second: binary relations on $\Pi^{*}(E)$; third: partial order relations on $\Pi^{*}(E)$ studied in $[15,16]$; fourth; new partial order relations on $\Pi^{*}(E)$ introduced in this paper. Notation designates the mathematical notation; for a relation $R$, Definition defines the relation for $A, B \in \mathcal{P}(E)$ or $\pi_{1}, \pi_{2} \in \Pi^{*}(E)$.

\begin{tabular}{|c|c|c|}
\hline Notation & Name & Definition \\
\hline$\gamma$ & overlap & $A \cap B \neq \emptyset$ \\
\hline$\Leftarrow$ & $\begin{array}{l}\text { support inclusion } \\
\text { support containment } \\
\text { support equality } \\
\text { singularity }\end{array}$ & $\begin{array}{l}\operatorname{supp}\left(\pi_{1}\right) \subseteq \operatorname{supp}\left(\pi_{2}\right) \\
\operatorname{supp}\left(\pi_{1}\right) \supseteq \operatorname{supp}\left(\pi_{2}\right) \\
\operatorname{supp}\left(\pi_{1}\right)=\operatorname{supp}\left(\pi_{2}\right) \\
\forall B, B^{\prime} \in \pi_{1}, \forall C \in \pi_{2}:\left[B \subseteq C, B^{\prime} \subseteq C\right] \Rightarrow B=B^{\prime}\end{array}$ \\
\hline$\leq$ & standard order & $\forall B \in \pi_{1}, \exists C \in \pi_{2}: B \subseteq C$ \\
\hline$\Subset$ & building order & $\forall C \in \pi_{2}, \exists B \in \pi_{1}: B \subseteq C$ \\
\hline$\subseteq$ & inclusion order & $\pi_{1} \subseteq \pi_{2}$ \\
\hline$\stackrel{i}{\leq}$ & inflating order & $\pi_{1} \leq \pi_{2} \& \pi_{1} \Subset \pi_{2} \& \pi_{1} \Leftarrow \pi_{2}$ \\
\hline$\stackrel{m}{\leq}$ & merging order & $\pi_{1} \leq \pi_{2} \& \operatorname{supp}\left(\pi_{1}\right)=\operatorname{supp}\left(\pi_{2}\right)$ \\
\hline$\stackrel{i}{\subseteq}$ & inclusion-inflating order & $\pi_{1} \leq \pi_{2} \& \pi_{1} \Leftarrow \pi_{2}$ \\
\hline$\stackrel{m i}{\leq}$ & merging-inflating order & $\pi_{1} \leq \pi_{2} \& \pi_{1} \Subset \pi_{2}$ \\
\hline$\stackrel{a}{\leq}$ & apportioning order & $\pi_{1} \Subset \pi_{2} \& \operatorname{supp}\left(\pi_{1}\right)=\operatorname{supp}\left(\pi_{2}\right)$ \\
\hline$\stackrel{\text { ai }}{\leq}$ & apportioning-inflating order & $\pi_{1} \Subset \pi_{2} \& \operatorname{supp}\left(\pi_{1}\right) \subseteq \operatorname{supp}\left(\pi_{2}\right)$ \\
\hline$\stackrel{e}{\leq}$ & extended order & $\pi_{1} \Subset \pi_{2} \cap \mathcal{P}\left(\operatorname{supp}\left(\pi_{1}\right)\right) \& \operatorname{supp}\left(\pi_{1}\right) \subseteq \operatorname{supp}\left(\pi_{2}\right)$ \\
\hline$\stackrel{r g}{\leq}$ & regional order & $\operatorname{supp}\left(\pi_{1}\right) \supseteq \operatorname{supp}\left(\pi_{2}\right) \& \pi_{1} \leq \pi_{2} \cup\left\{\operatorname{back}\left(\pi_{2}\right)\right\}$ \\
\hline$\stackrel{\ln }{\leq}$ & linking order & $\pi_{1} \vdash \pi_{2} \& \operatorname{supp}\left(\pi_{1}\right) \supseteq \operatorname{supp}\left(\pi_{2}\right)$ \\
\hline$\stackrel{\text { pa }}{\leq}$ & partial apportioning order & $\pi_{1} \Subset \pi_{2} \quad \& \operatorname{supp}\left(\pi_{1}\right) \supseteq \operatorname{supp}\left(\pi_{2}\right)$ \\
\hline$\leq$ & partial merging order & $\pi_{1} \Subset \pi_{2} \quad \& \pi_{1} \vdash \pi_{2} \quad \& \operatorname{supp}\left(\pi_{1}\right) \supseteq \operatorname{supp}\left(\pi_{2}\right)$ \\
\hline$\stackrel{\text { jn }}{\leq}$ & joining order & $\begin{array}{l}\operatorname{supp}\left(\pi_{1}\right) \supseteq \operatorname{supp}\left(\pi_{2}\right) \& \exists f: \pi_{2} \rightarrow \pi_{1} \text { injection, } \\
\forall A \in \pi_{2}, f(A) \cap \operatorname{supp}\left(\pi_{2}\right) \subseteq A\end{array}$ \\
\hline
\end{tabular}

for every $x \in P$ there exists a minimal element $m$ such that $m \leq x$, we call the height of $x$ w.r.t. $m$ the length of the interval $[m, x]$. Given a map $g: P \rightarrow \mathbf{Z}$, we say that $P$ is graded by $g[5,8]$ if for any $x, y \in P$, $x<y \Rightarrow g(x)<g(y)$ and $x \prec y \Rightarrow g(y)=g(x)+1$.

In [15] we generalized grading as follows. Let the covering relation $\prec$ be partitioned into $t$ mutually disjoint non-void relations $\stackrel{1}{\prec}, \ldots, \stackrel{\mathrm{t}}{\prec}$, and let $g_{1}, \ldots, g_{t}$ : $P \rightarrow \mathbf{Z}$. We consider the following three conditions:

1. For all $x, y \in P$ and $\imath=1, \ldots, t$ we have

$$
x \stackrel{\imath}{\prec} y \Longrightarrow\left\{\begin{array}{l}
g_{\imath}(y)=g_{\imath}(x)+1 \& \\
g_{\jmath}(y)=g_{\jmath}(x) \text { for } \jmath \neq \imath .
\end{array}\right.
$$

2. Every interval in $P$ has finite length.

3. For all $x, y \in P$,

$$
x<y \Longrightarrow \begin{cases}\forall \imath \in\{1, \ldots, t\}, & g_{\imath}(y) \geq g_{\imath}(x) \& \\ \exists \imath \in\{1, \ldots, t\}, & g_{\imath}(y)>g_{\imath}(x) .\end{cases}
$$

The conditions 1 and 2 together are equivalent to conditions 1 and 3 together. We say then that $P$ is graded by $\left(g_{1}, \ldots, g_{t}\right)$ for $(\stackrel{1}{\prec}, \ldots, \stackrel{\mathrm{t}}{\prec})$. We obtain then the following three consequences:

4. For all $x, y \in P$ and $\imath=1, \ldots, t$ we have

$$
x \stackrel{\imath}{\prec} y \Longleftrightarrow x \leq y \&\left\{\begin{array}{l}
g_{\imath}(y)=g_{\imath}(x)+1 \& \\
g_{\jmath}(y)=g_{\jmath}(x) \text { for } \jmath \neq \imath .
\end{array}\right.
$$

5. In a covering chain $z_{0} \prec \cdots \prec z_{n}$ in $P$, among the $n$ coverings $z_{\ell-1} \prec z_{\ell}(\ell=1, \ldots, n)$, there are $g_{\imath}\left(z_{n}\right)-$ $g_{\imath}\left(z_{0}\right)$ occurrences of $z_{\ell-1} \stackrel{\imath}{\prec} z_{\ell}$ for $\imath=1, \ldots, t$.

6. $P$ is graded by $\sum_{i=1}^{t} g_{\imath}$.

In the particular case where $t=1$, the fact that $P$ is graded by $g$ for $\prec$ simply means that $P$ is graded by $g$ (in the usual sense).

\subsection{Orders on partial partitions}

We write $\Pi(E)$ for the set of all partitions of $E$, and $\Pi^{*}(E)$ for the set of all partial partitions of $E$; thus $\Pi^{*}(E)$ is the disjoint union of all $\Pi(A)$ for $A \in \mathcal{P}(E)$. Write $\varnothing$ for the empty partial partition (with no block); 
then $\Pi(\emptyset)=\Pi^{*}(\emptyset)=\{\emptyset\}$. Set $\mathbf{1}_{\emptyset}=\mathbf{0}_{\emptyset}=\emptyset$, while for any $A \in \mathcal{P}(E) \backslash\{\emptyset\}$, let $\mathbf{1}_{A}=\{A\}$ (the partition of $A$ into a single block) and $\mathbf{0}_{A}=\{\{p\} \mid p \in A\}$ (the partition of $A$ into its singletons); following [11], we call $\mathbf{0}_{A}$ the identity partition of $A$, and $\mathbf{1}_{A}$ the universal partition of $A$. For $\pi \in \Pi^{*}(E)$, the support of $\pi$ is the union of its blocks, $\operatorname{supp}(\pi)=\bigcup \pi$; the complement of the support is the background of $\pi, \operatorname{back}(\pi)=E \backslash$ $\operatorname{supp}(\pi)$. Given $\pi \in \Pi^{*}(E)$ and $p \in E$, we define $\mathrm{Cl}_{\pi}(p)$, the class of $p$ in $\pi$ [12]: for $p \in \operatorname{back}(\pi)$ we set $\mathrm{Cl}_{\pi}(p)=$ $\emptyset$, while for $p \in \operatorname{supp}(\pi)$ we set $\mathrm{Cl}_{\pi}(p)=C$, where $p \in C \in \pi$ ( $C$ is the unique block of $\pi$ containing $p$ ).

We now recall the relations and partial orders on $\Pi^{*}(E)$ considered in $[15,16]$. The standard order $\leq$ and the building order $\Subset[20,21]$ are defined by setting for any $\pi_{1}, \pi_{2} \in \Pi^{*}(E)$ :

$$
\begin{aligned}
& \pi_{1} \leq \pi_{2} \Longleftrightarrow \forall B \in \pi_{1}, \exists C \in \pi_{2}, B \subseteq C, \\
& \pi_{1} \Subset \pi_{2} \Longleftrightarrow \forall C \in \pi_{2}, \exists B \in \pi_{1}, B \subseteq C .
\end{aligned}
$$

In other words, for $\pi_{1} \leq \pi_{2}$, every block of $\pi_{1}$ is included in a block of $\pi_{2}$, while for $\pi_{1} \Subset \pi_{2}$, every block of $\pi_{2}$ contains a block of $\pi_{1}$.

The standard order $\leq$ constitutes $\Pi^{*}(E)$ into a complete lattice with least element $\varnothing$ and greatest element $\mathbf{1}_{E}[12]$. Throughout this paper, the symbol $\wedge$ will designate the binary infimum for the standard order; for $\pi_{1}, \pi_{2} \in \Pi^{*}(E), \pi_{1} \wedge \pi_{2}$ is the set of all non-empty $B \cap C$ for $B \in \pi_{1}$ and $C \in \pi_{2}$. For $A \in \mathcal{P}(E)$ and $\pi \in \Pi^{*}(E)$, $\pi \wedge \mathbf{1}_{A}$ is the set of all non-void $B \cap A$ for $B \in \pi$.

The building order $\Subset$ is a partial order on $\Pi^{*}(E)$, but it does not make a lattice $[15,16,20,21]$.

Note that a block of $\pi_{1}$ is included in at most one block of $\pi_{2}$; on the other hand a block of $\pi_{2}$ can contain several blocks of $\pi_{1}$. We define thus the singularity relation $\Leftarrow$ : for $\pi_{1}, \pi_{2} \in \Pi^{*}(E)$, write $\pi_{1} \Leftarrow \pi_{2}\left(\right.$ or $\pi_{2} \Rightarrow \pi_{1}$ ) if every block of $\pi_{2}$ contains at most one block of $\pi_{1}$ :

$\pi_{1} \Leftarrow \pi_{2} \Longleftrightarrow\left(\begin{array}{l}\forall B, B^{\prime} \in \pi_{1}, \forall C \in \pi_{2}, \\ {\left[B \subseteq C \& B^{\prime} \subseteq C\right] \Rightarrow B=B^{\prime}}\end{array}\right)$.

In the sequel, we will use the following property given in $[15]$ :

$\forall \pi_{1}, \pi_{2} \in \Pi^{*}(E), \quad \pi_{1} \wedge \pi_{2} \Leftarrow \pi_{2} \Longrightarrow \pi_{1} \Leftarrow \pi_{2}$.

We now recall the definition of the 5 orders introduced in [15], both in terms of the relation between blocks of the two partial partitions and of the operations transforming the first into the second. Let $\pi_{1}, \pi_{2} \in$ $\Pi^{*}(E)$.

The inclusion order $\subseteq$ : for $\pi_{1} \subseteq \pi_{2}$, each block of $\pi_{1}$ is a block of $\pi_{2}$, thus $\pi_{2}$ is obtained from $\pi_{1}$ by adding new blocks made of points in $\operatorname{back}\left(\pi_{1}\right)$.
The inflating order $\stackrel{i}{\leq}$ :

$\pi_{1} \stackrel{i}{\leq} \pi_{2} \Longleftrightarrow\left[\pi_{1} \leq \pi_{2} \& \pi_{1} \Subset \pi_{2} \& \pi_{1} \Leftarrow \pi_{2}\right]$.

Here $\pi_{2}$ is obtained by inflating some blocks of $\pi_{1}$.

The merging order $\stackrel{\mathrm{m}}{\leq}$ :

$\pi_{1} \stackrel{\mathrm{m}}{\leq} \pi_{2} \Longleftrightarrow\left[\pi_{1} \leq \pi_{2} \& \operatorname{supp}\left(\pi_{1}\right)=\operatorname{supp}\left(\pi_{2}\right)\right]$.

Here $\pi_{2}$ is obtained by merging some blocks of $\pi_{1}$.

The inclusion-inflating order $\stackrel{\mathrm{i}}{\subseteq}$ :

$\pi_{1} \stackrel{\mathrm{i}}{\subseteq} \pi_{2} \Longleftrightarrow\left[\pi_{1} \leq \pi_{2} \& \pi_{1} \Leftarrow \pi_{2}\right]$.

Here $\pi_{2}$ is obtained from $\pi_{1}$ by inflating some blocks and/or adding new blocks. There is an alternate definition, see Proposition 13 of [15]:

$\pi_{1} \stackrel{\mathrm{i}}{\subseteq} \pi_{2} \Longleftrightarrow \pi_{2} \wedge \mathbf{1}_{\mathrm{supp}\left(\pi_{1}\right)}=\pi_{1}$

The merging-inflating order $\stackrel{\mathrm{mi}}{\leq}$ :

$\pi_{1} \stackrel{\mathrm{mi}}{\leq} \pi_{2} \Longleftrightarrow\left[\pi_{1} \leq \pi_{2} \& \pi_{1} \Subset \pi_{2}\right]$.

Here $\pi_{2}$ is obtained from $\pi_{1}$ by merging and/or inflating some blocks.

For the standard order, we have $\pi_{1} \leq \pi_{2}$ iff $\pi_{2}$ is obtained from $\pi_{1}$ by possibly adding new blocks then possibly merging some blocks.

Next we recall the definition of the 3 orders introduced in [16], using another operation on blocks, apportioning: some blocks can be split into parts, and each part is merged with a remaining block. Block merging is a special case of apportioning, where the apportioned blocks are "split into one part", that is, not split, before being merged to another block.

The apportioning order $\stackrel{\mathrm{a}}{\leq}$ :

$\pi_{1} \stackrel{\mathrm{a}}{\leq} \pi_{2} \Longleftrightarrow\left[\pi_{1} \Subset \pi_{2} \& \operatorname{supp}\left(\pi_{1}\right)=\operatorname{supp}\left(\pi_{2}\right)\right]$.

Here $\pi_{2}$ is obtained by apportioning some blocks of $\pi_{1}$.

The apportioning-inflating order $\stackrel{\text { ai }}{\leq}$ :

$\pi_{1} \stackrel{\text { ai }}{\leq} \pi_{2} \Longleftrightarrow\left[\pi_{1} \Subset \pi_{2} \& \operatorname{supp}\left(\pi_{1}\right) \subseteq \operatorname{supp}\left(\pi_{2}\right)\right]$.

Here $\pi_{2}$ is obtained from $\pi_{1}$ by apportioning and/or inflating some blocks.

The extended order $\stackrel{\mathrm{e}}{\leq}$ :

$\pi_{1} \stackrel{\mathrm{e}}{\leq} \pi_{2} \Longleftrightarrow\left[\begin{array}{l}\pi_{1} \Subset \pi_{2} \cap \mathcal{P}\left(\operatorname{supp}\left(\pi_{1}\right)\right) \\ \& \operatorname{supp}\left(\pi_{1}\right) \subseteq \operatorname{supp}\left(\pi_{2}\right)\end{array}\right]$.

Here $\pi_{2}$ is obtained from $\pi_{1}$ by possibly adding new blocks then possibly apportioning some blocks. 
Finally we recall the covering relations on $\Pi^{*}(E)$ associated to the above orders. Given $\pi_{1}, \pi_{2} \in \Pi^{*}(E)$, let us write:

$\pi_{1} \stackrel{\text { c }}{\prec} \pi_{2}$ if $\pi_{2}$ is obtained by adding a block to $\pi_{1}$ :

$\pi_{1} \stackrel{\mathrm{c}}{\prec} \pi_{2} \Longleftrightarrow\left[\begin{array}{l}\operatorname{supp}\left(\pi_{1}\right) \subset E, \exists B \subseteq \operatorname{back}\left(\pi_{1}\right), \\ B \neq \emptyset, \pi_{2}=\pi_{1} \cup\{B\}\end{array}\right] ;$

$\pi_{1} \stackrel{\mathrm{s}}{\prec} \pi_{2}$ if $\pi_{2}$ is obtained by adding a singleton block to $\pi_{1}$ :

$\pi_{1} \stackrel{\mathrm{s}}{\prec} \pi_{2} \Longleftrightarrow\left[\begin{array}{l}\operatorname{supp}\left(\pi_{1}\right) \subset E, \exists p \in \operatorname{back}\left(\pi_{1}\right), \\ \pi_{2}=\pi_{1} \cup\{\{p\}\}\end{array}\right] ;$

$\pi_{1} \stackrel{i}{\prec} \pi_{2}$ if $\pi_{2}$ is obtained by inflating one block of $\pi_{1}$ by exactly one point:

$\pi_{1} \stackrel{\text { i }}{\prec} \pi_{2} \Longleftrightarrow\left[\begin{array}{l}\operatorname{supp}\left(\pi_{1}\right) \subset E, \pi_{1} \neq \emptyset, \\ \exists p \in \operatorname{back}\left(\pi_{1}\right), \exists B \in \pi_{1}, \\ \pi_{2}=\left(\pi_{1} \backslash\{B\}\right) \cup\{B \cup\{p\}\}\end{array}\right]$.

$\pi_{1} \stackrel{\mathrm{m}}{\prec} \pi_{2}$ if $\pi_{2}$ is obtained by merging two blocks of $\pi_{1}$ :

$\pi_{1} \stackrel{\mathrm{m}}{\prec} \pi_{2} \Longleftrightarrow\left[\begin{array}{l}\left|\pi_{1}\right| \geq 2, \exists C_{1}, C_{2} \in \pi_{1}, C_{1} \neq C_{2}, \\ \pi_{2}=\left(\pi_{1} \backslash\left\{C_{1}, C_{2}\right\}\right) \cup\left\{C_{1} \cup C_{2}\right\}\end{array}\right] ;$

$\pi_{1} \stackrel{\mathrm{a}}{\prec} \pi_{2}$ if $\pi_{2}$ is obtained by apportioning a single block of $\pi_{1}$ among remaining blocks:

$\pi_{1} \stackrel{\mathrm{a}}{\prec} \pi_{2} \Longleftrightarrow\left[\begin{array}{l}\operatorname{supp}\left(\pi_{1}\right)=\operatorname{supp}\left(\pi_{2}\right) \&\left|\pi_{1}\right| \geq 2 \\ \& \exists A \in \pi_{1}, \pi_{1} \backslash\{A\} \stackrel{\mathrm{i}}{<} \pi_{2}\end{array}\right]$.

When $\pi_{1} \stackrel{\times}{\prec} \pi_{2}(x=c, s, i, m, a)$, we say that $\pi_{2}$ $x$-covers $\pi_{1}$, and we call the relation $\stackrel{\times}{\prec}$ the $x$-covering. The covering relations corresponding to the above orders are given here as a list of pairs order : covering:

$$
\begin{aligned}
& \subseteq: \stackrel{c}{\prec} \quad \stackrel{i}{\leq}: \stackrel{i}{\prec} \quad \stackrel{m}{\leq}: \stackrel{m}{\prec} \quad \stackrel{a}{\leq}: \stackrel{a}{\prec} \quad \stackrel{i}{\subseteq}: \stackrel{s}{\prec} \cup \stackrel{i}{\prec} \\
& \stackrel{\mathrm{mi}}{\leq}: \stackrel{\mathrm{m}}{\prec} \cup \stackrel{\mathrm{i}}{\prec} \quad \stackrel{\text { ai }}{\leq}: \stackrel{\mathrm{a}}{\prec} \cup \stackrel{\mathrm{i}}{\prec} \quad \leq: \stackrel{\mathrm{m}}{\prec} \cup \stackrel{\mathrm{s}}{\prec} \quad \stackrel{\mathrm{e}}{\leq}: \stackrel{\mathrm{a}}{\prec} \cup \stackrel{\mathrm{s}}{\prec}
\end{aligned}
$$

The first 4 orders are simple and have a simple covering, while the last 5 orders are compound and have a double covering.

Assume now that $E$ is finite. For any $\pi \in \Pi^{*}(E)$, we have defined its $c$-height $h_{c}(\pi)$, s-height $h_{s}(\pi)$, and $m$-height $h_{m}(\pi)$, as follows:

$$
\begin{gathered}
h_{c}(\pi)=|\pi|, \quad h_{s}(\pi)=|\operatorname{supp}(\pi)|, \\
h_{m}(\pi)=|\operatorname{supp}(\pi)|-|\pi| .
\end{gathered}
$$

We have $h_{m}(\pi)=h_{s}(\pi)-h_{c}(\pi), h_{s}(\pi)=h_{m}(\pi)+h_{c}(\pi)$ and $h_{c}(\pi)=h_{s}(\pi)-h_{m}(\pi)$. The following is straightforward:

Property 3 Let $E$ be finite. Then for any $\pi \in \Pi^{*}(E)$,
1. $0 \leq h_{c}(\pi) \leq|E|, h_{c}(\pi)=0 \Leftrightarrow \pi=\varnothing$ and $h_{c}(\pi)=$ $|E| \Leftrightarrow \pi=\mathbf{0}_{E}$

2. $0 \leq h_{s}(\pi) \leq|E|, h_{s}(\pi)=0 \Leftrightarrow \pi=\varnothing$ and $h_{s}(\pi)=$ $|E| \Leftrightarrow \pi \in \Pi(E)$

3. $0 \leq h_{m}(\pi) \leq|E|-1, h_{m}(\pi)=0 \Leftrightarrow \exists A \subseteq E, \pi=$ $\mathbf{0}_{A}$ and $h_{m}(\pi)=|E|-1 \Leftrightarrow \pi=\mathbf{1}_{E}$.

For the 4 simple orders, $\subseteq$ is graded by $h_{c}$ while $\stackrel{\text { i }}{\leq}$, $\stackrel{\mathrm{m}}{\leq}$ and $\stackrel{\mathrm{a}}{\leq}$ are graded by $h_{m}$. The 5 compound orders have the following double gradings:

$$
\begin{aligned}
& \stackrel{\mathrm{i}}{\subseteq}: \quad\left(h_{c}, h_{m}\right) \text { for }(\stackrel{\mathrm{s}}{\prec}, \stackrel{\mathrm{i}}{\prec}) \\
& \stackrel{\mathrm{mi}}{\leq}: \quad\left(-h_{c}, h_{s}\right) \text { for }(\stackrel{\mathrm{m}}{\prec, \stackrel{\mathrm{i}}{\prec})} \\
& \stackrel{\text { ai }}{\leq}:\left(-h_{c}, h_{s}\right) \text { for }(\stackrel{\text { a }}{\prec}, \stackrel{\mathrm{i}}{\prec}) \\
& \leq: \quad\left(h_{m}, h_{s}\right) \text { for }(\stackrel{\mathrm{m}}{\prec}, \stackrel{\mathrm{s}}{\prec}) \\
& \stackrel{\mathrm{e}}{\leq}: \quad\left(h_{m}, h_{s}\right) \text { for }(\stackrel{\mathrm{a}}{\prec}, \stackrel{\mathrm{s}}{\prec})
\end{aligned}
$$

In order to grade the new orders to be introduced in this paper, we define two new heights, which are complementary to $h_{c}$ and $h_{s}$. Let $E$ be finite; for any $\pi \in \Pi^{*}(E)$, we first define the $r$-height $h_{r}(\pi)$ by

$h_{r}(\pi)=|E|-h_{c}(\pi)=|E|-|\pi|$.

From item 1 of Property 3, we have $0 \leq h_{r}(\pi) \leq|E|$, $h_{r}(\pi)=0 \Leftrightarrow \pi=\mathbf{0}_{E}$ and $h_{r}(\pi)=|E| \Leftrightarrow \pi=\varnothing$. Note that for $\pi \in \Pi(E), h_{r}(\pi)=h_{m}(\pi)$ is the height of $\pi$ for the refinement order on $\Pi(E)$. Next we define the $b$-height $h_{b}(\pi)$ by

$h_{b}(\pi)=|E|-h_{s}(\pi)=|E|-|\operatorname{supp}(\pi)|=|\operatorname{back}(\pi)|$.

From item 2 of Property 3, we have $0 \leq h_{b}(\pi) \leq|E|$, $h_{b}(\pi)=0 \Leftrightarrow \pi \in \Pi(E)$ and $h_{b}(\pi)=|E| \Leftrightarrow \pi=\varnothing$.

\section{The regional order}

We will investigate the most basic way to simplify a partial partition: by merging or removing blocks. These operations will be involved in all orders considered in this paper, in other words these orders will all contain the regional order. We will formalize the intuition that removing a block amounts to merging it with the background, by adding a marker point to the background; thus the regional order for partial partitions will be isomorphic to the refinement order for partitions of the space with the point added. The regional order will be seen to have relations with hierarchies of partitions. 
3.1 A bijection between partial partitions and partitions

Given a partial partition $\pi$ of $E$, adding to it its background leads to a partition $\pi \cup\{\operatorname{back}(\pi)\}$ of $E$. This map is surjective but not injective, because in the resulting partition one cannot identify the block that was the background. Thus we will add to to the space $E$ a point $\wp \notin E$ that will mark the background; let $\widehat{E}=E \cup\{\wp\}$; hence to $\pi \in \Pi^{*}(E)$ one adds the block $\widehat{\operatorname{back}}(\pi)=\operatorname{back}(\pi) \cup\{\wp\}$, leading to

$\widehat{\pi}=\pi \cup\{\widehat{\operatorname{back}}(\pi)\} \in \Pi(\widehat{E})$.

Then the map $\pi \mapsto \widehat{\pi}$ is a bijection $\Pi^{*}(E) \rightarrow \Pi(\widehat{E})$; the inverse bijection removes from a partition of $\widehat{E}$ the block containing $\wp$, in other words, it is the map $\Pi(\widehat{E}) \rightarrow$ $\Pi^{*}(E): \pi \mapsto \pi \backslash\left\{\mathrm{Cl}_{\pi}(\wp)\right\}$, where $\mathrm{Cl}_{\pi}(\wp)$ designates the block of $\pi$ containing $\wp$.

This bijection has a combinatorial consequence. For $n \in \mathbf{N}$, the $n$-th Bell number $B_{n}$ [3] is the number of partitions of a set of size $n: B_{n}=|\Pi(E)|$ for $|E|=n$. Given the bijection $\Pi^{*}(E) \rightarrow \Pi(\widehat{E})$ and the fact that $\Pi^{*}(E)$ is the disjoint union of all $\Pi(A)$ for $A \in \mathcal{P}(E)$, it follows that

$$
|\Pi(\widehat{E})|=\sum_{A \in \mathcal{P}(E)}|\Pi(A)|,
$$

which leads to a well-known recursion formula for computing Bell numbers [18]:

$$
B_{n+1}=\sum_{k=0}^{n}\left(\begin{array}{l}
n \\
k
\end{array}\right) B(k) .
$$

Now we apply this bijection to translate to $\Pi^{*}(E)$ the refinement order on $\Pi(\widehat{E})$ :

Proposition 4 Let $\pi_{1}, \pi_{2} \in \Pi^{*}(E)$. Then the following statements are equivalent:

1. $\widehat{\pi_{1}} \leq \widehat{\pi_{2}}$ for the refinement order on $\Pi(\widehat{E})$.

2. $\operatorname{supp}\left(\pi_{1}\right) \supseteq \operatorname{supp}\left(\pi_{2}\right)$ and $\pi_{1} \leq \pi_{2} \cup\left\{\operatorname{back}\left(\pi_{2}\right)\right\}$ for the standard order (that is, every block of $\pi_{1}$ is included either in a block of $\pi_{2}$ or in the background of $\left.\pi_{2}\right)$.

3. Every block of $\pi_{2}$ is the union of some blocks of $\pi_{1}$.

Proof For all $A \in \pi_{2}$, define $\mathcal{B}_{A}=\left\{B \in \pi_{1} \mid B \gamma A\right\}$ and $\mathcal{C}_{A}=\left\{B \in \pi_{1} \mid B \subseteq A\right\}$.

$1 \Longrightarrow 2$ : Every block of $\widehat{\pi_{1}}=\pi_{1} \cup\left\{\widehat{\operatorname{back}}\left(\pi_{1}\right)\right\}$ is included in a block of $\widehat{\pi_{2}}=\pi_{2} \cup\left\{\widehat{\operatorname{back}}\left(\pi_{2}\right)\right\}$. Since $\operatorname{both} \widehat{\operatorname{back}}\left(\pi_{1}\right)$ and $\widehat{\operatorname{back}}\left(\pi_{2}\right)$ contain the point $\wp$, we have $\widehat{\operatorname{back}}\left(\pi_{1}\right) \subseteq \widehat{\operatorname{back}}\left(\pi_{2}\right)$, that is, $\operatorname{back}\left(\pi_{1}\right) \subseteq \operatorname{back}\left(\pi_{2}\right)$, thus $\operatorname{supp}\left(\pi_{1}\right) \supseteq \operatorname{supp}\left(\pi_{2}\right)$. Now let $A \in \pi_{1}$; then $A$ is included in a block of $\widehat{\pi_{2}}$, that is, $A$ is included either in a block of $\pi_{2}$, or in $\widehat{\operatorname{back}}\left(\pi_{2}\right)$, and as $\wp \notin A$, the latter means that $A \subseteq \operatorname{back}\left(\pi_{2}\right)$.

$2 \Longrightarrow 3: \quad$ Let $A \in \pi_{2}$. Since $\operatorname{supp}\left(\pi_{1}\right) \supseteq \operatorname{supp}\left(\pi_{2}\right)$, $A \subseteq \operatorname{supp}\left(\pi_{1}\right)$, hence $A \subseteq \cup \mathcal{B}_{A}$. For any $B \in \mathcal{B}_{A}, B$ is not included in back $\left(\pi_{2}\right)$, so it is included in a block of $\pi_{2}$, that is, $B \subseteq A$. Thus $\cup \mathcal{B}_{A} \subseteq A$, hence $A=\cup \mathcal{B}_{A}$. (In fact, $\mathcal{B}_{A}=\mathcal{C}_{A}$.)

$3 \Longrightarrow 1$ : For any $A \in \pi_{2}$, we have $A=\bigcup \mathcal{C}_{A}$ with $\mathcal{C}_{A} \subseteq \pi_{1}$, hence $A \subseteq \operatorname{supp}\left(\pi_{1}\right)$. It follows that $\operatorname{supp}\left(\pi_{2}\right) \subseteq \operatorname{supp}\left(\pi_{1}\right)$, thus $\operatorname{back}\left(\pi_{1}\right) \subseteq \operatorname{back}\left(\pi_{2}\right)$ and $\widehat{\operatorname{back}}\left(\pi_{1}\right) \subseteq \widehat{\operatorname{back}}\left(\pi_{2}\right)$. For any $B \in \pi_{1}$, either $B \subseteq$ $\operatorname{back}\left(\pi_{2}\right) \subseteq \widehat{\operatorname{back}}\left(\pi_{2}\right)$, or $B \gamma \operatorname{supp}\left(\pi_{2}\right)$, and then there is some $A \in \pi_{2}$ such that $B \gamma A$; now for $p \in B \cap A$, there is $C \in \mathcal{C}_{A}$ such that $p \in C$, so we deduce that $B=C$ and $B \in \mathcal{C}_{A}$, that is, $B \subseteq A$. We have thus shown that every block of $\widehat{\pi_{1}}=\pi_{1} \cup\left\{\widehat{\operatorname{back}}\left(\pi_{1}\right)\right\}$ is included in a block of $\widehat{\pi_{2}}=\pi_{2} \cup\left\{\widehat{\operatorname{back}}\left(\pi_{2}\right)\right\}$, therefore $\widehat{\pi_{1}} \leq \widehat{\pi_{2}}$.

We define thus the regional order $\stackrel{\mathrm{rg}}{\leq}$ on $\Pi^{*}(E)$ by setting $\pi_{1} \stackrel{\text { rg }}{\leq} \pi_{2}$ if any of the three equivalent conditions in Proposition 4 holds. See also Figure 2. This gives a precise basis for the idea that removing blocks can be seen as merging them with the background. Since the regional order on $\Pi^{*}(E)$ corresponds to the refinement order on $\Pi(\widehat{E})$ by the bijection $\Pi^{*}(E) \rightarrow \Pi(\widehat{E}): \pi \mapsto$ $\widehat{\pi}$, we deduce the following:

Theorem $5 \Pi^{*}(E)$ with the regional order $\stackrel{\mathrm{rg}}{\leq}$ is a complete lattice isomorphic to $\Pi(\widehat{E})$ ordered by refinement. The regional order contains the merging $\stackrel{m}{\leq}$ and inverse inclusion $\supseteq$ orders: for any $\pi_{1}, \pi_{2} \in \Pi^{*}(E)$, each of $\pi_{1} \stackrel{\mathrm{m}}{\leq} \pi_{2}$ and $\pi_{1} \supseteq \pi_{2}$ implies $\pi_{1} \stackrel{\mathrm{rg}}{\leq} \pi_{2}$. It is generated by composing merging and inverse inclusion in any order:

$$
\begin{aligned}
\pi_{1} \stackrel{\mathrm{rg}}{\leq} \pi_{2} & \Longleftrightarrow\left(\exists \pi_{3} \in \Pi^{*}(E), \pi_{1} \stackrel{\mathrm{m}}{\leq} \pi_{3} \supseteq \pi_{2}\right) \\
& \Longleftrightarrow\left(\exists \pi_{4} \in \Pi^{*}(E), \pi_{1} \supseteq \pi_{4} \leq \pi_{2}\right) .
\end{aligned}
$$

The greatest and least elements are $\varnothing$ and $\mathbf{0}_{E}$. The covering relation is $\stackrel{\mathrm{m}}{\prec} \cup \stackrel{\mathrm{c}}{\succ}$.

Let $E$ be finite. Then $\left(\Pi^{*}(E), \stackrel{\mathrm{rg}}{\leq}\right)$ is graded by $h_{r}$, or equivalently by $-h_{c}$, that is, for any $\pi_{1}, \pi_{2} \in \Pi^{*}(E)$ we have

$$
\begin{aligned}
& \pi_{1} \stackrel{\mathrm{rg}}{\underset{\mathrm{m}}{\gtrless} \pi_{2}} \Longrightarrow h_{c}\left(\pi_{1}\right)>h_{c}\left(\pi_{2}\right), \\
& \pi_{1} \stackrel{\gtrless}{\prec} \pi_{2} \Longrightarrow h_{c}\left(\pi_{2}\right)=h_{c}\left(\pi_{1}\right)-1, \\
& \pi_{1} \stackrel{\mathrm{c}}{\succ} \pi_{2} \Longrightarrow h_{c}\left(\pi_{2}\right)=h_{c}\left(\pi_{1}\right)-1 .
\end{aligned}
$$

For $\pi \in \Pi^{*}(E)$, the height of $\pi$ is $h_{r}(\pi)$.

Proof The isomorphism follows from item 1 of Proposition 4. If $\pi_{1} \stackrel{m}{\leq} \pi_{2}$, then $\operatorname{supp}\left(\pi_{1}\right)=\operatorname{supp}\left(\pi_{2}\right)$ and 

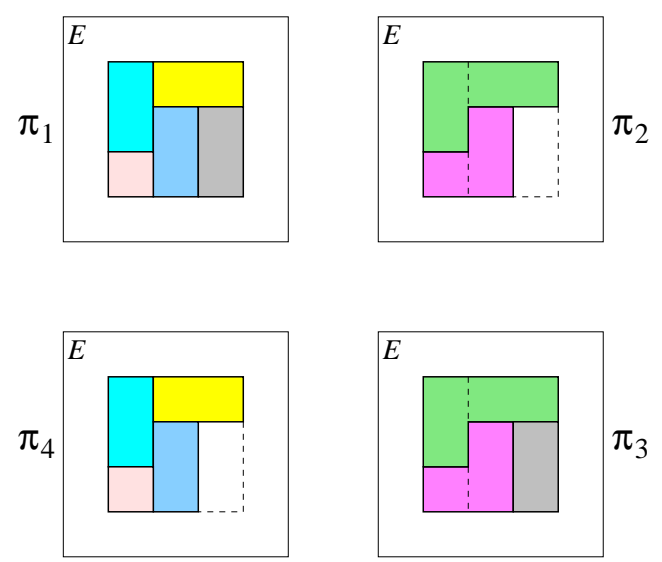

Fig. 7 For $\pi_{1} \stackrel{\text { rg }}{\leq} \pi_{2}$, given $\pi_{4}$ the set of blocks of $\pi_{1}$ included in blocks of $\pi_{2}$, we have $\pi_{1} \supseteq \pi_{4} \stackrel{\mathrm{m}}{\leq} \pi_{2}$; given $\pi_{0}$ the set of blocks removed from $\pi_{1}$ (here the grey block), $\pi_{3}=\pi_{0} \cup \pi_{2}$ satisfies $\pi_{1} \stackrel{\mathrm{m}}{\leq} \pi_{3} \supseteq \pi_{2}$.

$\pi_{1} \leq \pi_{2} \leq \pi_{2} \cup\left\{\operatorname{back}\left(\pi_{2}\right)\right\}$, so item 2 of Proposition 4 is satisfied; if $\pi_{1} \supseteq \pi_{2}$, then every block of $\pi_{2}$ is a block of $\pi_{1}$, so item 3 of Proposition 4 is satisfied; both cases lead to $\pi_{1} \stackrel{\mathrm{rg}}{\leq} \pi_{2}$.

If $\pi_{1} \stackrel{\mathrm{m}}{\leq} \pi_{3} \supseteq \pi_{2}$, then $\pi_{1} \stackrel{\mathrm{rg}}{\leq} \pi_{3} \stackrel{\mathrm{rg}}{\leq} \pi_{2}$; if $\pi_{1} \supseteq \pi_{4} \stackrel{\mathrm{m}}{\leq}$ $\pi_{2}$, then $\pi_{1} \stackrel{\mathrm{rg}}{\leq} \pi_{4} \underset{\mathrm{rg}}{\stackrel{\mathrm{rg}}{\leq}} \pi_{2} ;$ both cases lead to $\pi_{1} \stackrel{\mathrm{rg}}{\leq} \pi_{2}$. Conversely, let $\pi_{1} \leq \pi_{2}$. For every $A \in \pi_{2}$, there is some $\mathcal{C}_{A} \subseteq \pi_{1}$ such that $A=\bigcup \mathcal{C}_{A}$; let $\pi_{4}=\bigcup_{A \in \pi_{2}} \mathcal{C}_{A}$. Then $\pi_{1} \supseteq \pi_{4}$; now

$$
\operatorname{supp}\left(\pi_{4}\right)=\bigcup \pi_{4}=\bigcup_{A \in \pi_{2}} \bigcup \mathcal{C}_{A}=\bigcup_{A \in \pi_{2}} A=\operatorname{supp}\left(\pi_{2}\right)
$$

and every block $B \in \pi_{4}$ belongs to $\mathcal{C}_{A}$ for some $A$, so $B \subseteq A$, hence $\pi_{4} \stackrel{\mathrm{m}}{\leq} \pi_{2}$. Now let $\pi_{0}=\pi_{1} \backslash \pi_{4}$; since $\operatorname{supp}\left(\pi_{2}\right)=\operatorname{supp}\left(\pi_{4}\right)$, which is disjoint from $\operatorname{supp}\left(\pi_{0}\right)$, $\pi_{3}=\pi_{0} \cup \pi_{2}$ is a partial partition. As $\pi_{4} \stackrel{\mathrm{m}}{\leq} \pi_{2}, \pi_{1}=$ $\pi_{0} \cup \pi_{4} \stackrel{\mathrm{m}}{\leq} \pi_{0} \cup \pi_{2}=\pi_{3}$, and obviously $\pi_{3} \supseteq \pi_{2}$. The argument is illustrated in Figure 7.

The greatest element of $\Pi(\widehat{E})$ is $\mathbf{1}_{\widehat{E}}$, whose unique block contains $\wp$; removing that block, one obtains $\emptyset \in$ $\Pi^{*}(E)$, which is thus the greatest element for $\stackrel{\mathrm{rg}}{\leq}$. The least element of $\Pi(\widehat{E})$ is $\mathbf{0}_{\widehat{E}}$, whose block containing $\wp$ is the singleton $\{\wp\}$; removing that singleton, one obtains $\mathbf{0}_{E} \in \Pi^{*}(E)$, which is thus the least element for $\stackrel{\text { rg }}{\leq}$.

The covering relation in $\Pi(\widehat{E})$ is $\stackrel{\mathrm{m}}{\prec}$; for $\pi_{1}, \pi_{2} \in$ $\Pi^{*}(E), \widehat{\pi_{1}} \stackrel{\mathrm{m}}{\prec} \widehat{\pi_{2}}$ if $\widehat{\pi_{2}}$ is obtained by merging two blocks of $\widehat{\pi_{1}}$. If these two blocks are in $\pi_{1}$, then they are merged in $\Pi^{*}(E)$ and $\pi_{1} \stackrel{\mathrm{m}}{\prec} \pi_{2}$. If one of the blocks is $\widehat{\operatorname{back}}\left(\pi_{1}\right)$, the other one in $\pi_{1}$, this means that in $\Pi^{*}(E)$ the other block is merged with the background, that is, removed, and $\pi_{1} \stackrel{c}{\succ} \pi_{2}$.
Let $E$ be finite. Then $\Pi(\widehat{E})$, ordered by refinement, has height function $h_{m}$. Now $\forall \pi \in \Pi^{*}(E)$,

$$
\begin{aligned}
h_{m}(\widehat{\pi}) & =|\operatorname{supp}(\widehat{\pi})|-|\widehat{\pi}|=(|E|+1)-(|\pi|+1) \\
& =|E|-|\pi|=|E|-h_{c}(\pi)=h_{r}(\pi),
\end{aligned}
$$

cf. (3). Thus by isomorphism the height of $\pi$ in $\Pi^{*}(E)$ is $h_{r}(\pi)=|E|-h_{c}(\pi)$. Then $\Pi^{*}(E)$ is graded by that height, and as $|E|$ is constant, it is graded by $-h_{c}$.

Note that this order does not distinguish between block merging and block removal; for instance in a covering chain, the removal of $k$ blocks $(k>0)$ can be obtained by $t$ binary block mergings $\stackrel{\mathrm{m}}{\prec}$ (where $0 \leq t \leq$ $k-1$ ) followed by $k-t$ individual block removals $\stackrel{c}{\succ}$; for instance, for $t=0$ by $k$ individual block removals, and for $t=k-1$ by $k-1$ binary block mergings followed by the removal of the block resulting from the mergings. However the evolution of the support indicates the existence of block removals: for $\pi_{1} \stackrel{\text { rg }}{\leq} \pi_{2}$, we have $\operatorname{supp}\left(\pi_{1}\right) \supset \operatorname{supp}\left(\pi_{2}\right)$ if and only if at least one block of $\pi_{1}$ has been removed in order to obtain $\pi_{2}$.

\subsection{Possible applications}

As mentioned in the Introduction, see Figure 1, skeleton filtering $[2,4]$ can involve the removal of spurious branches and the merging of significant branches. Thus assuming that the skeleton is segmented into a partial partition of branches and junction points, the filtering will grow the partial partition according to the regional order.

In double thresholding of grey-level images [22], one applies two thresholdings, one by a narrow interval and another by a wide interval, then one keeps all connected components of the wide thresholding that contain a connected component of the narrow thresholding. Thus if one decomposes the image into three parts, (a) pixels satisfying the narrow thresholding, (b) those satisfying the wide thresholding but not the narrow one, and (c) those that do not satisfy the wide thresholding, then we eliminate all connected components of (c), keep all connected components of (a), but of the connected components of (b) we retain only those that are adjacent to a connected component of (a); the retained components (b) and (a) will then be regrouped by merging adjacent ones. We have thus a combination of block removals and block mergings, that is, a growth of the partial partition for the regional order.

We can apply something similar to a partial partition, for instance one obtained by segmentation of objects against a background in the presence of noise. One can measure in some way the quality of the blocks, and 


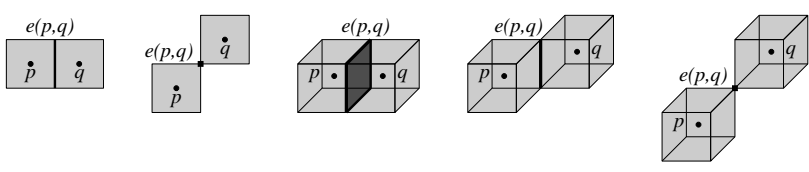

Fig. 8 In 2D, the edge element $e(p, q)$ between pixels $p$ and $q$ is $1 \mathrm{D}$ when $p$ and $q$ are axially adjacent, and 0D when $p$ and $q$ are diagonally adjacent. In 3D, the edge element $e(p, q)$ between voxels $p$ and $q$ is 2D when $p$ and $q$ are axially adjacent, 1D when $p$ and $q$ are diagonally adjacent, and OD when $p$ and $q$ are diametrically adjacent.

give a ternary rating, say high, medium or low. Blocks of high quality will be retained, those of low quality will be eliminated, while those of medium quality can be accepted only on certain conditions, and then they are merged with blocks of high quality.

Now we will see in the next subsection that the regional order is involved in the evolution of boundaries in a hierarchy of partitions.

\subsection{Hierarchies and boundaries}

A hierarchy is an increasing sequence of partitions for the refinement order, or of partial partitions for the standard order, going from the least to the greatest element of the lattice: $\pi_{0} \leq \ldots \leq \pi_{n}=\mathbf{1}_{E}$, where $\pi_{0}=\mathbf{0}_{E}$ for partitions but $\pi_{0}=\varnothing$ for partial partitions. Hierarchies have been extensively used in image segmentation [9] and filtering [19]. In Section 4.2 of [15] we studied edge saliency in a hierarchy of partial partitions, that is, the evolution of edges and block boundaries as one goes up in a hierarchy [10].

Assuming a digital framework, given two neighbouring points $p$ and $q$ of $E$, the pair $\{p, q\}$ corresponds to an edge element $e(p, q)$ separating $p$ and $q$. See Figure 8, and also Figure 9 below for the decomposition of edges into edge elements. In a partial partition $\pi$, the edge element $e(p, q)$ will be of one of the following four types:

- background: $p$ and $q$ belong both to the background of $\pi$;

- outer: one of $p$ and $q$ belongs to the background of $\pi$, the other to a block of $\pi$;

- separating: $p$ and $q$ belong to two distinct blocks of $\pi$

- inner: $p$ and $q$ belong to the same block of $\pi$.

See Figure 12 of [15]. Only outer and separating edge elements correspond to block boundaries. For partial partitions with the standard order, these 4 types of edge elements are ordered

$$
\text { background }<\text { outer }<\text { separating }<\text { inner },
$$

so that as one climbs from $\pi_{0}$ to $\pi_{n}$, the type of the edge element $e(p, q)$ can only increase. Here we put "outer" and "separating" in bold type, in order to mean that they are "visible" edge elements corresponding to block boundaries, while "background" and "inner" are "invisible".

In the case of a hierarchy of partitions (with the refinement order), since the background is empty, there are only separating and inner edge elements. Thus block boundaries are made of separating edge elements, and as one climbs the hierarchy, they can only disappear by having their edge elements becoming inner. In other words, the above inequality is simplified into

$$
\text { separating }<\text { inner . }
$$

We can partition the set of separating edge elements by grouping together all those that separate the same set of blocks of the partial partition. Thus to every partition of $E$ corresponds a partial partition of edge elements, where each block represents the common boundary of a set of adjacent blocks of the partition of $E$.

We illustrate this in Figure 9 in the framework of images on a cellular complex: each pixel is a 2-cell, and the edge elements are the 0 - and 1-cells, corresponding to corners and side boundaries. As one climbs in the hierarchy, the various boundaries will merge (because they separate the same blocks) or disappear (since they are constituted of inner edges). Thus the partial partition of edge elements will grow according to the regional order.

Let us now consider a hierarchy of partial partitions for the regional order, in other words a chain

$$
\mathbf{0}_{E}=\pi_{0} \stackrel{\mathrm{rg}}{\leq} \ldots \stackrel{\mathrm{rg}}{\leq} \pi_{n}=\varnothing
$$

in $\Pi^{*}(E)$, and the evolution of the corresponding edges; by the above isomorphism, such a chain corresponds to a hierarchy

$$
\mathbf{0}_{\widehat{E}}=\widehat{\mathbf{0}_{E}}=\widehat{\pi_{0}} \leq \ldots \leq \widehat{\pi_{n}}=\widehat{\varnothing}=\mathbf{1}_{\widehat{E}}
$$

in $\Pi(\widehat{E})$ for the refinement order.

Since the background in $\Pi^{*}(E)$ becomes a block in $\Pi(\widehat{E})$, whose role in the order is the same as other blocks, in some sense we do not differentiate between blocks and background. Thus the outer and separating types of edge elements belong together to the category of "visible" edge elements that lie along boundaries between blocks of a partition of $\widehat{E}$; similarly the background and inner types of edge elements belong together to the category of "invisible" edge elements that lie inside a block of a partition of $\widehat{E}$. The two categories are ordered

$$
\text { visible }<\text { invisible },
$$



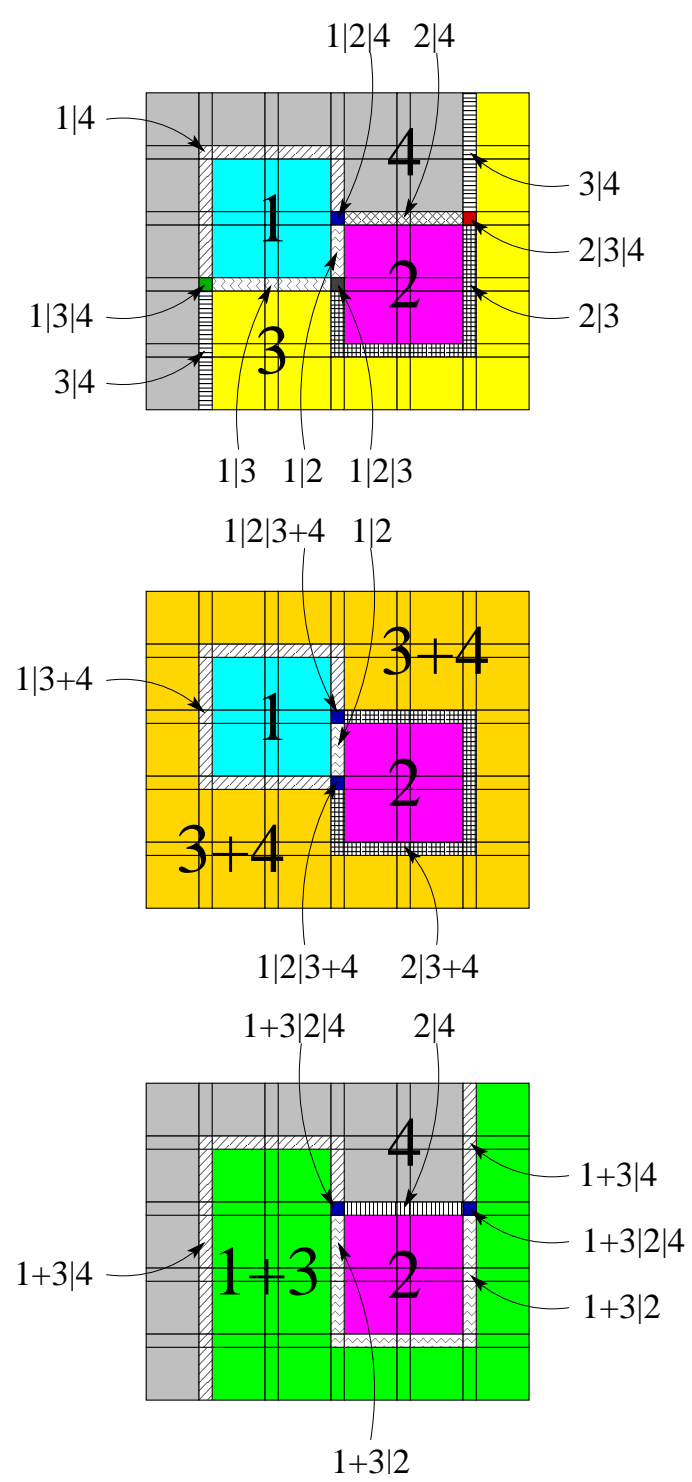

Fig. 9 Top: The 4 blocks (in light colour) are labelled $1,2,3,4$. The boundary between blocks $a_{1}, \ldots, a_{n}(n=2,3)$ is labelled $a_{1}|\cdots| a_{n}$; boundaries between two (resp., three) blocks are shown hatched (resp., with a dark colour). Middle: The blocks 3 and 4 are merged into a block labelled $3+4$, the initial boundaries $a_{1}|\ldots| a_{n}$ are either merged, or removed; note that the boudary $1|2| 3+4$ is disconnected. Bottom: Merging the blocks 1 and 3 into a block labelled $1+3$, the initial boundaries are merged or removed; note that the boundaries $1+3 \mid 4$ and $1+3|2| 4$ are disconnected.

since when climbing in a hierarchy, an edge element can only change from visible to invisible.

On the other hand we distinguish blocks from the background in $\Pi^{*}(E)$, in fact this means that in $\widehat{E}$ we single out the point $\wp$. Thus if for a partition of $\widehat{E}$ a point belongs to the same block as $\wp$, this will remain the case for all partitions higher up in the hierarchy. Therefore the four edge types are ordered along two axes, according to invisibility and to inclusion in the
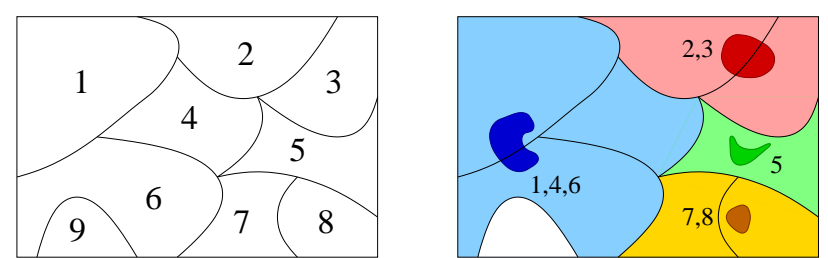

Fig. 10 Left: A territory is partitioned into 9 districts labeled 1 to 9 . Right: A partial partition of the territory made of 4 public service units (in dark colours); the districts served by each unit are filled with the corresponding light colour, and their labels are listed next to the unit, separated by commas. Each unit is included in the union of districts that it serves. Note that district 9 is not served by any unit.

background:

$\begin{array}{ccc}\text { outer } & < & \text { background } \\ \vee & & \vee \\ \text { separating } & < & \text { inner }\end{array}$

As one climbs in the hierarchy, the type of an edge element $e(p, q)$ can only increase.

\section{The linking order}

We will consider a situation where the blocks of a partial partition are regrouped into clusters, then in each cluster a smaller "representative" block is built by taking some points. In other words blocks are merged, then deflated.

We illustrate this through an example. Suppose that a territory is divided into several districts. One wishes to place public service units (for example, schools or postal delivery services); each unit serves one or several districts, and each district is served by at most one unit; it is possible for a district to have no unit (say, a military base or an airport does not need a school). Now for practical reasons the space occupied by each unit will be within the union of districts that it serves; in other words, a unit cannot overlap a district that it does not serve. See Figure 10. The function associating to a district the unit serving it links the initial partition of districts to the partial partition of units.

We will formalize such a construction in three ways. Contrarily to all orders that we have considered up to now, we will not rely on the inclusion relation between individual blocks of two partial partitions, but rather on the overlap between these blocks.

\subsection{Three views of linking}

Given two partial partitions $\pi_{1}$ and $\pi_{2}$, let $\pi \subseteq \pi_{1}$ and consider a map $\lambda: \pi \rightarrow \pi_{2}$ such that for every $C \in \pi_{2}$, 
$C \subseteq \bigcup\{A \in \pi \mid \lambda(A)=C\}$, then we say that $\lambda$ links $\pi_{1}$ to $\pi_{2}$; indeed, each block $C \in \pi_{2}$ links together into a cluster all blocks of $A \in \pi$ such that $\lambda(A)=C$. When $\pi=\pi_{1}$, that is, $\lambda$ is a map $\pi_{1} \rightarrow \pi_{2}$, then we say that $\lambda$ completely links $\pi_{1}$ to $\pi_{2}$.

Lemma 6 Let $\pi \subseteq \pi_{1}$ and $\lambda: \pi \rightarrow \pi_{2}$ that links $\pi_{1}$ to $\pi_{2}$. For any $B \in \pi_{1}$ and $C \in \pi_{2}$, if $B \gamma C$, then $B \in \pi$ and $C=\lambda(B)$.

Proof As $B \gamma C$, there is $p \in B \cap C$, and as $C \subseteq \bigcup\{A \in$ $\pi \mid \lambda(A)=C\}$, there is some $A \in \pi$ such that $\lambda(A)=C$ and $p \in A$, thus the two block $A, B \in \pi_{1}$ overlap, hence $B=A$, so $B \in \pi$ and $\lambda(B)=C$.

This means that a block of $\pi_{1}$ overlaps at most one block of $\pi_{2}$. We introduce thus the following binary relation:

- single overlap: we write $\pi_{1} \vdash \pi_{2}$ or $\pi_{2} \dashv \pi_{1}$ if every block of $\pi_{1}$ overlaps at most one block of $\pi_{2}$,

$\forall B \in \pi_{1}, \forall C, D \in \pi_{2}$,

$[B \gamma C \& B \gamma D] \Longrightarrow C=D$.

It has the following characterization:

Proposition 7 For any $\pi_{1}, \pi_{2} \in \Pi^{*}(E)$, $\pi_{1} \vdash \pi_{2} \Longleftrightarrow \pi_{1} \wedge \mathbf{1}_{\operatorname{supp}\left(\pi_{2}\right)} \leq \pi_{2} \Longleftrightarrow \pi_{1} \wedge \pi_{2} \Leftarrow \pi_{1}$.

In particular,

$$
\pi_{1} \leq \pi_{2} \quad \Longrightarrow \quad \pi_{1} \vdash \pi_{2} \Longrightarrow \pi_{2} \Leftarrow \pi_{1} .
$$

Proof The blocks of $\pi_{1} \wedge \mathbf{1}_{\operatorname{supp}\left(\pi_{2}\right)}$ are all non-empty $B \cap \operatorname{supp}\left(\pi_{2}\right)$ for $B \in \pi_{1}$; here $B \cap \operatorname{supp}\left(\pi_{2}\right)$ is the union of all $B \cap C$ for $C \in \pi_{2}$. If some $B \in \pi_{1}$ overlaps at least two blocks $C_{1}, C_{2} \in \pi_{2}$, then $B \cap \operatorname{supp}\left(\pi_{2}\right)$ overlaps both $C_{1}, C_{2}$, so it cannot be included in one block of $\pi_{2}$, hence $\pi_{1} \wedge \mathbf{1}_{\text {supp }\left(\pi_{2}\right)} \not \leq \pi_{2}$. If each $B \in \pi_{1}$ overlaps at most one block of $\pi_{2}$, then $B \cap \operatorname{supp}\left(\pi_{2}\right)$ either is empty and then is not a block of $\pi_{1} \wedge \mathbf{1}_{\operatorname{supp}\left(\pi_{2}\right)}$, or overlaps exactly one block $C$ of $\pi_{2}$, and then $B \cap \operatorname{supp}\left(\pi_{2}\right)=B \cap C \subseteq C$; hence every block of $\pi_{1} \wedge \mathbf{1}_{\text {supp }\left(\pi_{2}\right)}$ is included in a block of $\pi_{2}$, and $\pi_{1} \wedge \mathbf{1}_{\operatorname{supp}\left(\pi_{2}\right)} \leq \pi_{2}$. Therefore $\pi_{1} \vdash \pi_{2} \Leftrightarrow$ $\pi_{1} \wedge \mathbf{1}_{\text {supp }\left(\pi_{2}\right)} \leq \pi_{2}$.

The blocks of $\pi_{1} \wedge \pi_{2}$ are all non-void $B \cap C$ for $B \in \pi_{1}$ and $C \in \pi_{2}$. If some $B \in \pi_{1}$ overlaps at least two blocks $C_{1}, C_{2} \in \pi_{2}$, then $B \cap C_{1}, B \cap C_{2} \in \pi_{1} \wedge \pi_{2}$, so $B$ contains at least two blocks of $\pi_{1} \wedge \pi_{2}$. If each $B \in \pi_{1}$ overlaps at most one block of $\pi_{2}$, there is at most one $C \in \pi_{2}$ with a non-void $B \cap C$, so $B$ contains at most one block of $\pi_{1} \wedge \pi_{2}$. Therefore $\pi_{1} \vdash \pi_{2} \Leftrightarrow \pi_{1} \wedge \pi_{2} \Leftarrow \pi_{1}$.

If $\pi_{1} \leq \pi_{2}$, then $\operatorname{supp}\left(\pi_{1}\right) \subseteq \operatorname{supp}\left(\pi_{2}\right)$, so $\pi_{1} \leq$ $\mathbf{1}_{\text {supp }\left(\pi_{2}\right)}$ and $\pi_{1} \wedge \mathbf{1}_{\operatorname{supp}\left(\pi_{2}\right)}=\pi_{1} \leq \pi_{2}$, thus $\pi_{1} \vdash \pi_{2}$. Now $\pi_{1} \vdash \pi_{2}$ is equivalent to $\pi_{1} \wedge \pi_{2} \Leftarrow \pi_{1}$, and by (1), the latter implies that $\pi_{2} \Leftarrow \pi_{1}$.
Note that when $\pi_{1} \geq \pi_{2}$, a block of $\pi_{1}$ overlaps a block of $\pi_{2}$ iff it contains it, thus here $\pi_{1} \vdash \pi_{2} \Longleftrightarrow$ $\pi_{2} \Leftarrow \pi_{1}$. Further properties of single overlap are given in Appendix A.

We define the linking order $\stackrel{\text { In }}{\leq}$ as the intersection of the support containment and single overlap relations, $\forall \pi_{1}, \pi_{2} \in \Pi^{*}(E)$ :

$\pi_{1} \stackrel{\ln }{\leq} \pi_{2} \Longleftrightarrow\left[\pi_{1} \vdash \pi_{2} \& \operatorname{supp}\left(\pi_{1}\right) \supseteq \operatorname{supp}\left(\pi_{2}\right)\right]$

In other words, every block of $\pi_{1}$ overlaps at most one block of $\pi_{2}$ and every block of $\pi_{2}$ is included in the union of all blocks of $\pi_{1}$ that overlap it. This relation corresponds to a map that links the two partial partitions, and also to merging then deflating blocks:

Proposition 8 For any $\pi_{1}, \pi_{2} \in \Pi^{*}(E), \pi_{1} \stackrel{\ln }{\leq} \pi_{2}$ if and only if there exists a map that links $\pi_{1}$ to $\pi_{2}$. Moreover, when $\pi_{2} \neq \varnothing$, the following four statements are equivalent:

1. $\pi_{1} \stackrel{\ln }{\leq} \pi_{2}$;

2. there is a map that links $\pi_{1}$ to $\pi_{2}$;

3. there is a map that completely links $\pi_{1}$ to $\pi_{2}$;

4. there exists $\pi_{0} \in \Pi^{*}(E) \backslash\{\varnothing\}$ such that $\pi_{1} \stackrel{\mathrm{m}}{\leq} \pi_{0} \stackrel{\mathrm{i}}{\geq}$ $\pi_{2}$.

Then we necessarily have $\pi_{1} \neq \varnothing$.

Proof Suppose first that $\pi_{1} \stackrel{\ln }{\leq} \pi_{2}$. Let $\pi$ be the set of blocks of $\pi_{1}$ that overlap a block of $\pi_{2}$ :

$$
\pi=\left\{A \in \pi_{1} \mid \exists C \in \pi_{2}, A \gamma C\right\} .
$$

As $\pi_{1} \vdash \pi_{2}$, for $A \in \pi$ there is a unique $C \in \pi_{2}$ with $A \gamma C$, so we define $\lambda: \pi \rightarrow \pi_{2}$ by $\lambda(A)=C$ when $A \gamma C$. Since $\operatorname{supp}\left(\pi_{2}\right) \subseteq \operatorname{supp}\left(\pi_{1}\right)$, each block of $\pi_{2}$ is contained in the union of all blocks of $\pi_{1}$ that overlap it, thus for $C \in \pi_{2}$ :

$$
C \subseteq \bigcup\left\{A \in \pi_{1} \mid A \gamma C\right\}=\bigcup\{A \in \pi \mid \lambda(A)=C\},
$$

hence $\lambda$ links $\pi_{1}$ to $\pi_{2}$.

Suppose next that for some $\pi \subseteq \pi_{1}$ there is a map $\lambda: \pi \rightarrow \pi_{2}$ that links $\pi_{1}$ to $\pi_{2}$. As for every $C \in \pi_{2}$,

$$
C \subseteq \bigcup\{A \in \pi \mid \lambda(A)=C\} \subseteq \operatorname{supp}(\pi) \subseteq \operatorname{supp}\left(\pi_{1}\right),
$$

we have $\operatorname{supp}\left(\pi_{2}\right) \subseteq \operatorname{supp}\left(\pi_{1}\right)$. For any $B \in \pi_{1}$, by Lemma 6 , the only block of $\pi_{2}$ that can overlap $B$ is $\lambda(B)$ for $B \in \pi$. It follows that $\pi_{1} \vdash \pi_{2}$. Therefore $\pi_{1} \stackrel{\text { In }}{\leq} \pi_{2}$. So we have shown that $\pi_{1} \stackrel{\text { In }}{\leq} \pi_{2}$ if and only if there exists a map that links $\pi_{1}$ to $\pi_{2}$. 
Now take $\pi_{2} \neq \varnothing$. Then we cannot have $\operatorname{supp}\left(\pi_{2}\right) \subseteq$ $\operatorname{supp}(\varnothing)$, nor a map that links $\varnothing$ to $\pi_{2}$, nor $\varnothing \stackrel{\mathrm{m}}{\leq} \pi_{0}$ for $\pi_{0} \neq \varnothing$, thus in any case we must have $\pi_{1} \neq \varnothing$.

From the above argument, item 1 implies item 2 and item 3 implies item 1 . Next we show that item 2 implies item 3. Let $\pi \subseteq \pi_{1}$ and $\lambda: \pi \rightarrow \pi_{2}$ that links $\pi_{1}$ to $\pi_{2}$. Define $\lambda^{*}: \pi_{1} \rightarrow \pi_{2}$ as follows: for $A \in \pi$, set $\lambda^{*}(A)=$ $\lambda(A)$; choose a fixed $H \in \pi_{2}$, and for $B \in \pi_{1} \backslash \pi$, set $\lambda^{*}(B)=H$. Then for every $C \in \pi_{2}$,

$$
\begin{gathered}
C \subseteq \bigcup\{A \in \pi \mid \lambda(A)=C\}= \\
\bigcup\left\{A \in \pi \mid \lambda^{*}(A)=C\right\} \subseteq \bigcup\left\{B \in \pi_{1} \mid \lambda^{*}(B)=C\right\},
\end{gathered}
$$

hence $\lambda^{*}$ completely links $\pi_{1}$ to $\pi_{2}$.

There remains to show that items 3 and 4 are equivalent. Suppose item 3: $\lambda$ completely links $\pi_{1}$ to $\pi_{2}$. For any $C \in \pi_{2}$, let $\varphi(C)=\bigcup\left\{B \in \pi_{1} \mid \lambda(B)=C\right\}$ and let $\pi_{0}=\left\{\varphi(C) \mid C \in \pi_{2}\right\}$; as $\lambda$ is a map, for any two distinct $C, C^{\prime} \in \pi_{2}, \varphi(C)$ and $\varphi\left(C^{\prime}\right)$ may not contain a common block of $\pi_{1}$, so they are disjoint; thus $\pi_{0}$ is a non-void partial partition. For any $B \in \pi_{1}$, $B \subseteq \varphi(\lambda(B)) \in \pi_{0}$, so $\pi_{1} \leq \pi_{0}$, and for any $C \in \pi_{2}$, $\varphi(C) \subseteq \operatorname{supp}\left(\pi_{1}\right)$, so $\operatorname{supp}\left(\pi_{0}\right) \subseteq \operatorname{supp}\left(\pi_{1}\right)$; we deduce that $\pi_{1} \stackrel{\mathrm{m}}{\leq} \pi_{0}$. By definition of linking, for any $C \in \pi_{2}$, $C \subseteq \varphi(C)$; now for any other $C^{\prime} \in \pi_{2}, C^{\prime} \subseteq \varphi\left(C^{\prime}\right)$ and $\varphi\left(C^{\prime}\right)$ is disjoint from $\varphi(C)$, so $C^{\prime} \nsubseteq \varphi(C)$; thus $\varphi$ is a bijection $\pi_{2} \rightarrow \pi_{0}$, and we deduce that $\pi_{0} \geq \pi_{2}$. Therefore item 4 holds.

Conversely, suppose item 4: there is $\pi_{0} \in \Pi^{*}(E) \backslash$ $\{\varnothing\}$ such that $\pi_{1} \stackrel{\mathrm{m}}{\leq} \pi_{0} \stackrel{\mathrm{i}}{\geq} \pi_{2}$. For any $A \in \pi_{1}$, there is a unique $B \in \pi_{0}$ such that $A \subseteq B$, and there is a unique $C \in \pi_{2}$ such that $B \supseteq C$; we define thus $\lambda(A)=C$, hence $\lambda$ is a map $\pi_{1} \rightarrow \pi_{2}$. For any $C \in \pi_{2}$, there is a unique $B \in \pi_{0}$ such that $B \supseteq C$, and $B$ is a union of blocks of $\pi_{1}$; now for each $A \in \pi_{1}$ such that $A \subseteq B$, we have $\lambda(A)=C$, hence

$$
\begin{gathered}
C \subseteq B=\bigcup\left\{A \in \pi_{1} \mid A \subseteq B\right\} \\
\subseteq \bigcup\left\{A \in \pi_{1} \mid \lambda(A)=C\right\}
\end{gathered}
$$

which means that $\lambda$ completely links $\pi_{1}$ to $\pi_{2}$, that is, item 3 .

We illustrated in Figure 3 the equivalence between items 1 and 4, namely that for $\pi_{1}, \pi_{2} \in \Pi^{*}(E) \backslash\{\varnothing\}$,

$$
\pi_{1} \stackrel{\ln }{\leq} \pi_{2} \Longleftrightarrow \exists \pi_{0} \in \Pi^{*}(E) \backslash\{\varnothing\}, \pi_{1} \stackrel{\mathrm{m}}{\leq} \pi_{0} \stackrel{\mathrm{i}}{\geq} \pi_{2},
$$

in other words, for non-void partial partitions, linking is generated by merging followed by inverse inflating. The equivalence with item 2 appears implicitly, as $\pi$ consists of all hatched blocks of $\pi_{1}$, and $\lambda$ associates to each block of $\pi$ the unique block of $\pi_{2}$ with the same hatching.

When $\pi_{2}=\varnothing$, we have trivially $\pi_{1} \stackrel{\ln }{\leq} \varnothing$ and for $\pi=\varnothing$ the empty map $\varnothing \rightarrow \varnothing$ links $\pi_{1}$ to $\varnothing$; thus items 1 and 2 hold. However if $\pi_{1} \neq \varnothing$, there is no map that completely links $\pi_{1}$ to $\varnothing$, and for $\pi_{1} \stackrel{m}{\leq} \pi_{0}$ we have $\pi_{0} \neq \varnothing$, so $\pi_{0} \stackrel{i}{\geq} \pi_{2}$; thus items 3 and 4 cannot hold in this case.

Hence in the analysis and characterization of the linking order, we have to distinguish $\varnothing$ from non-void partitions.

\subsection{Characterization of the linking order}

Theorem 9 The linking order $\stackrel{\ln }{\leq}$ is a partial order relation on $\Pi^{*}(E)$. It contains the inverse inclusion-inflating $\stackrel{\mathrm{i}}{\supseteq}$ and regional $\stackrel{\mathrm{rg}}{\leq}$ orders, that is, for any $\pi_{1}, \pi_{2} \in$ $\Pi^{*}(E)$, each of $\pi_{1} \supseteq \pi_{2}$ and $\pi_{1} \stackrel{\text { rg }}{\leq} \pi_{2}$ implies $\pi_{1} \stackrel{\ln }{\leq} \pi_{2}$. The greatest and least elements are $\varnothing$ and $\mathbf{0}_{E}$. For any $\pi_{1}, \pi_{2} \in \Pi^{*}(E)$ we have

$\pi_{1} \stackrel{\mathrm{m}}{\leq} \pi_{2} \Longleftrightarrow\left[\pi_{1} \stackrel{\ln }{\leq} \pi_{2} \& \operatorname{supp}\left(\pi_{1}\right)=\operatorname{supp}\left(\pi_{2}\right)\right]$.

For non-void partial partitions (in $\left.\Pi^{*}(E) \backslash\{\varnothing\}\right)$, the maximal elements are the $\mathbf{1}_{\{p\}}$ for $p \in E$ and the covering relation is $\stackrel{\mathrm{m}}{\prec} \cup \stackrel{\mathrm{i}}{\succ}$. On the other hand, $\varnothing$ covers an element of $\Pi^{*}(E) \backslash\{\varnothing\}$ iff it is maximal, i.e., it is $\mathbf{1}_{\{p\}}$ for some $p \in E$, and then $\mathbf{1}_{\{p\}} \succ^{\llcorner} \varnothing$.

Let $E$ be finite. Then $\Pi^{*}(E) \backslash\{\varnothing\}$ ordered by $\leq$ in graded by $\left(h_{r}, h_{b}\right)$, equivalently by $\left(-h_{c},-h_{s}\right)$, for $(\stackrel{\mathrm{m}}{\prec}$ , $\stackrel{\mathrm{i}}{\succ})$, that is, for any $\pi_{1}, \pi_{2} \in \Pi^{*}(E) \backslash\{\varnothing\}$ we have

$$
\begin{aligned}
& \pi_{1} \stackrel{\ln }{<} \pi_{2} \Longrightarrow {\left[\begin{array}{l}
h_{s}\left(\pi_{1}\right) \geq h_{s}\left(\pi_{2}\right) \& \\
h_{c}\left(\pi_{1}\right) \geq h_{c}\left(\pi_{2}\right) \& \\
\left(h_{s}+h_{c}\right)\left(\pi_{1}\right)>\left(h_{s}+h_{c}\right)\left(\pi_{2}\right)
\end{array}\right], } \\
& \pi_{1} \stackrel{\mathrm{m}}{\prec} \pi_{2} \Longrightarrow\left[\begin{array}{l}
h_{s}\left(\pi_{2}\right)=h_{s}\left(\pi_{1}\right) \& \\
h_{c}\left(\pi_{2}\right)=h_{c}\left(\pi_{1}\right)-1
\end{array}\right], \\
& \pi_{1} \stackrel{\mathrm{i}}{\succ} \pi_{2} \Longrightarrow\left[\begin{array}{l}
h_{c}\left(\pi_{2}\right)=h_{c}\left(\pi_{1}\right) \& \\
h_{s}\left(\pi_{2}\right)=h_{s}\left(\pi_{1}\right)-1
\end{array}\right] .
\end{aligned}
$$

For $\pi \in \Pi^{*}(E) \backslash\{\varnothing\}$, the height of $\pi$ is $h_{r}(\pi)+h_{b}(\pi)$. On the other hand, the height of $\varnothing$ is $2|E|-1=h_{r}(\varnothing)+$ $h_{b}(\varnothing)-1$.

Proof By (8) and Proposition 7, for $\pi_{1}, \pi_{2} \in \Pi^{*}(E)$ we have $\pi_{1} \stackrel{\text { In }}{\leq} \pi_{2}$ iff $\pi_{1} \wedge \mathbf{1}_{\operatorname{supp}\left(\pi_{2}\right)} \leq \pi_{2} \& \operatorname{supp}\left(\pi_{1}\right) \supseteq$ $\operatorname{supp}\left(\pi_{2}\right)$. Thus, under the fixed condition $\operatorname{supp}\left(\pi_{1}\right)=$ 
$\operatorname{supp}\left(\pi_{2}\right)$, we have $\pi_{1} \leq \pi_{2}$ iff $\pi_{1} \wedge \mathbf{1}_{\text {supp }\left(\pi_{2}\right)} \leq \pi_{2}$, that is, $\pi_{1} \wedge \mathbf{1}_{\text {supp }\left(\pi_{1}\right)} \leq \pi_{2}$, in other words $\pi_{1} \leq \pi_{2}$. Therefore

$$
\begin{aligned}
& {\left[\pi_{1} \stackrel{\ln }{\leq} \pi_{2} \& \operatorname{supp}\left(\pi_{1}\right)=\operatorname{supp}\left(\pi_{2}\right)\right] } \\
\Longleftrightarrow & {\left[\pi_{1} \leq \pi_{2} \& \operatorname{supp}\left(\pi_{1}\right)=\operatorname{supp}\left(\pi_{2}\right)\right] } \\
\Longleftrightarrow & \pi_{1} \leq \pi_{2},
\end{aligned}
$$

so (9) holds.

Both support containment and single overlap are reflexive relations, so their intersection is reflexive. Let us show that this intersection is anti-symmetric. If $\pi_{1} \stackrel{\ln }{\leq} \pi_{2}$ and $\pi_{2} \leq \pi_{1}$, then $\operatorname{supp}\left(\pi_{1}\right) \supseteq \operatorname{supp}\left(\pi_{2}\right)$ and $\operatorname{supp}\left(\pi_{2}\right) \supseteq$ $\operatorname{supp}\left(\pi_{1}\right)$, that is, $\operatorname{supp}\left(\pi_{1}\right)=\operatorname{supp}\left(\pi_{2}\right)$; then (9) gives $\pi_{1} \stackrel{\mathrm{m}}{\leq} \pi_{2}$ and $\pi_{2} \stackrel{\mathrm{m}}{\leq} \pi_{1}$, hence $\pi_{1}=\pi_{2}$. Let us show that it is transitive. Let $\pi_{0} \stackrel{\ln }{\leq} \pi_{1} \stackrel{\ln }{\leq} \pi_{2}$, that is, $\pi_{0} \vdash \pi_{1} \vdash \pi_{2}$ and $\operatorname{supp}\left(\pi_{0}\right) \supseteq \operatorname{supp}\left(\pi_{1}\right) \supseteq \operatorname{supp}\left(\pi_{2}\right)$; then $\mathbf{1}_{\operatorname{supp}\left(\pi_{2}\right)} \leq$ $\mathbf{1}_{\text {supp }\left(\pi_{1}\right)}$ and Proposition 7 gives $\pi_{0} \wedge \mathbf{1}_{\text {supp }\left(\pi_{1}\right)} \leq \pi_{1}$ and $\pi_{1} \wedge \mathbf{1}_{\text {supp }\left(\pi_{2}\right)} \leq \pi_{2}$, hence

$$
\begin{gathered}
\pi_{0} \wedge \mathbf{1}_{\operatorname{supp}\left(\pi_{2}\right)}=\pi_{0} \wedge\left(\mathbf{1}_{\operatorname{supp}\left(\pi_{1}\right)} \wedge \mathbf{1}_{\operatorname{supp}\left(\pi_{2}\right)}\right) \\
=\left(\pi_{0} \wedge \mathbf{1}_{\operatorname{supp}\left(\pi_{1}\right)}\right) \wedge \mathbf{1}_{\operatorname{supp}\left(\pi_{2}\right)} \leq \pi_{1} \wedge \mathbf{1}_{\operatorname{supp}\left(\pi_{2}\right)} \leq \pi_{2},
\end{gathered}
$$

thus $\pi_{0} \vdash \pi_{2}$; also $\operatorname{supp}\left(\pi_{0}\right) \supseteq \operatorname{supp}\left(\pi_{2}\right)$, hence $\pi_{0} \stackrel{\ln }{\leq} \pi_{2}$. Therefore $\leq$ in a partial order.

If $\pi_{1} \supseteq \pi_{2}$, then (2) gives $\pi_{1} \wedge \mathbf{1}_{\operatorname{supp}\left(\pi_{2}\right)}=\pi_{2}$; thus $\pi_{1} \wedge \mathbf{1}_{\text {supp }\left(\pi_{2}\right)} \leq \pi_{2}$, so $\pi_{1} \vdash \pi_{2}$ by Proposition 7 , also $\pi_{2}=\pi_{1} \wedge \mathbf{1}_{\text {supp }\left(\pi_{2}\right)} \leq \pi_{1}$, so $\operatorname{supp}\left(\pi_{2}\right) \subseteq \operatorname{supp}\left(\pi_{1}\right)$, hence $\pi_{1} \stackrel{\ln }{\leq} \pi_{2}$. If $\pi_{1} \stackrel{\text { rg }}{\leq} \pi_{2}$, by Proposition $4, \operatorname{supp}\left(\pi_{1}\right) \supseteq$ $\operatorname{supp}\left(\pi_{2}\right)$ and $\pi_{1} \leq \pi_{2} \cup\left\{\operatorname{back}\left(\pi_{2}\right)\right\}$; restricting to the support of $\pi_{2}$ we get:

$$
\pi_{1} \wedge \mathbf{1}_{\text {supp }\left(\pi_{2}\right)} \leq\left(\pi_{2} \cup\left\{\operatorname{back}\left(\pi_{2}\right)\right\}\right) \wedge \mathbf{1}_{\text {supp }\left(\pi_{2}\right)}=\pi_{2},
$$

so Proposition 7 gives $\pi_{1} \vdash \pi_{2}$, hence $\pi_{1} \stackrel{\ln }{\leq} \pi_{2}$. Therefore $\stackrel{\text { In }}{\leq}$ contains $\stackrel{\mathrm{i}}{\supseteq}$ and $\stackrel{\text { rg }}{\leq}$.

By Theorem $5, \mathbf{0}_{E}$ and $\varnothing$ are the least and greatest elements of $\Pi^{*}(E)$ for the regional order, so for any $\pi \in \Pi^{*}(E), \mathbf{0}_{E} \underset{\ln }{\stackrel{\mathrm{rg}}{\leq}} \pi \underset{\ln }{\mathrm{rg}} \boldsymbol{\varnothing}$, and as $\stackrel{\ln }{\leq}$ contains $\stackrel{\text { rg }}{\leq}$, we deduce that $\mathbf{0}_{E} \stackrel{\ln }{\leq} \pi \stackrel{\ln }{\leq} \varnothing$, that is, $\mathbf{0}_{E}$ and $\varnothing$ are the least and greatest elements of $\Pi^{*}(E)$ for the linking order. For $\pi \in \Pi^{*}(E) \backslash\{\varnothing\}$, if $\pi$ has several blocks or has a block with several points, then a larger element of $\Pi^{*}(E) \backslash\{\varnothing\}$ is obtained by merging blocks or deflating a block respectively; thus to be maximal, it must have a single block with a single point, i.e., $\pi=\mathbf{1}_{\{p\}}$ for some $p \in E$; conversely, in $\mathbf{1}_{\{p\}}$, no block merging or deflation is possible, so it is maximal in $\Pi^{*}(E) \backslash\{\varnothing\}$.

Let $\stackrel{\ln }{\prec}$ be the covering relation for $\stackrel{\ln }{\leq}$. The hypothesis of Lemmas 1 and 2 is satisfied with $P=\Pi^{*}(E) \backslash\{\varnothing\}$ with the partial order $\leq$ (for $\leq$ ), which is the transitive closure of the union of the two partial orders $\stackrel{m}{\leq}^{m}$ and $\stackrel{i}{\geq}$ (standing for $\stackrel{\alpha}{\leq}$ and $\stackrel{\beta}{\leq}$ ), $Q=\mathcal{P}(E)$ with the partial order $\supseteq$ for $\sqsubseteq$, and the support supp for the map $f$. Since the covering relations of $\stackrel{\mathrm{m}}{\leq}$ and $\stackrel{i}{\geq}$ are $\stackrel{\mathrm{m}}{\prec}$ and $\succ^{\mathrm{i}}$ respectively, we deduce (from Lemmas 1 and 2) that for any $\pi_{1}, \pi_{2} \in \Pi^{*}(E) \backslash\{\varnothing\}$ : (i) if $\pi_{1} \stackrel{\ln }{\prec} \pi_{2}$, then $\pi_{1} \stackrel{\mathrm{m}}{\prec} \pi_{2}$ or $\pi_{1} \stackrel{\mathrm{i}}{\succ} \pi_{2}$; (ii) if $\pi_{1} \stackrel{\mathrm{m}}{\prec} \pi_{2}$, then $\pi_{1} \stackrel{\ln }{\prec} \pi_{2}$. Let us now show that (iii) if $\pi_{1} \stackrel{i}{\succ} \pi_{2}$, then $\pi_{1} \stackrel{\ln }{\prec} \pi_{2}$. Let $\pi_{1} \stackrel{\mathrm{i}}{\succ} \pi_{2}$, where $\pi_{2}$ is obtained by removing a point $p$ from a block of $\pi_{1}$ that also contains another point; then $\pi_{2}=\left\{C \backslash\{p\} \mid C \in \pi_{1}\right\}$ (where each such $C \backslash\{p\} \neq \emptyset$ ). By Proposition 8 , a chain $\pi_{1} \stackrel{\ln }{\leq} \ldots \stackrel{\ln }{\leq} \pi_{2}$ in $\Pi^{*}(E) \backslash\{\emptyset\}$ alternates merging $\stackrel{m}{\leq}$ and inverse inflation $\stackrel{i}{\geq}$, with the support monotonously decreasing; since globally there is only one decrease of the support, where the point $p$ is removed from a block, this chain reduces to $\pi_{1} \stackrel{\mathrm{m}}{\leq} \pi_{3} \stackrel{\mathrm{i}}{>}$ $\pi_{4} \stackrel{\mathrm{m}}{\leq} \pi_{2}$, where $\pi_{4}$ is obtained by removing the point $p$ from a block of $\pi_{3}$, thus $\pi_{4}=\left\{C \backslash\{p\} \mid C \in \pi_{3}\right\}$. Then for any $q \in \operatorname{supp}\left(\pi_{2}\right)$ we have $\mathrm{Cl}_{\pi_{2}}(q)=\mathrm{Cl}_{\pi_{1}}(q) \backslash\{p\}$, $\mathrm{Cl}_{\pi_{1}}(q) \subseteq \mathrm{Cl}_{\pi_{3}}(q), \mathrm{Cl}_{\pi_{4}}(q)=\mathrm{Cl}_{\pi_{3}}(q) \backslash\{p\}$ and $\mathrm{Cl}_{\pi_{4}}(q) \subseteq$ $\mathrm{Cl}_{\pi_{2}}(q)$. We deduce that $\mathrm{Cl}_{\pi_{1}}(q) \backslash\{p\} \subseteq \mathrm{Cl}_{\pi_{3}}(q) \backslash\{p\}=$ $\mathrm{Cl}_{\pi_{4}}(q) \subseteq \mathrm{Cl}_{\pi_{2}}(q)=\mathrm{Cl}_{\pi_{1}}(q) \backslash\{p\}$, so $\mathrm{Cl}_{\pi_{1}}(q) \backslash\{p\}=$ $\mathrm{Cl}_{\pi_{3}}(q) \backslash\{p\}=\mathrm{Cl}_{\pi_{4}}(q)=\mathrm{Cl}_{\pi_{2}}(q)$; as the last equality holds for any $q \in \operatorname{supp}\left(\pi_{2}\right)$, we get $\pi_{4}=\pi_{2}$. Since $\mathrm{Cl}_{\pi_{1}}(q) \subseteq \mathrm{Cl}_{\pi_{3}}(q)$ and $\mathrm{Cl}_{\pi_{1}}(q) \backslash\{p\}=\mathrm{Cl}_{\pi_{3}}(q) \backslash\{p\}$, we get $\mathrm{Cl}_{\pi_{3}}(q)=\mathrm{Cl}_{\pi_{1}}(q)$ or $\mathrm{Cl}_{\pi_{3}}(q)=\mathrm{Cl}_{\pi_{1}}(q) \cup\{p\}$; the second case means that $\mathrm{Cl}_{\pi_{3}}(q)$ is the merging of the block $\mathrm{Cl}_{\pi_{1}}(q)$ with a block reduced to the singleton $\{p\}$, which is impossible; hence $\mathrm{Cl}_{\pi_{3}}(q)=\mathrm{Cl}_{\pi_{1}}(q)$ for any $q \in \operatorname{supp}\left(\pi_{2}\right)$, so $\pi_{3}=\pi_{1}$. Therefore $\pi_{1} \stackrel{\ln }{\prec} \pi_{2}$. We have thus shown that the covering relation for $\leq$ on $\Pi^{*}(E) \backslash\{\varnothing\}$ is $\stackrel{\mathrm{m}}{\prec} \cup \stackrel{\mathrm{i}}{\succ}$.

For $\pi \in \Pi^{*}(E) \backslash\{\varnothing\}$, we have $\pi \stackrel{\ln }{<} \varnothing$, and for $\pi^{\prime} \in$ $\Pi^{*}(E)$ with $\pi \stackrel{\ln }{<} \pi^{\prime}$, we have $\pi^{\prime} \stackrel{\ln }{<} \varnothing$; thus $\varnothing$ does not cover $\pi$ iff there is $\pi^{\prime} \in \Pi^{*}(E)$ such that $\pi \stackrel{\ln }{<} \pi^{\prime}$; hence $\varnothing$ covers $\pi$ iff $\pi$ is a maximal element of $\Pi^{*}(E) \backslash\{\varnothing\}$, that is, $\mathbf{1}_{\{p\}}$ for some $p \in E$, and indeed $\mathbf{1}_{\{p\}} \stackrel{\ln }{\prec} \varnothing$ and $\mathbf{1}_{\{p\}} \stackrel{\mathrm{s}}{\succ} \varnothing$.

Assume that $E$ is finite, and let $\pi_{1}, \pi_{2} \in \Pi^{*}(E) \backslash$ $\{\varnothing\}$ such that $\pi_{1} \stackrel{\ln }{\leq} \pi_{2}$. By Proposition 8, there exists $\pi_{0} \in \Pi^{*}(E) \backslash\{\varnothing\}$ such that $\pi_{1} \leq \pi_{0} \geq \pi_{2}$; then by $[15]$ (see there the analysis of $\stackrel{m}{\leq}$ and $\stackrel{i}{\leq}$ ) we have:

(i) $h_{s}\left(\pi_{1}\right)=h_{s}\left(\pi_{0}\right)$ and $h_{c}\left(\pi_{1}\right) \geq h_{c}\left(\pi_{0}\right)$; for $\pi_{1} \stackrel{\mathrm{m}}{<} \pi_{0}$ we have $h_{c}\left(\pi_{1}\right)>h_{c}\left(\pi_{0}\right)$. 


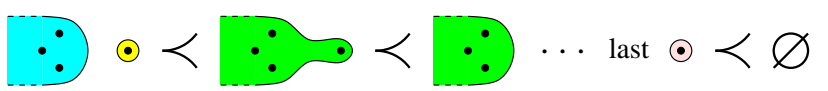

Fig. 11 Removing a singleton block that is not the last block is done in two steps, merging it with another block, then deflating that block. Removing the last block is done in a single step.

(ii) $h_{c}\left(\pi_{0}\right)=h_{c}\left(\pi_{2}\right)$ and $h_{s}\left(\pi_{0}\right) \geq h_{s}\left(\pi_{2}\right)$; for $\pi_{0} \stackrel{\text { i }}{>} \pi_{2}$, $h_{s}\left(\pi_{0}\right)>h_{s}\left(\pi_{2}\right)$.

Thus $h_{s}\left(\pi_{1}\right)=h_{s}\left(\pi_{0}\right) \geq h_{s}\left(\pi_{2}\right)$ and $h_{c}\left(\pi_{1}\right) \geq h_{c}\left(\pi_{0}\right)=$ $h_{c}\left(\pi_{2}\right)$. If $\pi_{1} \stackrel{\ln }{<} \pi_{2}$, then one of the following holds: (a) $\pi_{1} \stackrel{\mathrm{m}}{<} \pi_{0}$, so $h_{c}\left(\pi_{1}\right)>h_{c}\left(\pi_{0}\right)=h_{c}\left(\pi_{2}\right)$; (b) $\pi_{0} \stackrel{\text { i }}{>}$ $\pi_{2}$, so $h_{s}\left(\pi_{1}\right)=h_{s}\left(\pi_{0}\right)>h_{s}\left(\pi_{2}\right)$. Thus for $\pi_{1} \stackrel{\ln }{<} \pi_{2}$, $h_{s}\left(\pi_{1}\right) \geq h_{s}\left(\pi_{2}\right)$ and $h_{c}\left(\pi_{1}\right) \geq h_{c}\left(\pi_{2}\right)$, with at least one inequality being strict, hence $\left(h_{s}+h_{c}\right)\left(\pi_{1}\right)>\left(h_{s}+\right.$ $\left.h_{c}\right)\left(\pi_{2}\right)$. An argument similar to (i) shows that if $\pi_{1} \stackrel{\mathrm{m}}{\prec}$ $\pi_{2}$, then $h_{s}\left(\pi_{2}\right)=h_{s}\left(\pi_{1}\right)$ and $h_{c}\left(\pi_{2}\right)=h_{c}\left(\pi_{1}\right)-1$, and an argument similar to (ii) shows that if $\pi_{1} \stackrel{i}{\succ} \pi_{2}$, then $h_{c}\left(\pi_{2}\right)=h_{c}\left(\pi_{1}\right)$ and $h_{s}\left(\pi_{2}\right)=h_{s}\left(\pi_{1}\right)-1$. Therefore the order $\stackrel{\ln }{\leq}$ on $\Pi^{*}(E) \backslash\{\varnothing\}$ is graded by $\left(-h_{c},-h_{s}\right)$ for $(\stackrel{\mathrm{m}}{\prec}, \stackrel{\mathrm{i}}{\succ})$. As $h_{r}=|E|-h_{c}$ and $h_{b}=|E|-h_{s}$, cf. $(3,4)$, with $|E|$ constant, it is also graded by $\left(h_{r}, h_{b}\right)$.

For $\pi \in \Pi^{*}(E) \backslash\{\varnothing\}$, the length of the interval $\left[\mathbf{0}_{E}, \pi\right]$ is $h_{r}(\pi)+h_{b}(\pi)-h_{r}\left(\mathbf{0}_{E}\right)-h_{b}\left(\mathbf{0}_{E}\right)=h_{r}(\pi)+$ $h_{b}(\pi)-0-0=h_{r}(\pi)+h_{b}(\pi)$; as $\mathbf{0}_{E}$ is the least element, it is thus the height of $\pi$. Now since $\mathbf{1}_{\{p\}} \stackrel{\text { In }}{\prec} \varnothing$ for any $p \in E$, the height of $\varnothing$ is $1+h_{r}\left(\mathbf{1}_{\{p\}}\right)+h_{b}\left(\mathbf{1}_{\{p\}}\right)=$ $1+2(|E|-1)=2|E|-1=h_{r}(\varnothing)+h_{b}(\varnothing)-1$.

We know from [15] that the inverse inclusion-inflating order $\stackrel{i}{\supseteq}$ contains the inverse inflating $\underset{i}{\geq}$ and inverse inclusion $\supseteq$ orders, and from Theorem 5 that the regional order $\stackrel{r g}{\leq}$ contains the merging $\stackrel{m}{\leq}$ and inverse inclusion $\supseteq$ orders; thus the linking order $\leq$ contains also the merging $\stackrel{m}{\leq}$, inverse inclusion $\supseteq$ and inverse inflating $\stackrel{i}{\geq}$ orders.

Note that the change of the height function on the greatest element (the formula is decreased by 1) coincides with the change in the covering relation, from $\stackrel{\mathrm{m}}{\prec} \cup \stackrel{\mathrm{i}}{\succ}$ in $\Pi^{*}(E) \backslash\{\varnothing\}$ to $\stackrel{\mathrm{s}}{\succ}$ between maximal elements of $\Pi^{*}(E) \backslash\{\varnothing\}$ and $\varnothing$. Indeed, removing a singleton block increases both $h_{r}$ and $h_{b}$ by 1 ; now in $\Pi^{*}(E) \backslash\{\varnothing\}$ removing the singleton block requires two steps, merging it with another block then deflating the resulting block $(\stackrel{m}{\prec}$ then $\stackrel{i}{\succ})$, while from a maximal element of $\Pi^{*}(E) \backslash\{\varnothing\}$ to $\varnothing$, the removal of the unique singleton block is done in one step $\stackrel{\text { s }}{\succ}$. See Figure 11 .
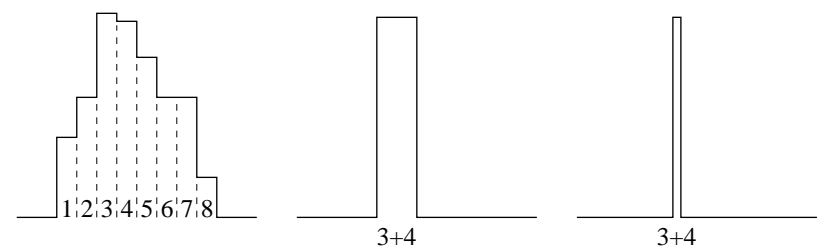

Fig. 12 Left: the flat zones with non-zero grey-level are numbered 1 to 8 . Middle and right: in grey-level thinning, the non-peak zones are removed, and the two peak zones (3 and 4) are merged, then this merged zone is deflated.

\subsection{Possible applications}

The linking order is useful in the analysis of situations where a partial partition can be improved by reducing, removing or merging blocks.

In the Introduction, we discussed skeletonization of binary images: this operation deflates connected components, then cleaning the skeleton to remove spurious branches removes blocks in a partition of the skeleton, also such blocks can be merged in order to simplify the representation.

Similarly, skeletonization (or thinning) of a greylevel image can be seen as an operation on the partition of flat zones (i.e., maximal connected zones of constant grey-level); setting the zones with minimum grey-level as the background, the zones with non-minimum greylevel will form a partial partition. Now skeletonization will thin peak zones while non-peak zones will be removed; furthermore, small variations in peaks can be smoothed out, leading to a merging of the zones on the peaks before thinning the merged zones. See Figure 12 . This thinning can be operated directly at the level of the partial partition, not that of the pixels, and it relies on block removal, block merging and block deflating, thus a growth for the linking order.

In the Introduction, we also considered the processing of region markers in segmentation algorithms based on region growing or watershed, and saw that markers can be:

- reduced in order to maintain each one inside the corresponding region, in other words to avoid spilling into other regions;

- merged in order to avoid oversegmentation;

- removed in order to suppress "parasitic" regions; the latter are then apportioned between neighbouring regions).

In the partial partition of markers, the corresponding operations are deflating, merging and removing blocks, thus the partial partition of markers grows for the linking order. 


\section{Partial apportioning and partial merging}

Block apportioning was proposed in order to eliminate "parasitic" classes in an image segmentation [20,21]; such classes lie along transitions between objects, so they must be removed and their contents must be apportioned between the neighbouring classes corresponding to significant objects. When the segmentation is a partial partition including the objects but not the background, the "parasitic" classes can also lie along the transitions between objects and the background; when removing such classes, their contents must be apportioned between neighbouring classes and the background.

We will thus generalize the operation of block apportioning in order to apportion blocks between other blocks and the background. We will also consider a particular case which generalizes block merging. We will thus obtain two new partial orders on $\Pi^{*}(E)$, the partial apportioning and partial merging orders.

\subsection{The apportioning order}

We recall from [16] the definition and main properties of the apportioning order. Let $\pi_{1}, \pi_{2} \in \Pi^{*}(E) \backslash\{\varnothing\}$ and consider a map $\beta: \pi_{2} \rightarrow \pi_{1}$. Let $\beta\left(\pi_{2}\right)=\{\beta(C) \mid C \in$ $\left.\pi_{2}\right\}$. Then the following two conditions are equivalent:

1. $\operatorname{supp}\left(\pi_{1}\right)=\operatorname{supp}\left(\pi_{2}\right)$ and for all $C \in \pi_{2}, \beta(C) \subseteq C$.

2. There is a map $\alpha: \operatorname{supp}\left(\pi_{1} \backslash \beta\left(\pi_{2}\right)\right) \rightarrow \beta\left(\pi_{2}\right)$ such that for every $C \in \pi_{2}, C=\beta(C) \cup \alpha^{-1}(\beta(C))$.

We say then that $\beta$ apportions $\pi_{1}$ into $\pi_{2}$. Here $\beta$ is an injection $\pi_{2} \rightarrow \pi_{1}$ which selects for every block of $\pi_{2}$ a block of $\pi_{1}$ contained in it. Furthermore, for a given $\beta$, the map $\alpha$ is unique, it is defined by

$\forall p \in \operatorname{supp}\left(\pi_{1} \backslash \beta\left(\pi_{2}\right)\right), \alpha(p)=\beta\left(\mathrm{Cl}_{\pi_{2}}(p)\right)$.

It describes the apportioning of the blocks of $\pi_{1} \backslash \beta\left(\pi_{2}\right)$ between the blocks of $\beta\left(\pi_{2}\right)$, since each $C \in \pi_{2}$ is obtained by adding to $\beta(C)$ all points $p$ in blocks of $\pi_{1} \backslash \beta\left(\pi_{2}\right)$ satisfying $\alpha(p)=\beta(C)$. See Figure 13. Furthermore, we have $\pi_{1}=\pi_{2} \Leftrightarrow \beta\left(\pi_{2}\right)=\pi_{1} \Leftrightarrow \beta\left(\pi_{2}\right)=$ $\pi_{2}$.

We define the apportioning order $\stackrel{\text { a }}{\leq}$ on $\Pi^{*}(E)$ as the intersection of the building order and the support equality relation: $\forall \pi_{1}, \pi_{2} \in \Pi^{*}(E)$,

$\pi_{1} \stackrel{\mathrm{a}}{\leq} \pi_{2} \Longleftrightarrow\left[\pi_{1} \Subset \pi_{2} \& \operatorname{supp}\left(\pi_{1}\right)=\operatorname{supp}\left(\pi_{2}\right)\right]$.

Now given $\pi_{1}, \pi_{2} \in \Pi^{*}(E) \backslash\{\emptyset\}$, we have $\pi_{1} \stackrel{\text { a }}{\leq} \pi_{2}$ if and only if there exists $\beta: \pi_{2} \rightarrow \pi_{1}$ such that $\beta$ apportions $\pi_{1}$ into $\pi_{2}$. Note that the map $\beta$ is not necessarily unique: if a block $C \in \pi_{2}$ contains several blocks

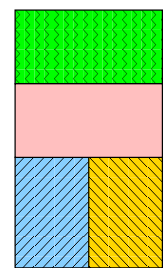

$\pi_{1}$

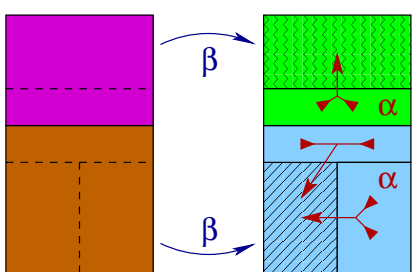

$\pi_{2}$
Fig. 13 Left: $\pi_{1}$; middle: $\pi_{2}$; here $\pi_{1} \stackrel{\text { a }}{\leq} \pi_{2}$, the blocks of $\pi_{1}$ included in a block of $\pi_{2}$ are hatched. Right: one possible choice for $\beta$, and the resulting $\alpha$, in order to apportion $\pi_{1}$ into $\pi_{2}$.

$B_{i} \in \pi_{1}(i \in I)$, then for $\beta(C)$ we can choose any $B_{i}$ for $\beta(C)$. Furthermore,

$$
\pi_{1} \stackrel{\mathrm{a}}{<} \pi_{2} \Longleftrightarrow\left[\begin{array}{c}
\operatorname{supp}\left(\pi_{1}\right)=\operatorname{supp}\left(\pi_{2}\right) \&\left|\pi_{1}\right| \geq 2 \\
\& \exists \pi \subset \pi_{1}, \pi \neq \varnothing, \pi \stackrel{\mathrm{i}}{<} \pi_{2}
\end{array}\right] .
$$

We have also $\varnothing \stackrel{\text { a }}{\leq} \varnothing$, and indeed the notion of a map $\beta: \varnothing \rightarrow \varnothing$ apportioning $\varnothing$ into $\varnothing$ is trivial. For $\pi \in$ $\Pi^{*}(E) \backslash\{\varnothing\}$, we cannot have $\pi \stackrel{\text { a }}{<} \varnothing$ or $\varnothing \stackrel{\text { a }}{<} \pi: \varnothing$ is isolated.

Given $\pi_{1}, \pi_{2} \in \Pi^{*}(E) \backslash\{\emptyset\}$ such that $\pi_{1} \stackrel{\text { a }}{\leq} \pi_{2}$, we call the apportioning index of $\pi_{1}$ into $\pi_{2}$, and write $I\left(\pi_{1}, \pi_{2}\right)$, the cardinal $\left|\pi_{1} \backslash \beta\left(\pi_{2}\right)\right|$ for any $\beta: \pi_{2} \rightarrow \pi_{1}$ that apportions $\pi_{1}$ into $\pi_{2}$; indeed that cardinal, giving the number of blocks that are apportioned to other blocks, will remain constant. We also trivially define $I(\varnothing, \varnothing)=0$. We have $I\left(\pi_{1}, \pi_{2}\right)=0 \Leftrightarrow \pi_{1}=\pi_{2}$.

Let $\pi_{0}, \pi_{1}, \pi_{2} \in \Pi^{*}(E) \backslash\{\varnothing\}, \beta_{1}: \pi_{1} \rightarrow \pi_{0}$ that apportions $\pi_{0}$ into $\pi_{1}$, and $\beta_{2}: \pi_{2} \rightarrow \pi_{1}$ that apportions $\pi_{1}$ into $\pi_{2}$. Then $\beta_{1} \beta_{2}$ apportions $\pi_{0}$ into $\pi_{2}$, and $I\left(\pi_{0}, \pi_{2}\right)=I\left(\pi_{0}, \pi_{1}\right)+I\left(\pi_{1}, \pi_{2}\right)$.

The following is a more explicit version of Lemma 8 of [16], we will use it to analyse both partial apportioning and partial merging:

Lemma 10 Let $\pi_{0}, \pi_{2} \in \Pi^{*}(E) \backslash\{\emptyset\}$ with $\pi_{0} \stackrel{\mathrm{a}}{<} \pi_{2}$, with $I\left(\pi_{0}, \pi_{2}\right)=c+d$, where $c, d>0$, and let $\beta$ apportion $\pi_{0}$ into $\pi_{2}$. Let us decompose $\pi_{0} \backslash \beta\left(\pi_{2}\right)$ into the disjoint union of two partial partitions $\pi_{c}$ and $\pi_{d}$ with $\left|\pi_{c}\right|=c$ and $\left|\pi_{d}\right|=d$; set $V=\operatorname{supp}\left(\beta\left(\pi_{2}\right)\right)$ and $W=$ $\operatorname{supp}\left(\beta\left(\pi_{2}\right) \cup \pi_{c}\right)=V \cup \operatorname{supp}\left(\pi_{c}\right)$; then $\beta(C)=C \cap V$ for any $C \in \pi_{2}$. Let $\pi_{1}=\left\{C \cap W \mid C \in \pi_{2}\right\} \cup \pi_{d}$; then $\pi_{1} \in \Pi^{*}(E)$ and $\operatorname{supp}\left(\pi_{1}\right)=\operatorname{supp}\left(\pi_{0}\right)$. Define $\beta_{d}: \pi_{2} \rightarrow$ $\pi_{1}: C \mapsto C \cap W$ and $\beta_{c}: \pi_{1} \rightarrow \pi_{0}$ by $\beta_{c}(B)=B$ for $B \in \pi_{d}$, and $\beta_{c}(C \cap W)=C \cap V$ for $C \in \pi_{2}$. Then $\beta_{d}$ apportions $\pi_{1}$ into $\pi_{2}, \pi_{1} \backslash \beta_{d}\left(\pi_{2}\right)=\pi_{d}, I\left(\pi_{1}, \pi_{2}\right)=d$, $\beta_{c}$ apportions $\pi_{0}$ into $\pi_{1}, \pi_{0} \backslash \beta_{c}\left(\pi_{1}\right)=\pi_{c}, I\left(\pi_{0}, \pi_{1}\right)=c$ and $\beta_{c} \beta_{d}=\beta$.

The covering relation corresponding to the apportioning order $\stackrel{\text { a }}{\leq}$ is $\stackrel{a}{\prec}$ : for $\pi_{1}, \pi_{2} \in \Pi^{*}(E) \backslash\{\varnothing\}$, we 
write $\pi_{1} \stackrel{a}{\prec} \pi_{2}$ and say that $\pi_{2}$ a-covers $\pi_{1}$, if $\pi_{1} \stackrel{\mathrm{a}}{<} \pi_{2}$ and $I\left(\pi_{1}, \pi_{2}\right)=1$, in other words, $\pi_{2}$ is obtained by apportioning a single block of $\pi_{1}$ among remaining blocks. An equivalent formulation is:

$\pi_{1} \stackrel{\mathrm{a}}{\prec} \pi_{2} \Longleftrightarrow\left[\begin{array}{l}\operatorname{supp}\left(\pi_{1}\right)=\operatorname{supp}\left(\pi_{2}\right) \&\left|\pi_{1}\right| \geq 2 \\ \& \exists A \in \pi_{1}, \pi_{1} \backslash\{A\} \stackrel{\mathrm{i}}{<} \pi_{2}\end{array}\right]$.

When $E$ is finite, the order $\stackrel{\text { a }}{\leq}$ is graded by $h_{m}$, and for $\pi_{1} \stackrel{\text { a }}{\leq} \pi_{2}$, we have $I\left(\pi_{1}, \pi_{2}\right)=h_{m}\left(\pi_{2}\right)-h_{m}\left(\pi_{1}\right)$.

\subsection{Partial apportioning}

In order to apportion blocks between other blocks and the background, we will generalize the definition (11). Now using the isomorphism $\Pi^{*}(E) \rightarrow \Pi(\widehat{E})$ introduced in Subsection 3.1, we will also describe partial apportioning in $\Pi^{*}(E)$ as a particular case of apportioning in $\Pi(\widehat{E})$. We will then obtain maps $\beta, \alpha$ as above.

We define the partial apportioning order $\stackrel{\text { pa }}{\leq}$ as the intersection of the building order and the support containment relation: $\forall \pi_{1}, \pi_{2} \in \Pi^{*}(E)$,

$\pi_{1} \stackrel{\text { pa }}{\leq} \pi_{2} \Longleftrightarrow\left[\pi_{1} \Subset \pi_{2} \& \operatorname{supp}\left(\pi_{1}\right) \supseteq \operatorname{supp}\left(\pi_{2}\right)\right]$.

Compared to (11), we have support containment instead of support equality. We can better understand it through the isomorphism $\Pi^{*}(E) \rightarrow \Pi(\widehat{E})$ :

Lemma 11 For any $\pi_{1}, \pi_{2} \in \Pi^{*}(E), \pi_{1} \stackrel{\text { pa }}{\leq} \pi_{2}$ if and only if $\widehat{\pi_{1}} \stackrel{\mathrm{a}}{\leq} \widehat{\pi}_{2}$ and $\mathrm{Cl}_{\widehat{\pi_{1}}}(\wp) \subseteq \mathrm{Cl}_{\widehat{\pi_{2}}}(\wp)$.

Proof We have $\mathrm{Cl}_{\widehat{\pi}_{i}}(\wp)=\widehat{\operatorname{back}}\left(\pi_{i}\right)=\operatorname{back}\left(\pi_{i}\right) \cup\{\wp\}$ $(i=1,2)$. Thus $\mathrm{Cl}_{\widehat{\pi_{1}}}(\wp) \subseteq \mathrm{Cl}_{\widehat{\pi_{2}}}(\wp) \Leftrightarrow \operatorname{back}\left(\pi_{1}\right) \subseteq$ $\operatorname{back}\left(\pi_{2}\right) \Leftrightarrow \operatorname{supp}\left(\pi_{1}\right) \supseteq \operatorname{supp}\left(\pi_{2}\right)$.

Assume that this inclusion holds. If $\pi_{1} \Subset \pi_{2}$, then every block of $\pi_{2}$ contains a block of $\pi_{1}$, and as $\mathrm{Cl}_{\widehat{\pi_{2}}}(\wp)$ contains $\mathrm{Cl}_{\widehat{\pi_{1}}}(\wp)$, every block of $\widehat{\pi_{2}}$ contains a block of $\widehat{\pi_{1}}$, thus $\widehat{\pi_{1}} \Subset \widehat{\pi_{2}}$; as $\operatorname{supp}\left(\widehat{\pi_{1}}\right)=\operatorname{supp}\left(\widehat{\pi_{2}}\right)=\widehat{E}$, we deduce that $\widehat{\pi_{1}} \leq \widehat{\pi}_{2}$. Conversely, if $\widehat{\pi_{1}} \leq \widehat{\pi}_{2}$, then $\widehat{\pi_{1}} \Subset$ $\widehat{\pi_{2}}=\pi_{2} \cup\left\{\widehat{\operatorname{back}}\left(\pi_{2}\right)\right\}$, hence every block $B$ of $\pi_{2}$ contains a block of $\widehat{\pi_{1}}=\pi_{1} \cup\left\{\mathrm{Cl}_{\widehat{\pi_{1}}}(\wp)\right\}$; as $\wp \in \mathrm{Cl}_{\widehat{\pi_{1}}}(\wp)$ but $\wp \notin B, B$ does not contain $\mathrm{Cl}_{\widehat{\pi_{1}}}(\wp)$, thus it contains a block of $\pi_{1}$, that is, $\pi_{1} \Subset \pi_{2}$.

We have shown that $\operatorname{supp}\left(\pi_{1}\right) \supseteq \operatorname{supp}\left(\pi_{2}\right)$ and $\pi_{1} \Subset$ $\pi_{2}$ iff $\mathrm{Cl}_{\widehat{\pi_{1}}}(\wp) \subseteq \mathrm{Cl}_{\widehat{\pi_{2}}}(\wp)$ and $\widehat{\pi_{1}} \stackrel{\mathrm{a}}{\leq} \widehat{\pi}_{2}$.

We have then a map $\widehat{\beta}: \widehat{\pi_{2}} \rightarrow \widehat{\pi_{1}}$ apportioning $\widehat{\pi_{1}}$ into $\widehat{\pi_{2}}$; thus for every $C \in \widehat{\pi_{2}}, \widehat{\beta}(C) \in \widehat{\pi_{1}}$ and $\widehat{\beta}(C) \subseteq$ $C$. Note that even if $\mathrm{Cl}_{\widehat{\pi_{2}}}(\wp)$ contains, in addition to

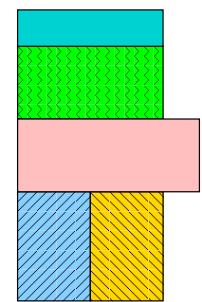

$\pi_{1}$

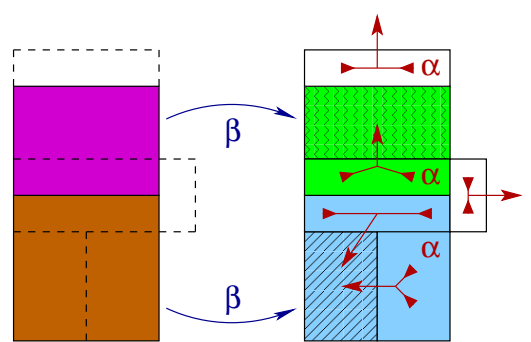

$\pi_{2}$
Fig. 14 Left: $\pi_{1}$; middle: $\pi_{2}$; here $\pi_{1} \stackrel{\text { pa }}{\leq} \pi_{2}$, the blocks of $\pi_{1}$ included in a block of $\pi_{2}$ are hatched. Right: one possible choice for $\beta$, and the resulting $\alpha$, in order to partially apportion $\pi_{1}$ into $\pi_{2}$.

$\mathrm{Cl}_{\widehat{\pi_{1}}}(\wp)$, a block of $\pi_{1}$, we do not allow a choice for $\widehat{\beta}\left(\mathrm{Cl}_{\widehat{\pi_{2}}}(\wp)\right)$, we always set $\widehat{\beta}\left(\mathrm{Cl}_{\widehat{\pi_{2}}}(\wp)\right)=\mathrm{Cl}_{\widehat{\pi_{1}}}(\wp)$. Thus the restriction $\beta$ of $\widehat{\beta}$ to $\pi_{2}$ is a map $\pi_{2} \rightarrow \pi_{1}$ satisfying $\beta(C) \subseteq C$ for all $C \in \pi_{2} ;$ conversely, if $\operatorname{supp}\left(\pi_{1}\right) \supseteq$ $\operatorname{supp}\left(\pi_{2}\right)$, any map $\beta: \pi_{2} \rightarrow \pi_{1}$ satisfying $\beta(C) \subseteq C$ for all $C \in \pi_{2}$, is the restriction of a map $\widehat{\beta}$ apportioning $\widehat{\pi_{1}}$ into $\widehat{\pi_{2}}$ : set $\widehat{\beta}(C)=\beta(C)$ for $C \in \pi_{2}$, and $\widehat{\beta}\left(\widehat{\operatorname{back}}\left(\pi_{2}\right)\right)=$ $\widehat{\operatorname{back}}\left(\pi_{1}\right)$; we say that $\beta$ partially apportions $\pi_{1}$ into $\pi_{2}$. Then we have a map $\alpha$ defined on

$$
\operatorname{supp}\left(\widehat{\pi_{1}} \backslash \widehat{\beta}\left(\widehat{\pi_{2}}\right)\right)=\operatorname{supp}\left(\pi_{1} \backslash \beta\left(\pi_{2}\right)\right)
$$

with values in

$$
\widehat{\beta}\left(\widehat{\pi_{2}}\right)=\beta\left(\pi_{2}\right) \cup\left\{\widehat{\operatorname{back}}\left(\pi_{1}\right)\right\},
$$

given by $\alpha(p)=\widehat{\beta}\left(\mathrm{Cl}_{\widehat{\pi_{2}}}(p)\right)$, cf. (10); it describes how each block of $\pi_{1} \backslash \beta\left(\pi_{2}\right)$ is apportioned between the blocks of $\beta\left(\pi_{2}\right)$ and the background $\mathrm{Cl}_{\widehat{\pi_{1}}}(\wp)=\widehat{\operatorname{back}}\left(\pi_{1}\right)$ : for $B \in \pi_{1} \backslash \beta\left(\pi_{2}\right)$, every $p \in B$ is added to a block of $\pi_{1}$ or its background, namely to $\alpha(p) \in \widehat{\beta}\left(\widehat{\pi_{2}}\right)$. See Figure 14. In particular, a whole block of $\pi_{1}$ can be merged with another block, or merged with the background, that is, removed.

We now define the partial apportioning index of $\pi_{1}$ into $\pi_{2}$, namely

$$
I\left(\pi_{1}, \pi_{2}\right)=\left|\pi_{1} \backslash \beta\left(\pi_{2}\right)\right|=\left|\widehat{\pi_{1}} \backslash \widehat{\beta}\left(\widehat{\pi_{2}}\right)\right|=I\left(\widehat{\pi_{1}}, \widehat{\pi_{2}}\right),
$$

in other words, the partial apportioning index of $\pi_{1}$ into $\pi_{2}$ equals the apportioning index of $\widehat{\pi_{1}}$ into $\widehat{\pi_{2}}$, it does not depend on the choice of $\widehat{\beta}$. It gives the number of blocks that are partially apportioned to other blocks.

Given $\pi_{0}, \pi_{1}, \pi_{2} \in \Pi^{*}(E) \backslash\{\varnothing\}, \beta_{1}: \pi_{1} \rightarrow \pi_{0}$ that partially apportions $\pi_{0}$ into $\pi_{1}$, and $\beta_{2}: \pi_{2} \rightarrow \pi_{1}$ that partially apportions $\pi_{1}$ into $\pi_{2}$, then $\beta_{1} \beta_{2}$ partially apportions $\pi_{0}$ into $\pi_{2}$, and $I\left(\pi_{0}, \pi_{2}\right)=I\left(\pi_{0}, \pi_{1}\right)+$ $I\left(\pi_{1}, \pi_{2}\right)$. This follows from the similar statement about apportioning by applying Lemma 11. 
Now we can define the corresponding covering relation: given $\pi_{1}, \pi_{2} \in \Pi^{*}(E) \backslash\{\varnothing\}$, we write $\pi_{1} \stackrel{\text { pa }}{\prec} \pi_{2}$ and say that $\pi_{2}$ pa-covers $\pi_{1}$, if $\pi_{1} \stackrel{\text { pa }}{<} \pi_{2}$ and $I\left(\pi_{1}, \pi_{2}\right)=1$. Following (12), it means that

$$
\begin{gathered}
\operatorname{supp}\left(\pi_{1}\right) \supseteq \operatorname{supp}\left(\pi_{2}\right) \&\left|\widehat{\pi_{1}}\right| \geq 2 \\
\& \exists A \in \pi_{1}, \widehat{\pi_{1}} \backslash\{A\} \stackrel{\mathrm{i}}{<} \widehat{\pi_{2}} .
\end{gathered}
$$

We get thus

$\pi_{1} \stackrel{\text { pa }}{\prec} \pi_{2} \Longleftrightarrow\left[\begin{array}{l}\operatorname{supp}\left(\pi_{1}\right) \supseteq \operatorname{supp}\left(\pi_{2}\right) \& \pi_{1} \neq \varnothing \\ \& \exists A \in \pi_{1}, \pi_{1} \backslash\{A\} \stackrel{\mathrm{i}}{\leq} \pi_{2}\end{array}\right]$.

Here the block $A$ is apportioned between the remaining blocks of $\pi_{1}$ and the background. Note that we had $\pi_{1} \backslash\{A\} \stackrel{\mathrm{i}}{<} \pi_{2}$ in (12), while we have here $\pi_{1} \backslash\{A\} \stackrel{\mathrm{i}}{\leq} \pi_{2}$; indeed the case $\pi_{1} \backslash\{A\}=\pi_{2}$, i.e., $\pi_{2}$ is obtained by removing a block of $\pi_{1}$, gives $\operatorname{supp}\left(\pi_{1}\right) \supset \operatorname{supp}\left(\pi_{2}\right)$, that is, $\mathrm{Cl}_{\widehat{\pi_{1}}}(\wp) \subset \mathrm{Cl}_{\widehat{\pi_{2}}}(\wp)$, so $\widehat{\pi_{1}} \backslash\{A\} \stackrel{\mathrm{i}}{<} \widehat{\pi_{2}}$. Moreover, when $\left|\pi_{1}\right|=1$, we get $\pi_{1}=\{A\}$ and $\pi_{2}=\pi_{1} \backslash\{A\}=\varnothing$, so the unique block $A$ is removed from $\pi_{1}$.

The following analogue of Lemma 10 will be used for both the partial apportioning and partial merging orders:

Lemma 12 Let $\pi_{0}, \pi_{2} \in \Pi^{*}(E)$ such that $\widehat{\pi_{0}} \stackrel{\text { a }}{<} \widehat{\pi_{2}}$, with $I\left(\widehat{\pi_{0}}, \widehat{\pi_{2}}\right)=c+d$, where $c, d>0$, and let $\widehat{\beta}$ apportion $\widehat{\pi_{0}}$ into $\widehat{\pi_{2}}$. Let $\widehat{\pi_{1}} \in \Pi(\widehat{E}), \widehat{\beta_{d}}: \widehat{\pi_{2}} \rightarrow \widehat{\pi_{1}}$ and $\widehat{\beta_{c}}: \widehat{\pi_{1}} \rightarrow \widehat{\pi_{0}}$ be constructed as in Lemma 10, and let $\pi_{1} \in \Pi^{*}(E)$ correspond to $\widehat{\pi_{1}}$. Then:

1. If $\widehat{\beta}\left(\mathrm{Cl}_{\widehat{\pi_{2}}}(\wp)\right)=\mathrm{Cl}_{\widehat{\pi_{0}}}(\wp)$, then $\widehat{\beta_{d}}\left(\mathrm{Cl}_{\widehat{\pi_{2}}}(\wp)\right)=\mathrm{Cl}_{\widehat{\pi_{1}}}(\wp)$ and $\widehat{\beta}_{c}\left(\mathrm{Cl}_{\widehat{\pi_{1}}}(\wp)\right)=\mathrm{Cl}_{\widehat{\pi_{0}}}(\wp)$, in particular $\mathrm{Cl}_{\widehat{\pi_{0}}}(\wp) \subseteq$ $\mathrm{Cl}_{\widehat{\pi_{1}}}(\wp) \subseteq \mathrm{Cl}_{\widehat{\pi_{2}}}(\wp)$.

2. If $\pi_{0} \vdash \pi_{2}$, then $\pi_{1} \vdash \pi_{2}$.

3. If $\pi_{0} \vdash \pi_{2}$ and $\widehat{\beta}\left(\mathrm{Cl}_{\widehat{\pi_{2}}}(\wp)\right)=\mathrm{Cl}_{\widehat{\pi_{0}}}(\wp)$, then $\pi_{0} \vdash \pi_{1}$.

Proof 1. As $\widehat{\beta_{d}}$ apportions $\widehat{\pi_{1}}$ into $\widehat{\pi_{2}}, \widehat{\beta_{c}}$ apportions $\widehat{\pi_{0}}$ into $\widehat{\pi_{1}}$ and $\widehat{\beta}=\widehat{\beta}_{c} \widehat{\beta_{d}}$, we get

$$
\begin{aligned}
\wp \in \mathrm{Cl}_{\widehat{\pi_{0}}}(\wp) & =\widehat{\beta}\left(\mathrm{Cl}_{\widehat{\pi_{2}}}(\wp)\right)=\widehat{\beta_{c}} \widehat{\beta_{d}}\left(\mathrm{Cl}_{\widehat{\pi_{2}}}(\wp)\right) \\
\subseteq \widehat{\beta_{d}} & \left(\mathrm{Cl}_{\widehat{\pi_{2}}}(\wp)\right) \in \widehat{\pi_{1}} ;
\end{aligned}
$$

hence $\mathrm{Cl}_{\widehat{\pi_{1}}}(\wp)=\widehat{\beta_{d}}\left(\mathrm{Cl}_{\widehat{\pi_{2}}}(\wp)\right) \subseteq \mathrm{Cl}_{\widehat{\pi_{2}}}(\wp)$ and $\mathrm{Cl}_{\widehat{\pi_{0}}}(\wp)=$ $\widehat{\beta_{c}} \widehat{\beta_{d}}\left(\mathrm{Cl}_{\widehat{\pi_{2}}}(\wp)\right)=\widehat{\beta}_{c}\left(\mathrm{Cl}_{\widehat{\pi_{1}}}(\wp)\right) \subseteq \mathrm{Cl}_{\widehat{\pi_{1}}}(\wp)$.

2. From the construction in Lemma 10, we have $\widehat{\pi_{1}}=\left\{C \cap W \mid C \in \widehat{\pi_{2}}\right\} \cup \widehat{\pi_{d}}$, where $\widehat{\pi_{d}} \subseteq \widehat{\pi_{0}}$. Let $B \in \pi_{1}$. If $B=C \cap W$ for some $C \in \widehat{\pi_{2}}$, as $C \cap W \subseteq C$, $B=C \cap W$ does not overlap any other block of $\widehat{\pi_{2}}$, so it overlaps at most one block of $\pi_{2}$. If $B \in \widehat{\pi_{d}}$, then $B \in \widehat{\pi_{0}}$, and as $\wp \notin B, B \in \pi_{0}$, and since $\pi_{0} \vdash \pi_{2}, B$ overlaps at most one block of $\pi_{2}$. Therefore $\pi_{1} \vdash \pi_{2}$.
3. From the construction in Lemma 10, we have both $\widehat{\pi_{d}} \subseteq \widehat{\pi_{0}}$ and $\widehat{\pi_{d}} \subseteq \widehat{\pi_{1}}$. Let $A \in \pi_{0}$. If $A \in \widehat{\pi_{d}}$, then $A \in \widehat{\pi_{1}}$, so $A$ is disjoint from any other block of $\widehat{\pi_{1}}$, thus it overlaps no other block of $\pi_{1}$ than itself. If $A \notin \widehat{\pi_{d}}$, then $A$ overlaps no block of $\widehat{\pi_{d}}$, so the only blocks of $\widehat{\pi_{1}}$ that it can overlap are the $C \cap W=\widehat{\beta_{d}}(C)$ for $C \in \widehat{\pi_{2}}$; if $A$ overlaps two of them, $C_{1} \cap W$ and $C_{2} \cap W$, then it overlaps both $C_{1}, C_{2} \in \widehat{\pi_{2}}$, and as $\pi_{0} \vdash \pi_{2}$, we cannot have both $C_{1}$ or $C_{2}$ in $\pi_{2}$, so for $i=1$ or 2 we have $C_{i}=\mathrm{Cl}_{\widehat{\pi_{2}}}(\wp) ;$ as $\widehat{\beta}\left(\mathrm{Cl}_{\widehat{\pi_{2}}}(\wp)\right)=\mathrm{Cl}_{\widehat{\pi_{0}}}(\wp)$, item 1 gives

$$
C_{i} \cap W=\widehat{\beta_{d}}\left(C_{i}\right)=\widehat{\beta_{d}}\left(\mathrm{Cl}_{\widehat{\pi_{2}}}(\wp)\right)=\mathrm{Cl}_{\widehat{\pi_{1}}}(\wp) \text {, }
$$

so we cannot have both $C_{1} \cap W$ and $C_{2} \cap W$ in $\pi_{1}$, therefore $\pi_{0} \vdash \pi_{1}$.

Theorem 13 Partial apportioning $\stackrel{\text { pa }}{\leq}$ is a partial order relation on $\Pi^{*}(E)$; it is included in the building order $\Subset$ and it contains the apportioning $\stackrel{\text { a }}{\leq}$ and regional $\stackrel{\mathrm{rg}}{\leq}$ orders; in other words, for any $\pi_{1}, \pi_{2} \in \Pi^{*}(E), \pi_{1} \underset{\mathrm{rg}}{\mathrm{pa}} \pi_{2}$ implies $\pi_{1} \Subset \pi_{2}$ and each of $\pi_{1} \stackrel{\text { a }}{\leq} \pi_{2}$ and $\pi_{1} \stackrel{\text { rg }}{\leq} \pi_{2}$ implies $\pi_{1} \stackrel{\text { pa }}{\leq} \pi_{2}$. The greatest and least elements are $\emptyset$ and $\mathbf{0}_{E}$. The covering relation is $\stackrel{\text { pa }}{\prec}$.

Let $E$ be finite. Then $\left(\Pi^{*}(E), \stackrel{\text { pa }}{\leq}\right)$ is graded by $h_{r}$, or equivalently by $-h_{c}$, that is, for any $\pi_{1}, \pi_{2} \in \Pi^{*}(E)$ we have

$$
\begin{aligned}
& \pi_{1} \stackrel{\text { pa }}{<} \pi_{2} \Longrightarrow h_{c}\left(\pi_{1}\right)>h_{c}\left(\pi_{2}\right), \\
& \pi_{1} \stackrel{\text { pa }}{\prec} \pi_{2} \Longrightarrow h_{c}\left(\pi_{2}\right)=h_{c}\left(\pi_{1}\right)-1 .
\end{aligned}
$$

For $\pi \in \Pi^{*}(E)$, the height of $\pi$ is $h_{r}(\pi)$. For $\pi_{1}, \pi_{2} \in$ $\Pi^{*}(E)$ such that $\pi_{1} \leq \pi_{2}$, we have $I\left(\pi_{1}, \pi_{2}\right)=h_{r}\left(\pi_{2}\right)-$ $h_{r}\left(\pi_{1}\right)=h_{c}\left(\pi_{1}\right)-h_{c}\left(\pi_{2}\right)$.

Proof Since the building order $\Subset$ is a partial order and the support containment relation is a quasi-order, their intersection $\stackrel{\text { pa }}{\leq}$ is an order. By definition (13), $\pi_{1} \stackrel{\text { pa }}{\leq} \pi_{2}$ implies $\pi_{1} \Subset \pi_{2}$. Comparing (11) with (13), clearly $\pi_{1} \stackrel{\text { a }}{\leq}$ $\pi_{2}$ is a particular case of $\pi_{1} \stackrel{\text { pa }}{\leq} \pi_{2}$. If $\pi_{1} \stackrel{\text { rg }}{\leq} \pi_{2}$, then by Proposition $4, \operatorname{supp}\left(\pi_{1}\right) \supseteq \operatorname{supp}\left(\pi_{2}\right)$ and every block of $\pi_{2}$ is the union of some blocks of $\pi_{1}$, so it contains at least one such block, that is, $\pi_{1} \Subset \pi_{2}$; thus $\pi_{1} \stackrel{\text { pa }}{\leq} \pi_{2}$.

By Theorem 5, for any $\pi \in \Pi^{*}(E)$, we have $\mathbf{0}_{E} \stackrel{\text { rg }}{\leq}$ $\pi \stackrel{\text { rg }}{\leq} \emptyset$, and as $\stackrel{\text { pa }}{\leq}$ contains $\stackrel{\text { rg }}{\leq}$, we derive that $\mathbf{0}_{E} \stackrel{\text { pa }}{\leq} \pi \stackrel{\text { pa }}{\leq}$ $\varnothing$, so $\varnothing$ and $\mathbf{0}_{E}$ are the greatest and least elements.

Let $\pi_{0}, \pi_{2} \in \Pi^{*}(E)$ such that $\pi_{0} \stackrel{\text { pa }}{<} \pi_{2}$; then $\pi_{0} \neq \varnothing$, $\widehat{\pi_{0}} \stackrel{\mathrm{a}}{<} \widehat{\pi_{2}}$ and $I\left(\pi_{0}, \pi_{2}\right)=I\left(\widehat{\pi_{0}}, \widehat{\pi_{2}}\right)>0$. If $\pi_{2}$ does not cover $\pi_{0}$, then for some $\pi_{1} \in \Pi^{*}(E)$ we have $\pi_{0} \stackrel{\text { pa }}{<} \pi_{1} \stackrel{\text { pa }}{<}$ $\pi_{2}$, hence $\widehat{\pi_{0}} \stackrel{\mathrm{a}}{<} \widehat{\pi_{1}} \stackrel{\mathrm{a}}{<} \widehat{\pi_{2}}$, so $I\left(\widehat{\pi_{0}}, \widehat{\pi_{1}}\right)>0, I\left(\widehat{\pi_{1}}, \widehat{\pi_{2}}\right)>0$ and $I\left(\widehat{\pi_{0}}, \widehat{\pi_{2}}\right)=I\left(\widehat{\pi_{0}}, \widehat{\pi_{1}}\right)+\left(\widehat{\pi_{1}}, \widehat{\pi_{2}}\right)>1$. Conversely, 
if $I\left(\widehat{\pi_{0}}, \widehat{\pi_{2}}\right)>1$, let $\widehat{\beta}: \widehat{\pi_{2}} \rightarrow \widehat{\pi_{0}}$ that apportions $\widehat{\pi_{0}}$ into $\widehat{\pi_{2}}$, with $\widehat{\beta}\left(\mathrm{Cl}_{\widehat{\pi_{2}}}(\wp)\right)=\mathrm{Cl}_{\widehat{\pi_{0}}}(\wp)$. Set $c=1$ and $d=$ $I\left(\widehat{\pi_{0}}, \widehat{\pi_{2}}\right)-1$, so $c, d>0$, and we apply Lemma 10: there is some $\widehat{\pi_{1}} \in \Pi(\widehat{E})$ (corresponding to $\pi_{1} \in \Pi^{*}(E)$ ), two maps $\widehat{\beta_{d}}: \widehat{\pi_{2}} \rightarrow \widehat{\pi_{1}}$ and $\widehat{\beta_{c}}: \widehat{\pi_{1}} \rightarrow \widehat{\pi_{0}}$ such that $\widehat{\beta_{c}}$ apportions $\widehat{\pi_{0}}$ into $\widehat{\pi_{1}}, I\left(\widehat{\pi_{0}}, \widehat{\pi_{1}}\right)=c, \widehat{\beta_{d}}$ apportions $\widehat{\pi_{1}}$ into $\widehat{\pi_{2}}, I\left(\widehat{\pi_{1}}, \widehat{\pi_{2}}\right)=d$ and $\widehat{\beta}=\widehat{\beta}_{c} \widehat{\beta_{d}}$. We have thus $\widehat{\pi_{0}} \stackrel{\mathrm{a}}{<} \widehat{\pi_{1}} \stackrel{\mathrm{a}}{<} \widehat{\pi_{2}}$ and item 1 of Lemma 12 gives $\mathrm{Cl}_{\widehat{\pi_{0}}}(\wp) \subseteq \mathrm{Cl}_{\widehat{\pi_{1}}}(\wp) \subseteq \mathrm{Cl}_{\widehat{\pi_{2}}}(\wp)$. Therefore $\pi_{0} \stackrel{\text { pa }}{<} \pi \stackrel{\text { pa }}{<} \pi_{2}$. We have thus shown that for $\pi_{0} \stackrel{\text { pa }}{<} \pi_{2}, I\left(\widehat{\pi_{0}}, \widehat{\pi_{2}}\right)>0$, where $I\left(\widehat{\pi_{0}}, \widehat{\pi_{2}}\right)>1$ if and only if $\pi_{2}$ does not cover $\pi_{0}$, in other words, $\pi_{2}$ covers $\pi_{0}$ if and only if $I\left(\pi_{0}, \pi_{2}\right)=$ $I\left(\widehat{\pi_{0}}, \widehat{\pi_{2}}\right)=1$, that is, $\pi_{0} \stackrel{\text { pa }}{\prec} \pi_{2}$, so $\stackrel{\text { pa }}{\prec}$ is the covering relation.

Let $E$ be finite. By [16], $\Pi(\widehat{E})$, ordered by $\stackrel{\text { a }}{\leq}$ is graded by $h_{m}$, and (6) gives $h_{m}(\widehat{\pi})=|E|-h_{c}(\pi)=$ $h_{r}(\pi)$ for all $\pi \in \Pi^{*}(E)$. Hence for any $\pi_{1}, \pi_{2} \in \Pi^{*}(E)$ we have

$$
\begin{gathered}
\pi_{1} \stackrel{\text { pa }}{<} \pi_{2} \Rightarrow \widehat{\pi_{1}} \stackrel{\mathrm{a}}{<} \widehat{\pi_{2}} \Rightarrow h_{m}\left(\widehat{\pi_{1}}\right)<h_{m}\left(\widehat{\pi_{2}}\right) \\
\Rightarrow h_{r}\left(\pi_{1}\right)<h_{r}\left(\pi_{2}\right) \Rightarrow h_{c}\left(\pi_{1}\right)>h_{c}\left(\pi_{2}\right)
\end{gathered}
$$

and

$$
\begin{gathered}
\pi_{1} \stackrel{\text { pa }}{\prec} \pi_{2} \Rightarrow \widehat{\pi_{1}} \stackrel{\mathrm{a}}{\prec} \widehat{\pi_{2}} \Rightarrow h_{m}\left(\widehat{\pi_{2}}\right)=h_{m}\left(\widehat{\pi_{1}}\right)+1 \\
\Rightarrow h_{r}\left(\pi_{2}\right)=h_{r}\left(\pi_{1}\right)+1 \Rightarrow h_{c}\left(\pi_{2}\right)=h_{c}\left(\pi_{1}\right)-1,
\end{gathered}
$$

so $\left(\Pi^{*}(E), \stackrel{\text { pa }}{\leq}\right)$ is graded by $h_{r}$, equivalently by $-h_{c}$. For $\pi_{1} \stackrel{\text { pa }}{\leq} \pi_{2}, \widehat{\pi_{1}} \stackrel{\text { a }}{\leq} \widehat{\pi_{2}}$, and [16] again gives $I\left(\widehat{\pi_{1}}, \widehat{\pi_{2}}\right)=$ $h_{m}\left(\widehat{\pi_{2}}\right)-h_{m}\left(\widehat{\pi_{1}}\right)$, hence

$$
I\left(\pi_{1}, \pi_{2}\right)=h_{r}\left(\pi_{2}\right)-h_{r}\left(\pi_{1}\right)=h_{c}\left(\pi_{1}\right)-h_{c}\left(\pi_{2}\right) .
$$

As $\mathbf{0}_{E}$ is the least element, the height of $\pi \in \Pi^{*}(E)$ is $h_{r}(\pi)-h_{r}\left(\mathbf{0}_{E}\right)=h_{r}(\pi)-0=h_{r}(\pi)$.

We know from [16] that the apportioning order $\stackrel{a}{\leq}$ contains the merging order $\stackrel{\mathrm{m}}{\leq}$, and from Theorem 5 that the regional order $\stackrel{\text { rg }}{\leq}$ contains the merging ${ }^{m} \leq$ and inverse inclusion $\supseteq$ orders; thus the partial apportioning order $\stackrel{\text { pa }}{\leq}$ contains also the merging $\stackrel{m}{\leq}$ and inverse inclusion $\supseteq$ orders.

\subsection{Partial merging}

We will consider the intersection of the partial apportioning $\stackrel{\text { pa }}{\leq}$ and linking $\stackrel{\ln }{\leq}$ orders, we call it the partial merging order, and write it $\stackrel{\mathrm{pm}}{\leq}$. Thus by $(8,13)$ we have for any $\pi_{1}, \pi_{2} \in \Pi^{*}(E)$ :

$$
\pi_{1} \stackrel{\mathrm{pm}}{\leq} \pi_{2} \Longleftrightarrow\left[\begin{array}{l}
\pi_{1} \Subset \pi_{2} \& \pi_{1} \vdash \pi_{2} \& \\
\operatorname{supp}\left(\pi_{1}\right) \supseteq \operatorname{supp}\left(\pi_{2}\right)
\end{array}\right] .
$$

We will describe it from the both points of views of a map $\beta$ that partially apportions $\pi_{1}$ into $\pi_{2}$ and of a map $\lambda$ that links $\pi_{1}$ to $\pi_{2}$.

Let $\pi_{1} \stackrel{\text { pm }}{\leq} \pi_{2} ;$ since $\pi_{1} \stackrel{\text { pa }}{\leq} \pi_{2}$, we can describe the relation between $\pi_{1}$ and $\pi_{2}$ in terms of the map $\beta$ : $\pi_{2} \rightarrow \pi_{1}$ that partially apportions $\pi_{1}$ into $\pi_{2}$, the map $\alpha: \operatorname{supp}\left(\pi_{1} \backslash \beta\left(\pi_{2}\right)\right) \rightarrow \beta\left(\pi_{2}\right) \cup\left\{\widehat{\operatorname{back}}\left(\pi_{1}\right)\right\}$ and the partial apportioning index of $\pi_{1}$ into $\pi_{2}, I\left(\pi_{1}, \pi_{2}\right)=$ $\left|\pi_{1} \backslash \beta\left(\pi_{2}\right)\right|$. For every block $A \in \pi_{1}$, as $\pi_{1} \vdash \pi_{2}, A$ overlaps at most one block of $\pi_{2}$, so $A \cap \operatorname{supp}\left(\pi_{2}\right)$ is either empty or included in a block of $\pi_{2}$. More precisely, we have one of the following three possibilities:

1. There is a block $B \in \pi_{2}$ such that $A \subseteq B$; this can be because either $A=\beta(B)$ or $A \notin \beta\left(\pi_{2}\right)$ and $A$ is merged with $\beta(B)$, that is, $\alpha(p)=\beta(B)$ for all $p \in A$.

2. $A \subseteq \operatorname{back}\left(\pi_{2}\right)$; here $A \notin \beta\left(\pi_{2}\right)$ and $A$ is merged with the background, that is, $\alpha(p)=\widehat{\operatorname{back}}\left(\pi_{1}\right)$ for all $p \in A$.

3. $\operatorname{supp}\left(\pi_{2}\right) \succ A \gamma \operatorname{back}\left(\pi_{2}\right)$ and there is a block $B \in \pi_{2}$ such that $A \cap \operatorname{supp}\left(\pi_{2}\right) \subseteq B$; here $A \notin \beta\left(\pi_{2}\right)$ and we have $\alpha(p)=\beta(B)$ for $p \in A \cap \operatorname{supp}\left(\pi_{2}\right)$ and $\alpha(p)=\widehat{\operatorname{back}}\left(\pi_{1}\right)$ for $p \in A \cap \operatorname{back}\left(\pi_{2}\right)$.

In other words, a block of $\pi_{1}$ either is included in a block of $\pi_{2}$, or is removed (goes to the background back $\left(\pi_{2}\right)$ ), or is split into two parts, one being removed (merging with the background), and the other is merged with another block of $\pi_{1}$; in the third case, we say that the block is partially merged. See Figure 5 .

Compared with the general case of $\pi_{1} \leq \pi_{2}$, we exclude the case where for some $k \geq 2$, there are $k$ blocks $B_{1}, \ldots, B_{k} \in \pi_{2}$ overlapping $A$, and $A$ is apportioned between either the blocks $\beta\left(B_{1}\right), \ldots, \beta\left(B_{k}\right) \in \pi_{1}$, or the blocks $\beta\left(B_{1}\right), \ldots, \beta\left(B_{k}\right)$ and the background $\widehat{\operatorname{back}}\left(\pi_{1}\right)$.

We can now interpret the partial merging order $\pi_{1} \stackrel{\text { pm }}{\leq}$ $\pi_{2}$ in terms of a map that links $\pi_{1}$ to $\pi_{2}$. As in Lemma 6 and in the proof of Proposition 8, we define

$$
\pi=\left\{A \in \pi_{1} \mid \exists C \in \pi_{2}, A \gamma C\right\},
$$

the set of blocks of $\pi_{1}$ that overlap some block of $\pi_{2}$, and $\lambda: \pi \rightarrow \pi_{2}$ by $\lambda(A)=C$, for the unique block $C \in \pi_{2}$ such that $A \gamma C$. Then from the linking order $\pi_{1} \stackrel{\text { In }}{\leq} \pi_{2}$ we have $C \subseteq \bigcup\{A \in \pi \mid \lambda(A)=C\}$ for every $C \in \pi_{2}$. But we have also $\pi_{1} \Subset \pi_{2}$, in other words every block of $\pi_{2}$ contains a block of $\pi_{1}$, which means that for every $C \in \pi_{2}$, there is some $A \in \pi$ such that $\lambda(A)=C$ and $A \subseteq C$.

Comparing the two descriptions in terms of the map $\lambda$ the and two maps $\beta, \alpha$, we have $\beta\left(\pi_{2}\right) \subseteq \pi$ and for any $B \in \beta\left(\pi_{2}\right), \lambda(B)=\beta^{-1}(B)$, which is the block of $\pi_{2}$ containing $B$; for $B \in \pi \backslash \beta\left(\pi_{2}\right), \lambda(B)$ is the block of $\pi_{2}$ 


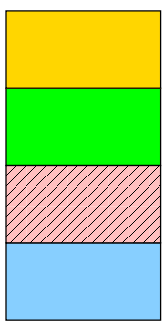

$\pi_{1}$

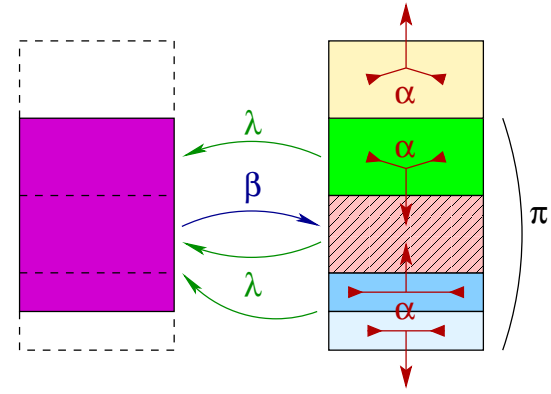

$\pi_{2}$
Fig. 15 Left: From $\pi_{1}$; middle: $\pi_{2}$; here $\pi_{1} \stackrel{\mathrm{pm}}{\leq} \pi_{2}$, and the blocks of $\pi_{1}$ to which others will be partially merged are shown hatched. Right: all portions of blocks of $\pi_{1}$ included in $\operatorname{back}\left(\pi_{2}\right)$ are shown in a lighter colour, the other portions keep their coulour; we show $\pi$, the maps $\beta, \alpha$ and $\lambda$.

containing $B \cap \operatorname{supp}\left(\pi_{2}\right)$, thus for all $p \in B \cap \operatorname{supp}\left(\pi_{2}\right)$ we have $\beta^{-1}(\alpha(p))=\lambda(B)$. For $B \in \pi_{1} \backslash \pi, B \subseteq \operatorname{back}\left(\pi_{2}\right)$. See Figure 15.

We write $\pi_{1} \stackrel{\mathrm{pm}}{\prec} \pi_{2}$ and say that $\pi_{2}$ pm-covers $\pi_{1}$, if $\pi_{1} \stackrel{\text { pm }}{<} \pi_{2}$ and $I\left(\pi_{1}, \pi_{2}\right)=1$, in other words if $\pi_{1} \stackrel{\text { pa }}{\prec} \pi_{2}$ and $\pi_{1} \vdash \pi_{2}$. In practice this means that $\pi_{1} \neq \varnothing$ and for some block $A \in \pi_{1}$, one of the following holds:

1. $\pi_{2}=\pi_{1} \backslash\{A\}: A$ is removed;

2. $\left|\pi_{1}\right| \geq 2$ and there is $B \in \pi_{1} \backslash\{A\}$ such that $\pi_{2}=$ $\left(\pi_{1} \backslash\{A, B\}\right) \cup\{A \cup B\}: A$ is merged with $B$;

3. $\left|\pi_{1}\right| \geq 2,|A| \geq 2$ and there are $C \subset A$ with $C \neq \emptyset$ and $B \in \pi_{1} \backslash\{A\}$ such that $\pi_{2}=\left(\pi_{1} \backslash\{A, B\}\right) \cup$ $\{C \cup B\}: A$ is split into two non-void parts $C$ and $A \backslash C, C$ is merged with the block $B$ and $A \backslash C$ is removed, that is, added to the background.

We will now characterize the partial merging order from the point of view of partial apportioning:

Theorem 14 Partial merging $\stackrel{\mathrm{pm}}{\leq}$ is a partial order relation on $\Pi^{*}(E)$; it contains the regional $\stackrel{\mathrm{rg}}{\leq}$ order; in other words, for any $\pi_{1}, \pi_{2} \in \Pi^{*}(E), \pi_{1} \stackrel{\mathrm{rg}}{\leq} \pi_{2}$ implies $\pi_{1} \stackrel{\mathrm{pm}}{\leq} \pi_{2}$. The greatest and least elements are $\varnothing$ and $\mathbf{0}_{E}$. The covering relation is $\stackrel{\mathrm{pm}}{\prec}$.

Let $E$ be finite. Then $\left(\Pi^{*}(E), \stackrel{\mathrm{pm}}{\leq}\right)$ is graded by $h_{r}$, or equivalently by $-h_{c}$, that is, for any $\pi_{1}, \pi_{2} \in \Pi^{*}(E)$ we have

$$
\begin{aligned}
& \pi_{1} \stackrel{\mathrm{pm}}{<} \pi_{2} \Longrightarrow h_{c}\left(\pi_{1}\right)>h_{c}\left(\pi_{2}\right), \\
& \pi_{1} \stackrel{\mathrm{pm}}{\prec} \pi_{2} \Longrightarrow h_{c}\left(\pi_{2}\right)=h_{c}\left(\pi_{1}\right)-1 .
\end{aligned}
$$

For $\pi \in \Pi^{*}(E)$, the height of $\pi$ is $h_{r}(\pi)$. For $\pi_{1}, \pi_{2} \in$ $\Pi^{*}(E)$ such that $\pi_{1} \leq \pi_{2}$, we have $I\left(\pi_{1}, \pi_{2}\right)=h_{r}\left(\pi_{2}\right)-$ $h_{r}\left(\pi_{1}\right)=h_{c}\left(\pi_{1}\right)-h_{c}\left(\pi_{2}\right)$.
Proof Being the intersection of the partial apportioning $\stackrel{\text { pa }}{\leq}$ and linking $\stackrel{\text { In }}{\leq}$ orders, both of which are partial order relation on $\Pi^{*}(E)$ containing the regional order $\stackrel{\mathrm{rg}}{\leq}$, the partial merging order $\stackrel{\mathrm{pm}}{\leq}$ will also be a partial order relation on $\Pi^{*}(E)$ containing $\stackrel{\text { rg }}{\leq}$.

For any $\pi \in \Pi^{*}(E), \mathbf{0}_{E} \stackrel{\mathrm{rg}}{\leq} \pi \stackrel{\mathrm{rg}}{\leq} \emptyset$, hence $\mathbf{0}_{E} \stackrel{\mathrm{pm}}{\leq} \pi \stackrel{\mathrm{pm}}{\leq}$ $\varnothing$, thus $\varnothing$ and $\mathbf{0}_{E}$ are the greatest and least elements.

Let $\pi_{0}, \pi_{2} \in \Pi^{*}(E)$ such that $\pi_{0} \stackrel{\text { pm }}{<} \pi_{2}$; then $\pi_{0} \stackrel{\text { pa }}{<}$ $\pi_{2}, \widehat{\pi_{0}} \stackrel{\mathrm{a}}{<} \widehat{\pi_{2}}$ and $I\left(\pi_{0}, \pi_{2}\right)=I\left(\widehat{\pi_{0}}, \widehat{\pi_{2}}\right)>0$. If $\pi_{2}$ does not cover $\pi_{0}$, then for some $\pi_{1} \in \Pi^{*}(E)$ we have $\pi_{0} \stackrel{\text { pm }}{<} \pi_{1} \stackrel{\mathrm{pm}}{<}$ $\pi_{2}$, hence $\widehat{\pi_{0}} \stackrel{\mathrm{a}}{<} \widehat{\pi_{1}} \stackrel{\mathrm{a}}{<} \widehat{\pi_{2}}$, so $I\left(\widehat{\pi_{0}}, \widehat{\pi_{1}}\right)>0, I\left(\widehat{\pi_{1}}, \widehat{\pi_{2}}\right)>0$ and $I\left(\widehat{\pi_{0}}, \widehat{\pi_{2}}\right)=I\left(\widehat{\pi_{0}}, \widehat{\pi_{1}}\right)+\left(\widehat{\pi_{1}}, \widehat{\pi_{2}}\right)>1$. Conversely, if $I\left(\widehat{\pi_{0}}, \widehat{\pi_{2}}\right)>1$, let $\widehat{\beta}: \widehat{\pi_{2}} \rightarrow \widehat{\pi_{0}}$ that apportions $\widehat{\pi_{0}}$ into $\widehat{\pi_{2}}$, with $\widehat{\beta}\left(\mathrm{Cl}_{\widehat{\pi_{2}}}(\wp)\right)=\mathrm{Cl}_{\widehat{\pi_{0}}}(\wp)$. As in the proof of Theorem 13, we obtain $\widehat{\pi_{1}} \in \Pi(\widehat{E})$ according to Lemma 10, with $\widehat{\pi_{0}} \stackrel{\text { a }}{<} \widehat{\pi_{1}} \stackrel{\text { a }}{<} \widehat{\pi_{2}}$ and $\pi_{0} \stackrel{\text { pa }}{<} \pi_{1} \stackrel{\text { pa }}{<} \pi_{2}$. As $\widehat{\beta}\left(\mathrm{Cl}_{\widehat{\pi_{2}}}(\wp)\right)=\mathrm{Cl}_{\widehat{\pi_{0}}}(\wp)$ and $\pi_{0} \vdash \pi_{2}$, items 2 and 3 of Lemma 12 give $\pi_{1} \vdash \pi_{2}$ and $\pi_{0} \vdash \pi_{1}$, hence $\pi_{0} \stackrel{\mathrm{pm}}{<} \pi \stackrel{\mathrm{pm}}{<} \pi_{2}$. We have thus shown that for $\pi_{0} \stackrel{\mathrm{pm}}{<} \pi_{2}, I\left(\widehat{\pi_{0}}, \widehat{\pi_{2}}\right)>0$, where $I\left(\widehat{\pi_{0}}, \widehat{\pi_{2}}\right)>1$ if and only if $\pi_{2}$ does not cover $\pi_{0}$, in other words, $\pi_{2}$ covers $\pi_{0}$ if and only if $I\left(\pi_{0}, \pi_{2}\right)=$ $I\left(\widehat{\pi_{0}}, \widehat{\pi_{2}}\right)=1$, that is, $\pi_{0} \stackrel{\mathrm{pm}}{\prec} \pi_{2}$, so $\stackrel{\mathrm{pm}}{\prec}$ is the covering relation.

For any $\pi_{1}, \pi_{2} \in \Pi^{*}(E)$, combining Theorem 13

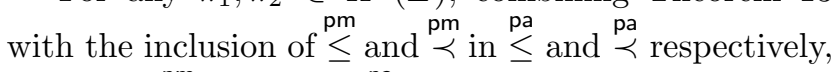
we get $\pi_{1} \stackrel{\text { pm }}{<} \pi_{2} \Rightarrow \pi_{1} \stackrel{\text { pa }}{<} \pi_{2} \Rightarrow h_{c}\left(\pi_{1}\right)>h_{c}\left(\pi_{2}\right)$ and $\pi_{1} \stackrel{\text { pm }}{\prec} \pi_{2} \Rightarrow \pi_{1} \stackrel{\text { pa }}{\prec} \pi_{2} \Rightarrow h_{c}\left(\pi_{2}\right)=h_{c}\left(\pi_{1}\right)-1$, hence $\left(\Pi^{*}(E), \stackrel{\mathrm{pm}}{\leq}\right)$ is graded by $-h_{c}$, equivalently by $h_{r}$. As in Theorem 13, we obtain the height of $\pi$ equal to $h_{r}(\pi)$ and $I\left(\pi_{1}, \pi_{2}\right)=h_{r}\left(\pi_{2}\right)-h_{r}\left(\pi_{1}\right)=h_{c}\left(\pi_{1}\right)-h_{c}\left(\pi_{2}\right)$.

We know from Theorem 5 that the regional order $\stackrel{\mathrm{rg}}{\leq}$ contains the merging $\stackrel{\mathrm{m}}{\leq}$ and inverse inclusion $\supseteq$ orders; thus the partial merging order $\stackrel{\mathrm{pm}}{\leq}$ contains also the merging $\stackrel{m}{\leq}$ and inverse inclusion $\supseteq$ orders.

5.4 Possible applications of partial apportioning and partial merging

Partial apportioning is useful in order to eliminate "parasitic" blocks in an image segmentation [20,21] in the case where this segmentation consists of a partial partition of objects against a background; in general, such "parasitic" blocks correspond to noise or lie inside transitions between homogeneous regions, themselves forming blocks (or the background). Thus "parasitic" blocks will be removed, and their contents will be apportioned 
between neighbouring blocks corresponding to significant objects, and the background, so the partial partition will grow according to the partial apportioning order.

Let us return to the example given at the end of Subsection 3.2, of a partial partition whose blocks will be rated as of high, medium or low quality. We eliminated blocks of low quality and kept those of high quality, while those of medium quality were retained only on certain conditions (for instance being adjacent to a high-quality block, as in double thresholding). Now suppose that blocks of medium quality can be subjected to a more detailed examination, and split into two parts, of medium-high and medium-low quality respectively, where the medium-low one is eliminated; now if the medium-high part is adjacent to a high quality block and their merging retains a high quality, it is merged with it, otherwise it is eliminated. We operate thus a partial merging of medium quality blocks with neighbouring high quality ones.

\section{The joining order}

We will consider the order where a partial partition is increased by any operation on blocks considered in this paper, namely deflating, removing, merging, apportioning, partial apportioning, and partial merging. In fact, all these operations can be generated by block apportioning followed by block deflating. This order will contain all other orders considered in this paper, thus any improvement of a partial partition according to one of these orders will mean a growth for this new order. Its general definition in terms of a relation between the blocks of the two partial partitions is far from intuitive, and we do not see yet possible applications for it, hence we deem this order to be less interesting than the previous ones; maybe future problems could justify it.

We define the joining order $\stackrel{\text { jn }}{\leq}$ as follows: for $\pi_{1}, \pi_{2} \in$ $\Pi^{*}(E), \pi_{1} \leq \pi_{2}$ iff $\operatorname{supp}\left(\pi_{1}\right) \supseteq \operatorname{supp}\left(\pi_{2}\right)$ and there is an injection $f: \pi_{2} \rightarrow \pi_{1}$ such that for any block $A \in \pi_{2}$, $f(A) \cap \operatorname{supp}\left(\pi_{2}\right) \subseteq A$, in other words, $f(A)$ overlaps no other block of $\pi_{2}$ than $A$. We give here another equivalent definition:

Lemma 15 Let $\pi_{1}, \pi_{2} \in \Pi^{*}(E)$. Define $\mathbf{A}\left(\pi_{1}, \pi_{2}\right)$ as the set of blocks of $\pi_{1}$ that do not overlap any block of $\pi_{2}$ :

$$
\mathbf{A}\left(\pi_{1}, \pi_{2}\right)=\left\{B \in \pi_{1} \mid B \cap \operatorname{supp}\left(\pi_{2}\right)=\emptyset\right\} ;
$$

then $\mathbf{B}\left(\pi_{1}, \pi_{2}\right)$ as the set of blocks of $\pi_{2}$ such that there is a block of $\pi_{1}$ that overlaps it but does not overlap any other block of $\pi_{2}$ :

$$
\mathbf{B}\left(\pi_{1}, \pi_{2}\right)=\left\{\begin{array}{l|l}
C \in \pi_{2} & \begin{array}{l}
\exists B \in \pi_{1}, \\
\emptyset \neq B \cap \operatorname{supp}\left(\pi_{2}\right) \subseteq C
\end{array}
\end{array} .\right.
$$

Then $\pi_{1} \stackrel{\text { jn }}{\leq} \pi_{2}$ iff $\left|\mathbf{A}\left(\pi_{1}, \pi_{2}\right)\right| \geq\left|\pi_{2} \backslash \mathbf{B}\left(\pi_{1}, \pi_{2}\right)\right|$ and $\operatorname{supp}\left(\pi_{1}\right) \supseteq \operatorname{supp}\left(\pi_{2}\right)$.

Proof Let $\pi_{1} \leq \pi_{2}$, so $\operatorname{supp}\left(\pi_{1}\right) \supseteq \operatorname{supp}\left(\pi_{2}\right)$ and for all $C \in \pi_{2}, f(C) \cap \operatorname{supp}\left(\pi_{2}\right) \subseteq C$; if $\emptyset \neq f(C) \cap \operatorname{supp}\left(\pi_{2}\right)$, then there is $B=f(C) \in \pi_{1}$ with $\emptyset \neq B \cap \operatorname{supp}\left(\pi_{2}\right) \subseteq C$, so $C \in \mathbf{B}\left(\pi_{1}, \pi_{2}\right)$; thus for $C \in \pi_{2} \backslash \mathbf{B}\left(\pi_{1}, \pi_{2}\right)$, we have $f(C) \cap \operatorname{supp}\left(\pi_{2}\right)=\emptyset$, that is, $f(C) \in \mathbf{A}\left(\pi_{1}, \pi_{2}\right)$; as $f$ is an injection, we get $\left|\mathbf{A}\left(\pi_{1}, \pi_{2}\right)\right| \geq\left|\pi_{2} \backslash \mathbf{B}\left(\pi_{1}, \pi_{2}\right)\right|$.

Let $\left|\mathbf{A}\left(\pi_{1}, \pi_{2}\right)\right| \geq\left|\pi_{2} \backslash \mathbf{B}\left(\pi_{1}, \pi_{2}\right)\right|$. There is thus an injection $g: \pi_{2} \backslash \mathbf{B}\left(\pi_{1}, \pi_{2}\right) \rightarrow \mathbf{A}\left(\pi_{1}, \pi_{2}\right)$, so for $C \in \pi_{2} \backslash \mathbf{B}\left(\pi_{1}, \pi_{2}\right)$ we set $f(C)=g(C)$; then $f(C) \cap$ $\operatorname{supp}\left(\pi_{2}\right)=\emptyset \subseteq C$. For $C \in \mathbf{B}\left(\pi_{1}, \pi_{2}\right)$ we choose one $B \in \pi_{1}$ such that $\emptyset \neq B \cap \operatorname{supp}\left(\pi_{2}\right) \subseteq C$, and set $f(C)=B$. Thus we have $f: \pi_{2} \rightarrow \pi_{1}$ such that for all $C \in \pi_{2}, f(C) \cap \operatorname{supp}\left(\pi_{2}\right) \subseteq C$. We now show that $f$ is injective. For two distinct $C_{1}, C_{2} \in \pi_{2} \backslash \mathbf{B}\left(\pi_{1}, \pi_{2}\right)$, $f\left(C_{1}\right)=g\left(C_{1}\right) \neq g\left(C_{2}\right)=f\left(C_{2}\right)$ by definition; for two distinct $C_{1}, C_{2} \in \mathbf{B}\left(\pi_{1}, \pi_{2}\right)$, for $i=1,2$ we have $\emptyset \neq f\left(C_{i}\right) \cap \operatorname{supp}\left(\pi_{2}\right) \subseteq C_{i}$, but as $C_{1}$ and $C_{2}$ are disjoint, the non-empty sets $f\left(C_{1}\right) \cap \operatorname{supp}\left(\pi_{2}\right)$ and $f\left(C_{2}\right) \cap$ $\operatorname{supp}\left(\pi_{2}\right)$ must also be disjoint, thus $f\left(C_{1}\right) \neq f\left(C_{2}\right)$; finally for $C_{1} \in \pi_{2} \backslash \mathbf{B}\left(\pi_{1}, \pi_{2}\right)$ and $C_{2} \in \mathbf{B}\left(\pi_{1}, \pi_{2}\right)$, we have $f\left(C_{1}\right) \in \mathbf{A}\left(\pi_{1}, \pi_{2}\right)$, so $f\left(C_{1}\right) \cap \operatorname{supp}\left(\pi_{2}\right)=\emptyset$, while $f\left(C_{2}\right) \cap \operatorname{supp}\left(\pi_{2}\right) \neq \emptyset$, so $f\left(C_{1}\right) \neq f\left(C_{2}\right)$. Assuming $\operatorname{supp}\left(\pi_{1}\right) \supseteq \operatorname{supp}\left(\pi_{2}\right)$, we deduce that $\pi_{1} \stackrel{\text { jn }}{\leq} \pi_{2}$.

For example in Figure 6, we have $\left|\pi_{2} \backslash \mathbf{B}\left(\pi_{1}, \pi_{2}\right)\right|=$ $\left|\mathbf{A}\left(\pi_{1}, \pi_{2}\right)\right|=3$ and $\left|\mathbf{B}\left(\pi_{1}, \pi_{2}\right)\right|=1$; for the unique block $C \in \mathbf{B}\left(\pi_{1}, \pi_{2}\right)$, there are two blocks of $\pi_{1}$ overlapping $C$ but no other block of $\pi_{2}$, so two possible choices for $f(C)$. The injection $f$ is then built as in the above proof.

Theorem 16 The joining order $\leq$ in $\leq$ is a partial order relation on $\Pi^{*}(E)$. It contains the partial apportioning $\stackrel{\text { pa }}{\leq}$ and linking $\stackrel{\text { In }}{\leq}$ orders, that is, for any $\pi_{1}, \pi_{2} \in \Pi^{*}(E)$, each of $\pi_{1} \stackrel{\text { pa }}{\leq} \pi_{2}$ and $\pi_{1} \stackrel{\text { In }}{\leq} \pi_{2}$ implies $\pi_{1} \stackrel{\text { jn }}{\leq} \pi_{2}$. The greatest and least elements are $\varnothing$ and $\mathbf{0}_{E}$. For any $\pi_{1}, \pi_{2} \in \Pi^{*}(E)$ we have

$$
\pi_{1} \stackrel{\text { a }}{\leq} \pi_{2} \Longleftrightarrow\left[\pi_{1} \stackrel{\text { jn }}{\leq} \pi_{2} \& \operatorname{supp}\left(\pi_{1}\right)=\operatorname{supp}\left(\pi_{2}\right)\right] .
$$

(a) For non-void partial partitions (in $\left.\Pi^{*}(E) \backslash\{\varnothing\}\right)$ : the joining order is generated by apportioning followed by inverse inflating: $\forall \pi_{1}, \pi_{2} \in \Pi^{*}(E) \backslash\{\varnothing\}$,

$$
\pi_{1} \stackrel{\text { jn }}{\leq} \pi_{2} \Longleftrightarrow \exists \pi \in \Pi^{*}(E) \backslash\{\varnothing\}, \pi_{1} \stackrel{\mathrm{a}}{\leq} \pi \stackrel{\mathrm{i}}{\geq} \pi_{2} .
$$


See Figure 6. The maximal elements are the $\mathbf{1}_{\{p\}}$ for $p \in E$. The covering relation is $\stackrel{\mathrm{a}}{\prec} \cup \stackrel{\mathrm{i}}{\succ}$.

Let $E$ be finite. Then $\Pi^{*}(E) \backslash\{\varnothing\}$ ordered by $\stackrel{\text { in }}{\leq}$ is graded by $\left(h_{r}, h_{b}\right)$, equivalently by $\left(-h_{c},-h_{s}\right)$, for $(\stackrel{a}{\prec}$ $, \stackrel{\mathrm{i}}{\succ})$, that is, for any $\pi_{1}, \pi_{2} \in \Pi^{*}(E) \backslash\{\varnothing\}$ we have

$$
\begin{aligned}
& \pi_{1} \stackrel{\text { jn }}{<} \pi_{2} \Longrightarrow {\left[\begin{array}{l}
h_{s}\left(\pi_{1}\right) \geq h_{s}\left(\pi_{2}\right) \& \\
h_{c}\left(\pi_{1}\right) \geq h_{c}\left(\pi_{2}\right) \& \\
\left(h_{s}+h_{c}\right)\left(\pi_{1}\right)>\left(h_{s}+h_{c}\right)\left(\pi_{2}\right)
\end{array}\right], } \\
& \pi_{1} \stackrel{\mathrm{a}}{\prec} \pi_{2} \Longrightarrow\left[\begin{array}{l}
h_{s}\left(\pi_{2}\right)=h_{s}\left(\pi_{1}\right) \& \\
h_{c}\left(\pi_{2}\right)=h_{c}\left(\pi_{1}\right)-1
\end{array}\right], \\
& \pi_{1} \stackrel{\mathrm{i}}{\succ} \pi_{2} \Longrightarrow\left[\begin{array}{l}
h_{c}\left(\pi_{2}\right)=h_{c}\left(\pi_{1}\right) \& \\
h_{s}\left(\pi_{2}\right)=h_{s}\left(\pi_{1}\right)-1
\end{array}\right] .
\end{aligned}
$$

For $\pi \in \Pi^{*}(E) \backslash\{\varnothing\}$, the height of $\pi$ is $h_{r}(\pi)+h_{b}(\pi)$.

(b) For $\varnothing$ : $\varnothing$ covers an element of $\Pi^{*}(E) \backslash\{\varnothing\}$ iff it is maximal, i.e., it is $\mathbf{1}_{\{p\}}$ for some $p \in E$, and then $\mathbf{1}_{\{p\}} \succ^{\mathrm{s}} \varnothing$. When $E$ is finite, the height of $\varnothing$ is $2|E|-1=h_{r}(\varnothing)+h_{b}(\varnothing)-1$.

Proof First we show that the joining order $\stackrel{\text { jn }}{\leq}$ contains the partial apportioning order $\stackrel{\text { pa }}{\leq}$. Indeed, if $\pi_{1} \stackrel{\text { pa }}{\leq} \pi_{2}$, then $\operatorname{supp}\left(\pi_{1}\right) \supseteq \operatorname{supp}\left(\pi_{2}\right)$ and we have an injection $\beta: \pi_{2} \rightarrow \pi_{1}$ that partially apportions $\pi_{1}$ into $\pi_{2}$, that is, for every $A \in \pi_{2}, \beta(A) \subseteq A$, so we get $\beta(A) \cap \operatorname{supp}\left(\pi_{2}\right) \subseteq$ $A \cap \operatorname{supp}\left(\pi_{2}\right)=A$; taking $f=\beta$, we deduce that $\pi_{1} \stackrel{\text { jn }}{\leq} \pi_{2}$. Next we show that the joining order contains the linking order $\stackrel{\ln }{\leq}$. Indeed, if $\pi_{1} \stackrel{\text { In }}{\leq} \pi_{2}$, then $\operatorname{supp}\left(\pi_{1}\right) \supseteq \operatorname{supp}\left(\pi_{2}\right)$ and $\pi_{1} \vdash \pi_{2}$; for any $A \in \pi_{2}$, as $A \subseteq \operatorname{supp}\left(\pi_{2}\right) \subseteq \operatorname{supp}\left(\pi_{1}\right)$, there is at least one $B \in \pi_{1}$ such that $B \gamma A$, so we choose one such $B$ for $f(A)$; as $\pi_{1} \vdash \pi_{2}$ and $f(A) \succ A, f(A)$ does not overlap any other block of $\pi_{2}$, so $f(A) \cap \operatorname{supp}\left(\pi_{2}\right) \subseteq A$; for any two distinct $A_{1}, A_{2} \in \pi_{2}$, we have $f\left(A_{1}\right) \succ A_{1}, f\left(A_{2}\right) \curlyvee A_{2}$, but $f\left(A_{1}\right)$ does not overlap $A_{2}$, so $f\left(A_{1}\right) \neq f\left(A_{2}\right)$, and $f$ is injective. Hence $\pi_{1} \stackrel{\text { jn }}{\leq} \pi_{2}$.

If $\pi_{1} \stackrel{\text { a }}{\leq} \pi_{2}$, then $\operatorname{supp}\left(\pi_{1}\right)=\operatorname{supp}\left(\pi_{2}\right)$ and $\pi_{1} \stackrel{\text { pa }}{\leq} \pi_{2}$, hence $\pi_{1} \stackrel{\text { jn }}{\leq} \pi_{2}$. Conversely, if $\pi_{1} \stackrel{\text { jn }}{\leq} \pi_{2}$ and $\operatorname{supp}\left(\pi_{1}\right)=$ $\operatorname{supp}\left(\pi_{2}\right)$, then for every $A \in \pi_{2}$ we have $f(A)=f(A) \cap$ $\operatorname{supp}\left(\pi_{1}\right)=f(A) \cap \operatorname{supp}\left(\pi_{2}\right) \subseteq A$, so $\pi_{1} \Subset \pi_{2}$, hence $\pi_{1} \stackrel{\text { a }}{\leq} \pi_{2}$. Thus (16) holds.

We now show that $\underset{\leq}{\leq}$ is a partial order relation on $\Pi^{*}(E)$. First $\leq$ is reflexive: $\operatorname{supp}(\pi) \subseteq \operatorname{supp}(\pi)$ and taking for $f$ the identity on $\pi$, for all $A \in \pi$ we have $f(A) \cap \operatorname{supp}(\pi)=A \subseteq A$. Next, it is antisymmetric: if $\pi_{1} \stackrel{\text { jn }}{\leq} \pi_{2}$ and $\pi_{2} \stackrel{\text { jn }}{\leq} \pi_{1}$, then by double inclusion we get $\operatorname{supp}\left(\pi_{1}\right)=\operatorname{supp}\left(\pi_{2}\right)$, and (16) gives $\pi_{1} \stackrel{\text { a }}{\leq} \pi_{2}$ and $\pi_{2} \stackrel{\text { a }}{\leq} \pi_{1}$, hence $\pi_{1}=\pi_{2}$. Finally, it is transitive: let $\pi_{0} \stackrel{\text { jn }}{\leq} \pi_{1} \stackrel{\text { jn }}{\leq} \pi_{2}$, so $\operatorname{supp}\left(\pi_{0}\right) \supseteq \operatorname{supp}\left(\pi_{1}\right) \supseteq \operatorname{supp}\left(\pi_{2}\right)$, and there are two injections $f_{2}: \pi_{2} \rightarrow \pi_{1}$ and $f_{1}: \pi_{1} \rightarrow \pi_{0}$ such that for any $A \in \pi_{2}, f_{2}(A) \cap \operatorname{supp}\left(\pi_{2}\right) \subseteq A$ and for any $B \in \pi_{1}, f_{1}(B) \cap \operatorname{supp}\left(\pi_{1}\right) \subseteq B$; thus $f_{1} f_{2}$ is an injection $\pi_{2} \rightarrow \pi_{0}$ and for any $A \in \pi_{2}, f_{1} f_{2}(A) \cap \operatorname{supp}\left(\pi_{1}\right) \subseteq$ $f_{2}(A)$, so

$$
\begin{gathered}
f_{1} f_{2}(A) \cap \operatorname{supp}\left(\pi_{2}\right)=f_{1} f_{2}(A) \cap\left(\operatorname{supp}\left(\pi_{1}\right) \cap \operatorname{supp}\left(\pi_{2}\right)\right) \\
=\left(f_{1} f_{2}(A) \cap \operatorname{supp}\left(\pi_{1}\right)\right) \cap \operatorname{supp}\left(\pi_{2}\right) \\
\subseteq f_{2}(A) \cap \operatorname{supp}\left(\pi_{2}\right) \subseteq A,
\end{gathered}
$$

and we deduce that $\pi_{0} \stackrel{\text { jn }}{\leq} \pi_{2}$.

Since the joining order contains the linking order, it inherits from it its greatest and least elements $\varnothing$ and $\mathbf{0}_{E}$. The same argument as in the proof of Theorem 9 shows that the maximal elements of $\Pi^{*}(E) \backslash\{\varnothing\}$ are the $\mathbf{1}_{\{p\}}$ for $p \in E$.

If $\pi_{1} \stackrel{\text { a }}{\leq} \pi \stackrel{\text { i }}{\geq} \pi_{2}$, then $\pi_{1} \stackrel{\text { pa }}{\leq} \pi \stackrel{\text { In }}{\leq} \pi_{2}$, so $\pi_{1} \stackrel{\text { jn }}{\leq} \pi \stackrel{\text { jn }}{\leq} \pi_{2}$, thus $\pi_{1} \stackrel{\text { jn }}{\leq} \pi_{2}$. Conversely, let $\pi_{1}, \pi_{2} \in \Pi^{*}(E) \backslash\{\emptyset\}$ such that $\pi_{1} \stackrel{\text { jn }}{\leq} \pi_{2}: \operatorname{supp}\left(\pi_{1}\right) \supseteq \operatorname{supp}\left(\pi_{2}\right)$ and there is an injection $f: \pi_{2} \rightarrow \pi_{1}$ such that for all $A \in \pi_{2}$, $f(A) \cap \operatorname{supp}\left(\pi_{2}\right) \subseteq A$. For any two distinct $A_{1}, A_{2} \in \pi_{2}$, $A_{1} \cap A_{2}=\emptyset$, and as $f$ is injective, $f\left(A_{1}\right) \neq f\left(A_{2}\right)$, so $f\left(A_{1}\right) \cap f\left(A_{2}\right)=\emptyset$; now

$$
\begin{aligned}
& f\left(A_{1}\right) \cap A_{2}=f\left(A_{1}\right) \cap\left(\operatorname{supp}\left(\pi_{2}\right) \cap A_{2}\right) \\
= & \left(f\left(A_{1}\right) \cap \operatorname{supp}\left(\pi_{2}\right)\right) \cap A_{2} \subseteq A_{1} \cap A_{2}=\emptyset,
\end{aligned}
$$

and similarly $f\left(A_{2}\right) \cap A_{1}=\emptyset$; therefore

$$
\begin{gathered}
\left(A_{1} \cup f\left(A_{1}\right)\right) \cap\left(A_{2} \cup f\left(A_{2}\right)\right)= \\
\left(A_{1} \cap A_{2}\right) \cup\left(f\left(A_{1}\right) \cap A_{2}\right) \\
\cup\left(A_{1} \cap f\left(A_{2}\right)\right) \cup\left(f\left(A_{1}\right) \cap f\left(A_{2}\right)\right)=\emptyset .
\end{gathered}
$$

Thus the $A \cup f(A)$ for $A \in \pi_{2}$ are pairwise disjoint; moreover, as $A \in \pi_{2}$ and $f(A) \in \pi_{1}$, we get $A \subseteq$ $\operatorname{supp}\left(\pi_{2}\right) \subseteq \operatorname{supp}\left(\pi_{1}\right)$ and $f(A) \subseteq \operatorname{supp}\left(\pi_{1}\right)$, so $A \cup$ $f(A) \subseteq \operatorname{supp}\left(\pi_{1}\right)$. Let $Z=\operatorname{supp}\left(\pi_{1}\right) \backslash \bigcup_{A \in \pi_{2}}(A \cup f(A))$ and choose any map $g: Z \rightarrow \pi_{2}$. Then the $g^{-1}(A)=$ $\{p \in Z \mid g(p)=A\}$ for $A \in \pi_{2}$ are pairwise disjoint, and as $Z$ is disjoint from all $A \cup f(A)$, each $g^{-1}\left(A_{0}\right)$ $\left(A_{0} \in \pi_{2}\right)$ is disjoint from all $A \cup f(A)\left(A \in \pi_{2}\right)$. Hence $\pi=\left\{A \cup f(A) \cup g^{-1}(A) \mid A \in \pi_{2}\right\}$ is a partial partition. See Figure 16. We have

$$
\operatorname{supp}(\pi)=\bigcup_{A \in \pi_{2}}(A \cup f(A)) \cup Z=\operatorname{supp}\left(\pi_{1}\right) .
$$

Now each block of $\pi$ is some $A \cup f(A) \cup g^{-1}(A), A \in \pi_{2}$, and it contains $f(A) \in \pi_{1}$; thus $\pi_{1} \Subset \pi$, hence $\pi_{1} \stackrel{\text { a }}{\leq} \pi$. Now for any $A \in \pi_{2}, A \subseteq A \cup f(A) \cup g^{-1}(A) \in \pi$ and every $A \cup f(A) \cup g^{-1}(A) \in \pi$ contains $A$ but no other 

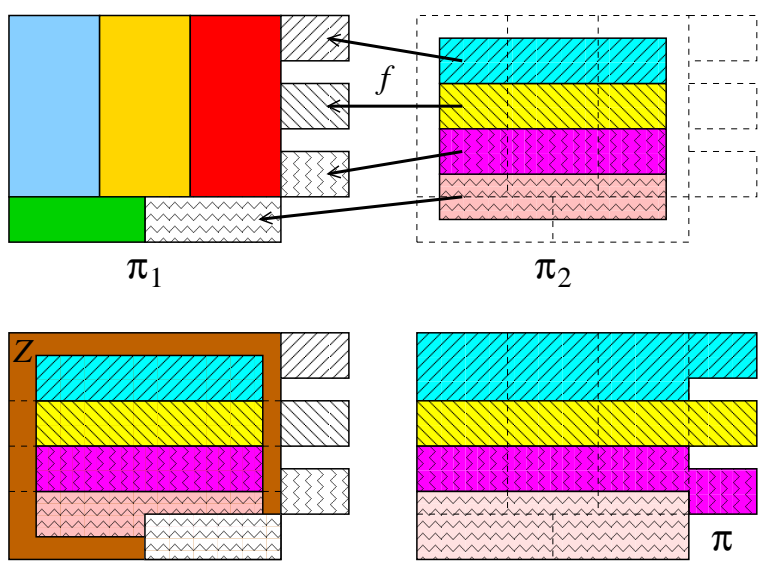

Fig. 16 Top row: from Figure $6, \pi_{1}, \pi_{2}$ and the injection $f: \pi_{2} \rightarrow \pi_{1}$ (indicated by arrows); for each $A \in \pi_{2}, f(A)$ is shown with the same hatching as $A$ (but without colour), while other blocks of $\pi_{1}$ are shown without hatching. Bottom row, left: we show the $A$ and $f(A)$ for $A \in \pi_{2}$, and the portion $Z$ of $\operatorname{supp}\left(\pi_{1}\right)$ not covered by them; the dotted lines indicate how $Z$ will be apportioned. Bottom row, right: apportioning $Z$ to the $A \cup f(A), A \in \pi_{2}$, we obtain $\pi$.

block $A^{\prime} \in \pi_{2}$ (because $A^{\prime}$ is disjoint from $A, f(A)$ and $\left.g^{-1}(A)\right)$, hence $\pi_{2} \stackrel{i}{\leq} \pi$.

Let $\stackrel{\text { jn }}{\prec}$ be the covering relation for $\stackrel{\text { jn }}{\leq}$. As in the proof of Theorem 9, we apply Lemmas 1 and 2 with $\Pi^{*}(E) \backslash$ $\{\varnothing\}, \stackrel{\text { jn }}{\leq}, \stackrel{\text { jn }}{\prec}, \stackrel{\text { a }}{\leq}, \stackrel{\text { a }}{\prec}, \stackrel{\text { i }}{\geq}, \stackrel{i}{\succ}, \mathcal{P}(E), \supseteq$ and supp standing for $P, \leq, \prec, \stackrel{\alpha}{\leq}, \stackrel{\alpha}{\prec}, \stackrel{\beta}{\leq}, \stackrel{\beta}{\prec}, Q$, $\pi_{1}, \pi_{2} \in \Pi^{*}(E) \backslash\{\varnothing\}:$ (i) if $\pi_{1} \stackrel{\text { jn }}{\prec} \pi_{2}$, then $\pi_{1} \stackrel{\text { a }}{\prec} \pi_{2}$ or $\pi_{1} \stackrel{\mathrm{i}}{\succ}^{2} \pi_{2}$; (ii) if $\pi_{1} \stackrel{\text { a }}{\prec} \pi_{2}$, then $\pi_{1} \stackrel{\text { jn }}{\prec} \pi_{2}$. Let us now show that (iii) if $\pi_{1} \stackrel{\text { i }}{\succ} \pi_{2}$, then $\pi_{1} \stackrel{\text { jn }}{\prec} \pi_{2}$. Let $\pi_{1} \stackrel{\text { i }}{\succ}$ $\pi_{2}$, where $\pi_{2}$ is obtained by removing a point $p$ from a block of $\pi_{1}$ that also contains another point; then $\pi_{2}=\left\{C \backslash\{p\} \mid C \in \pi_{1}\right\}$. As in the proof of Theorem 9, a chain $\pi_{1} \leq \cdots \stackrel{\text { jn }}{\leq} \ldots \pi_{2}$ reduces to $\pi_{1} \stackrel{\text { a }}{\leq} \pi_{3} \stackrel{\text { i }}{>} \pi_{4} \stackrel{\text { a }}{\leq} \pi_{2}$, where $\pi_{4}$ is obtained by removing the point $p$ from a block of $\pi_{3}$, thus $\pi_{4}=\left\{C \backslash\{p\} \mid C \in \pi_{3}\right\}$. A block of $\pi_{2}$ is of the form $C \backslash\{p\}$ for $C \in \pi_{1} ;$ as $\pi_{4} \Subset \pi_{2}, C \backslash\{p\}$ contains a block of $\pi_{4}$, which is of the form $B \backslash\{p\}$ for $B \in \pi_{3}$; as $\pi_{1} \Subset \pi_{3}, B$ contains a block $A \in \pi_{1}$; thus $A \backslash\{p\} \subseteq B \backslash\{p\} \subseteq C \backslash\{p\}$, where $A, C \in \pi_{1}$, and as $A \backslash\{p\}, C \backslash\{p\} \neq \emptyset$, we have $A=C$, hence $B \backslash\{p\}=A \backslash\{p\}=C \backslash\{p\}$, with $C \backslash\{p\} \in \pi_{2}$ and $B \backslash\{p\} \in \pi_{4}$. Thus each block of $\pi_{2}$ is equal to a block of $\pi_{4}$, so $\pi_{2} \subseteq \pi_{4}$, and as $\pi_{4} \stackrel{\text { a }}{\leq} \pi_{2}$, we deduce from [16] that $\pi_{4}=\pi_{2}$. Since $C=A \subseteq B$ and $C \backslash\{p\}=B \backslash\{p\}$, we have $C=B$ or $B=C \cup\{p\}$. Suppose that for some $C \in \pi_{1}$ we have $B=C \cup\{p\} \in \pi_{3}$; thus the block of $\pi_{1}$ containing $p$ has been apportioned, with the part $\{p\}$ merged with $C$; as this apportioned block contains at least another point, there is another part $D$ merged with another block $C^{\prime} \in \pi_{1}$, so we have $B^{\prime} \in \pi_{3}$ with $C^{\prime} \cup D \subseteq B^{\prime}, D \neq \emptyset$ and $p \notin D$; then the above argument with $C^{\prime}$ instead of $C$ will lead to $B^{\prime}=C^{\prime}$ or $B^{\prime}=C^{\prime} \cup\{p\}$, a contradiction. Thus $C=B$ for all $C \in \pi_{1}$, so every block of $\pi_{1}$ is equal to a block of $\pi_{3}$, hence $\pi_{1} \subseteq \pi_{3}$, and as $\pi_{1} \stackrel{\text { a }}{\leq} \pi_{3}$, we deduce from [16] that $\pi_{1}=\pi_{3}$. Therefore $\pi_{1} \stackrel{\text { jn }}{\prec} \pi_{2}$. We have thus shown that the covering relation for $\leq$ on $\Pi^{*}(E) \backslash\{\varnothing\}$ is $\stackrel{a}{\prec} \cup \stackrel{\text { i }}{\succ}$.

The same argument as in the proof of Theorem 9 shows that for $\pi \in \Pi^{*}(E) \backslash\{\varnothing\}, \pi \stackrel{\text { jn }}{\prec} \varnothing$ iff $\pi$ is a maximal element of $\Pi^{*}(E) \backslash\{\varnothing\}$, that is, $\mathbf{1}_{\{p\}}$ for some

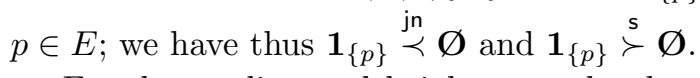

For the grading and height, we take the argument in the proof of Theorem 9 , where we replace $\stackrel{\mathrm{m}}{\leq}, \stackrel{\mathrm{m}}{<}$ and $\stackrel{\mathrm{m}}{\prec}$ by $\stackrel{\text { a }}{\leq}, \stackrel{\text { a }}{<}$ and $\stackrel{\text { a }}{\prec}$ respectively.

Now the partial apportioning order $\stackrel{\text { pa }}{\leq}$ contains the

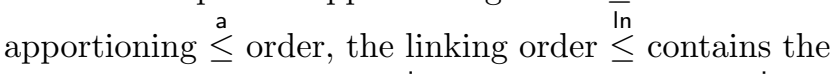
inverse inclusion-inflating $\supseteq$ and inverse inflating $\geq$ orders, and both contain the merging $\stackrel{m}{\leq}$, inverse inclusion $\supseteq$, regional $\stackrel{\mathrm{rg}}{\leq}$ and partial merging order $\stackrel{\mathrm{pm}}{\leq}$. It follows that the joining order $\leq$ contains also the merging $\stackrel{m}{\leq}$, apportioning $\stackrel{a}{\leq}$, inverse inflating $\stackrel{i}{\geq}$, inverse inclusion $\supseteq$, inverse inclusion-inflating $\supseteq$ pm merging $\leq$ orders.

\section{Discussion and conclusion}

\subsection{Review of our orders on partial partitions}

The standard order $\leq$ on $\Pi^{*}(E)$ was first considered by Draškovičová $[6,7]$ and further studied in [12,13]; together with the identity, it remained for a long time the only known partial order relation on $\Pi^{*}(E)$. Then Serra introduced the building order $\Subset[20,21]$. A systematic study of orders on partial partitions was initiated by the author: 5 new orders were introduced in [15] and 3 more in [16]. Among them, 4 are simple: the inclusion $\subseteq$, inflating $\stackrel{\mathrm{i}}{\leq}$, merging $\stackrel{\mathrm{m}}{\leq}$ and apportioning $\stackrel{\mathrm{a}}{\leq}$ orders, where apportioning $\stackrel{\text { a }}{\leq}$ is a generalization of merging $\stackrel{m}{\leq}$. The other orders are compound, each one being generated by combining two simple orders; they are the standard order $\leq$ and the 4 other new orders: the inclusioninflating $\stackrel{\mathrm{i}}{\subseteq}$, merging-inflating $\stackrel{\mathrm{mi}}{\leq}$, apportioning-inflating 
$\stackrel{\text { ai }}{\leq}$ and extended $\stackrel{\text { e }}{\leq}$ orders. We give below their generation by combining simple orders:

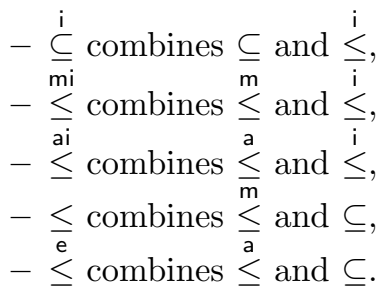

Note that the standard $\leq$ and extended $\stackrel{e}{\leq}$ also contain the inflating order $\stackrel{i}{\leq}$. Indeed, in item 1 of Proposition 10 of [15] we showed that inflating blocks can be achieved by creating then merging blocks: $\forall \pi_{1}, \pi_{2} \in \Pi^{*}(E)$,

$$
\pi_{1} \stackrel{\mathrm{i}}{\leq} \pi_{2} \Longrightarrow \exists \pi \in \Pi^{*}(E), \pi_{1} \subseteq \pi \stackrel{\mathrm{m}}{\leq} \pi_{2}
$$

The standard order and the 8 new orders of $[15,16]$ are graded; the 4 simple orders have a simple grading, the 5 compound ones have a double grading. For all these orders, growing a partial partition either preserves or increases its support, while existing blocks can grow by merging or inflating.

These orders of $[15,16]$ are relevant in image filtering, where one aims at producing large homogeneous zones in images, and also in modeling image segmentation based on region growing (such as the watershed), as well as hierarchies of segmentations $[12,15,16]$.

In this paper we have introduced several new orders on $\Pi^{*}(E)$, where in a growing partial partition, both the support and the number of blocks can only decrease or remain equal. They all contain the regional order $\stackrel{\text { rg }}{\leq}$ generated by the merging $\stackrel{m}{\leq}$ and inverse inclusion $\supseteq$ orders, so merging or removing blocks increases the partial partition. Thus they share the latter's least element $\mathbf{0}_{E}$ and greatest element $\varnothing$.

The regional $\stackrel{\mathrm{rg}}{\leq}$, partial merging $\stackrel{\mathrm{pm}}{\leq}$, and partial apportioning $\leq$ orders are simple, and when $E$ is finite they all share the same simple grading by $h_{r}$ (equivalently, by $-h_{c}$ ), which counts the decrease of the number of blocks: the growth of a partial partition necessarily decreases the number of blocks. Although the regional order $\stackrel{\text { rg }}{\leq}$ combines two operations, namely block removal and block merging, the two operations cannot be counted separately; as explained above, removing $k$ blocks can be obtained by $k-1$ successive block mergings followed by the removal of the merged block. In fact, the regional and partial merging orders are included in the partial apportioning order, so the operations of merging, apportioning, removing or partial merging of blocks are particular cases of partial apportioning.

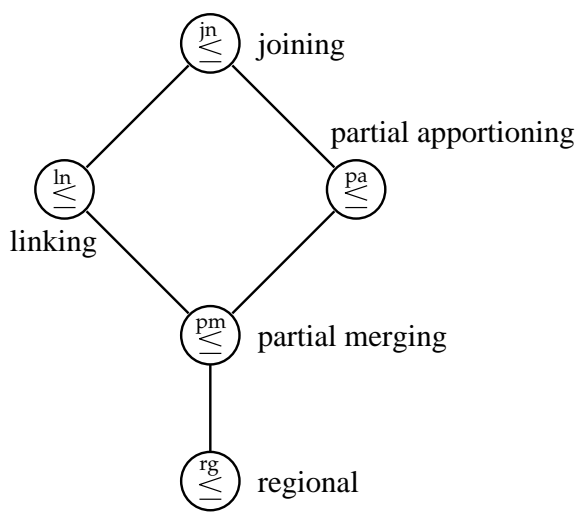

Fig. 17 Hasse diagram of the 5 new orders.

These simple orders are incompatible with block deflating: if $\pi_{1} \geq \pi_{2}$ and $\pi_{1} \stackrel{\text { pa }}{\leq} \pi_{2}$, then $\pi_{1}=\pi_{2}$; indeed, $\pi_{1} \stackrel{\text { i }}{\geq} \pi_{2} \Rightarrow \pi_{1} \ni \pi_{2}[15], \pi_{1} \stackrel{\text { pa }}{\leq} \pi_{2} \Rightarrow \pi_{1} \Subset \pi_{2}(13)$ and the antisymetry of $\Subset$ leads to the equality. More intuitively, when $E$ is finite, deflating blocks decreases the support of the partial partition without changing the number $h_{c}(\pi)$ of blocks.

We have two compound orders containing the inverse inflating order $\stackrel{i}{\geq}$, the linking $\stackrel{\text { In }}{\leq}$ and joining $\stackrel{\text { jn }}{\leq}$ orders, they combine $\geq$ with respectively the merging $\stackrel{m}{\leq}$ and apportioning $\stackrel{\text { a }}{\leq}$ order. They also contain the inverse inclusion order $\supseteq$. Indeed, in item 3 of Proposition 10 of [15] we showed that for non-void partial partitions, removing some - but not all-blocks can be achieved by merging blocks, then deflating blocks: $\forall \pi_{1}, \pi_{2} \in \Pi^{*}(E) \backslash\{\varnothing\}$,

$$
\pi_{1} \supseteq \pi_{2} \Longrightarrow \exists \pi \in \Pi^{*}(E), \pi_{1} \stackrel{\mathrm{m}}{\leq} \pi \stackrel{\mathrm{i}}{\geq} \pi_{2} .
$$

On the other hand, the empty partial partition $\varnothing$ is not obtained by combining block mergings and deflations, it is necessary to remove a last block. Thus these orders are effectively compound on $\Pi^{*}(E) \backslash\{\varnothing\}$. When $E$ is finite, these two orders restricted to $\Pi^{*}(E) \backslash\{\varnothing\}$ have a double grading: $h_{r}$ (equivalently, $-h_{c}$ ) counts the decrease of the number of blocks, obtained by block mergings or apportionings, and $h_{b}$ (equivalently, $-h_{s}$ ) counts the decrease of the size of the support, obtained by block deflations.

The Hasse diagram of the inclusion relation between the regional, linking, partial apportioning, partial merging and joining orders is shown in Figure 17.

In [16] it was shown that the building order is generated by composing the inverse inclusion $\supseteq$ and inflating $\leq$ orders; it also contains the merging order. Indeed, in item 2 of Proposition 10 of [15] we showed that merging blocks can be achieved by removing then inflating 
blocks: $\forall \pi_{1}, \pi_{2} \in \Pi^{*}(E)$,

$$
\pi_{1} \stackrel{\mathrm{m}}{\leq} \pi_{2} \Longrightarrow \exists \pi \in \Pi^{*}(E), \pi_{1} \supseteq \pi \stackrel{\mathrm{i}}{\leq} \pi_{2} .
$$

The building order is not graded and does not satisfy the Jordan-Dedekind chain condition. Moreover when $E$ is finite, its covering chains take a strange form, see the Appendix in [16]. We think that this is related to the fact that this order does not involve any condition on the support, which can alternatively grow and shrink.

In the same way as $[15,16]$ considerered all combinations of the basic simple orders $\subseteq, \stackrel{i}{\leq}, \stackrel{m}{\leq}$ and $\stackrel{a}{\leq}$, one can consider the combinations of one with the inverse of another (excluding the combination of apportioning $\stackrel{\text { a }}{\leq}$ with the inverse $\stackrel{m}{\geq}$ of merging, since $\stackrel{m}{\leq}$ is included in $\stackrel{a}{\leq}$ ). We have the following combinations of one basic order with the inverse of another:

$-\Subset$ combines $\stackrel{i}{\leq}$ and $\supseteq$,

$-\underset{\ln }{\mathrm{rg}}$ combines $\underset{\mathrm{m}}{\leq}$ and $\underset{\mathrm{i}}{\mathrm{m}}$,

$-\stackrel{\ln }{\leq}$ combines $\stackrel{\mathrm{m}}{\leq}$ and $\underset{\mathrm{j}}{\geq}$,

$-\stackrel{\text { jn }}{\leq}$ combines $\stackrel{\text { a }}{\leq}$ and $\stackrel{i}{\geq}$.

There remains one further combination, that of $\stackrel{a}{\leq}$ and $\supseteq$. We have checked that this order (containing the regional order) consists of either apportionings or partial apportionings where among the partially apportioned blocks there is one that is completely removed. Thus this order is not really meaningful in practice, since we can consider instead the partial apportioning order $\stackrel{\text { pa }}{\leq}$, which is both wider and conceptually simpler.

Similarly, the joining order $\underset{\mathrm{a}}{\leq}$, which is generated by combining the apportioning $\stackrel{a}{\leq}$ and inverse inflating $\geq$ orders, has a counter-intuitive definition in terms of blocks. Is there a wider and conceptually simpler order? Note that any order strictly containing $\leq$ wn would have to involve a new operation on partial partitions.

On the other hand, the regional $\stackrel{\text { rg }}{\leq}$, linking $\stackrel{\text { In }}{\leq}$ and partial apportioning $\leq$ orders are conceptually simple and seem also to have some practical uses. We have shown that in a hierarchy of partitions, the partial partition of block boundaries increases for the regional order. We have hinted that the linking order can be used to describe the simplification of segmentation markers, or applied in geographic information processing. We have also indicated that the justification of apportioning by the need to eliminate "parasitic segmentation classes" lying along transitions [20,21] is also valid for the partial apportioning order if the segmentation gives a partial partition with a background.
Finally, we are uncertain about the possible uses of the partial merging order $\stackrel{\mathrm{pm}}{\leq}$; it was introduced as the intersection of the linking and partial apportioning orders. More applications are needed for the new orders described in this paper.

Partial partitions are more flexible than partitions, both for practical uses [12] in image filtering and segmentation, and in terms of possible orders [15]. We saw in $[15,16]$ that restricting orders to partial partitions with a fixed support, for instance to partitions of $E$, then

- the inclusion $\subseteq$, inflating $\stackrel{i}{\leq}$ and inclusion-inflating $\stackrel{\mathrm{i}}{\subseteq}$ orders reduce to the identity $=$;

- the standard $\leq$ and merging-inflating $\leq$ mi orders reduce to the merging order $\stackrel{m}{\leq}$;

- the building $\Subset$, apportioning-inflating $\stackrel{\text { ai }}{\leq}$ and extended $\stackrel{e}{\leq}$ orders reduce to the apportioning order $\stackrel{a}{\leq}$

Here we see that by restricting partial partitions to a fixed support,

- by $(9)$, the regional $\stackrel{\mathrm{rg}}{\leq}$, partial merging $\stackrel{\mathrm{pm}}{\leq}$ and linking $\stackrel{\text { In }}{\leq}$ orders reduce to the merging order $\stackrel{m}{\leq}$;

- by (16), the partial apportioning $\leq$ and joining orders $\stackrel{\text { jn }}{\leq}$ reduce to the apportioning order $\stackrel{\text { a }}{\leq}$.

In other words, for partial partitions with a fixed support, the only orders that we get are the identity $=$, merging $\stackrel{m}{\leq}$ and apportioning $\stackrel{\text { a }}{\leq}$ orders.

\subsection{Conclusion and perspectives}

This paper ends our study of orders on partial partitions relevant for image analysis. The previous two papers $[15,16]$ introduced 8 new orders relevant for image filtering and segmentation. Here we introduced 5 new ones, motivated by image simplification or reduction: the regional, linking, partial apportioning, partial merging and joining orders; the inclusion relation between them is shown in Figure 17. We did not explore further combinations, such as that of the apportioning order with the regional or partial merging orders, they give only some special cases of partial apportioning.

The most important contribution of this paper is the regional order, which is isomorphic to the refinement order for partitions on the extended space in which a background marker point has been added. It is also involved in hierarchies of partitions. We think that the 
linking and partial apportioning orders should also be useful.

An interesting question is whether there exist other basic operations for simplifying partial partitions than the ones we used here: merging, partial merging, apportioning, partial apportioning, removing and deflating blocks. A related question is whether the joining order is included in a larger order that is conceptually simpler, using thus more general operations.

In [12] we discussed "connective" image segmentation in the framework of the two complete lattices of partitions for the refinement order and partial partitions for the standard order, and we indicated that the set of "good" (partial) partitions (w.r.t. to a criterion) had to be closed under supremum, so the "best" choice was the supremum of all "good" ones. In [14], we discussed the idea in the case where the order does not constitute a lattice, then the "best" partial partition must be chosen among all "good" ones as one that is maximal for the order. The same logic should be applied here: if we have a criterion for partial partitions associated to an image, the "best" choice for a reduced or simplified image should be one whose associated partial partition should be, among all those satisfying the criterion, one that is maximal for the order.

Several criteria for image segmentation were given in [17], they were "connective" in the sense that the set of corresponding (partial) partitions is stable under supremum. We also saw in [14] that some practical issues (such as transforming a partial partition by acting separately on each block, or by a succession of several operations) can constrain the order to be used. A possible axis of research is the study of criteria for image simplification or reduction having interesting properties in relation to some of the orders studied here, or the restrictions on the orders imposed by practical image processing requirements.

\section{A More about single overlap}

In [15] we indicated that the singularity relation satisfies the following:

$$
\begin{aligned}
& \forall \pi_{1}, \pi_{2}, \pi_{3}, \pi_{4} \in \Pi^{*}(E), \\
& {\left[\pi_{1} \Leftarrow \pi_{2} \& \pi_{3} \subseteq \pi_{1} \& \pi_{4} \subseteq \pi_{2}\right] \Longrightarrow \pi_{3} \Leftarrow \pi_{4} .}
\end{aligned}
$$

We have the counterpart of it for single overlap:

$$
\begin{aligned}
& \forall \pi_{1}, \pi_{2}, \pi_{3}, \pi_{4} \in \Pi^{*}(E), \\
& {\left[\pi_{1} \vdash \pi_{2} \text { and } \pi_{3} \subseteq \pi_{1} \text { and } \pi_{4} \subseteq \pi_{2}\right] \Longrightarrow \pi_{3} \vdash \pi_{4} .}
\end{aligned}
$$

In particular, single overlap is preserved by restriction: for any $A \in \mathcal{P}(E), \pi_{1} \vdash \pi_{2} \Rightarrow \pi_{1} \cap \mathcal{P}(A) \vdash \pi_{2} \cap \mathcal{P}(A)$. Now the following complements Proposition 7:
Proposition 17 For any $\pi_{1}, \pi_{2} \in \Pi^{*}(E)$,

$$
\begin{array}{lrl}
\forall \pi_{0} \in \Pi^{*}(E), & \pi_{0} \leq \pi_{1} \vdash \pi_{2} & \Longrightarrow \pi_{0} \vdash \pi_{2}, \\
\forall A \in \mathcal{P}(E), & \pi_{1} \vdash \pi_{2} \Longrightarrow \pi_{1} \vdash \pi_{2} \wedge \mathbf{1}_{A}, \\
\forall A \supseteq \operatorname{supp}\left(\pi_{1}\right), & \pi_{1} \vdash \pi_{2} \Longleftrightarrow \pi_{1} \vdash \pi_{2} \wedge \mathbf{1}_{A} .
\end{array}
$$

In particular, single overlap is preserved by truncation: for any $A \in \mathcal{P}(E), \pi_{1} \vdash \pi_{2} \Rightarrow \pi_{1} \wedge \mathbf{1}_{A} \vdash \pi_{2} \wedge \mathbf{1}_{A}$.

Proof Let $\pi_{1} \vdash \pi_{2}$, that is (by Proposition 7), $\pi_{1} \wedge \mathbf{1}_{\text {supp }\left(\pi_{2}\right)} \leq$ $\pi_{2}$. Then for $\pi_{0} \in \Pi^{*}(E)$ such that $\pi_{0} \leq \pi_{1}$, we get $\pi_{0} \wedge$ $\mathbf{1}_{\text {supp }\left(\pi_{2}\right)} \leq \pi_{1} \wedge \mathbf{1}_{\text {supp }\left(\pi_{2}\right)} \leq \pi_{2}$, that is, $\pi_{0} \vdash \pi_{2}$.

For $A \in \mathcal{P}(E)$ we have $\operatorname{supp}\left(\pi_{2} \wedge \mathbf{1}_{A}\right)=\operatorname{supp}\left(\pi_{2}\right) \cap A$, so $\mathbf{1}_{\text {supp }\left(\pi_{2} \wedge 1_{A}\right)}=\mathbf{1}_{\operatorname{supp}\left(\pi_{2}\right) \cap A}=\mathbf{1}_{\operatorname{supp}\left(\pi_{2}\right)} \wedge \mathbf{1}_{A}$, hence

$$
\pi_{1} \wedge \mathbf{1}_{\text {supp }\left(\pi_{2} \wedge \mathbf{1}_{A}\right)}=\left(\pi_{1} \wedge \mathbf{1}_{\operatorname{supp}\left(\pi_{2}\right)}\right) \wedge \mathbf{1}_{A} \leq \pi_{2} \wedge \mathbf{1}_{A},
$$

thus $\pi_{1} \vdash \pi_{2} \wedge \mathbf{1}_{A}$. Combining both results with $\pi_{0}=\pi_{1} \wedge \mathbf{1}_{A}$, we get $\pi_{1} \vdash \pi_{2} \Rightarrow \pi_{1} \wedge \mathbf{1}_{A} \vdash \pi_{2} \Rightarrow \pi_{1} \wedge \mathbf{1}_{A} \vdash \pi_{2} \wedge \mathbf{1}_{A}$ : single overlap is preserved by truncation.

Suppose finally that $A \supseteq \operatorname{supp}\left(\pi_{1}\right)$; then $\pi_{1} \leq \mathbf{1}_{A}$; now let $\pi_{1} \vdash \pi_{2} \wedge \mathbf{1}_{A}$, that is, $\pi_{1} \wedge \mathbf{1}_{\operatorname{supp}\left(\pi_{2} \wedge \mathbf{1}_{A}\right)} \leq \pi_{2} \wedge \mathbf{1}_{A}$; we saw above that $\mathbf{1}_{\operatorname{supp}\left(\pi_{2} \wedge \mathbf{1}_{A}\right)}=\mathbf{1}_{\operatorname{supp}\left(\pi_{2}\right)} \wedge \mathbf{1}_{A}$; hence we get

$$
\begin{gathered}
\pi_{1} \wedge \mathbf{1}_{\text {supp }\left(\pi_{2}\right)}=\left(\pi_{1} \wedge \mathbf{1}_{A}\right) \wedge \mathbf{1}_{\text {supp }\left(\pi_{2}\right)}=\pi_{1} \wedge\left(\mathbf{1}_{\text {supp }\left(\pi_{2}\right)} \wedge \mathbf{1}_{A}\right) \\
=\pi_{1} \wedge \mathbf{1}_{\text {supp }\left(\pi_{2} \wedge 1_{A}\right) \leq \pi_{2} \wedge \mathbf{1}_{A} \leq \pi_{2}}
\end{gathered}
$$

therefore $\pi_{1} \vdash \pi_{2}$.

As a counterpart of single overlap, we could consider the relation given by "every block of $\pi_{1}$ overlaps (at least) one block of $\pi_{2}$ ", which is equivalent both to $\pi_{1} \wedge \mathbf{1}_{\text {supp }\left(\pi_{2}\right)} \Subset \pi_{1}$ and to $\pi_{1} \wedge \pi_{2} \Subset \pi_{1}$. However it seems that this relation is too general to produce a new partial order relation by intersecting it with other relations.

\section{References}

1. Adams, R., Bischof, L.: Seeded region growing. IEEE Transactions on Pattern Analysis and Machine Intelligence 16(6), 641-647 (1994)

2. Attali, D., Boissonnat, J.D., Edelsbrunner, H.: Stability and computation of the medial axis - a state-ofthe-art report. In: T. Möller, B. Hamann, R.D. Russell (eds.) Mathematical Foundations of Scientific Visualization, Computer Graphics, and Massive Data Exploration, p. 109125. Springer-Verlag (2009)

3. Bell, E.T.: Exponential numbers. The American Mathematical Monthly 41(7), 411-419 (1934)

4. Bertrand, G., Couprie, M.: Isthmus based parallel and symmetric 3D thinning algorithms. Graphical Models 80, 1-15 (2015). DOI 10.1016/j.gmod.2015.05.001

5. Birkhoff, G.: Lattice Theory, American Mathematical Society Colloquium Publications, vol. 25, 8th printing, 3rd edn. American Mathematical Society (1995)

6. Dras̆kovičová, H.: The lattice of partitions in a set. Acta Fac. Rerum Natur. Univ. Comenian. Math. Publ. 24, 3765 (1970)

7. Draškovičová, H.: Congruence relations on the lattice of partitions in a set. Matematický C̆asopis (Slovenska Akademia Vied) 21, 141-153 (1971)

8. Grätzer, G.: General Lattice Theory. Birkhäuser, 2nd edition, Basel (2003) 
9. Meyer, F., Najman, L.: Segmentation, minimum spanning tree and hierarchies. In: L. Najman, H. Talbot (eds.) Mathematical Morphology: From Theory to Applications, chap. 3, pp. 81-107. ISTE / J. Wiley \& Sons (2010)

10. Najman, L., Schmitt, M.: Geodesic saliency of watershed contours and hierarchical segmentation. IEEE Transactions on Pattern Analysis and Machine Intelligence 18(12), 1163-1173 (1996)

11. Ore, O.: Theory of equivalence relations. Duke Mathematical Journal 9, 573-627 (1942)

12. Ronse, C.: Partial partitions, partial connections and connective segmentation. Journal of Mathematical Imaging and Vision 32(2), 97-125 (2008). DOI 10.1007/s10851-008-0090-5

13. Ronse, C.: Adjunctions on the lattices of partitions and of partial partitions. Applicable Algebra in Engineering, Communication and Computing 21(5), 343-396 (2010). DOI 10.1007/s00200-010-0129-x

14. Ronse, C.: Orders on partial partitions and maximal partitioning of sets. In: P. Soille, G. Ouzounis, M. Pesaresi (eds.) Proceedings of ISMM 2011, the 10th International Symposium on Mathematical Morphology, Lecture Notes in Computer Science, vol. 6671, pp. 49-60. SpringerVerlag, Berlin (2011)

15. Ronse, C.: Ordering partial partitions for image segmentation and filtering: merging, creating and inflating blocks. Journal of Mathematical Imaging and Vision 49(2), 202-233 (2014). DOI 10.1007/s10851-013-0455-2

16. Ronse, C.: Orders on partial partitions based on block apportioning. Acta Applicandae Mathematicae 141(1), 69-105 (2016). DOI 10.1007/s10440-014-0004-z

17. Ronse, C., Serra, J.: Algebraic foundations of morphology. In: L. Najman, H. Talbot (eds.) Mathematical Morphology: From Theory to Applications, chap. 2, pp. 3580. ISTE / J. Wiley \& Sons (2010)

18. Rota, G.C.: The number of partitions of a set. The American Mathematical Monthly 71(5), 498-504 (1964)

19. Salembier, P., Wilkinson, M.H.F.: Connected operators: A review of region-based morphological image processing techniques. IEEE Signal Processing Magazine 26(6), 136-157 (2009)

20. Serra, J.: Ordre de la construction et segmentations hiérarchiques. Tech. rep., ESIEE/A2SI/IGM (2010)

21. Serra, J.: Grain building ordering. In: P. Soille, G. Ouzounis, M. Pesaresi (eds.) Proceedings of ISMM 2011, the 10th International Symposium on Mathematical Morphology, Lecture Notes in Computer Science, vol. 6671, pp. 37-48. Springer-Verlag, Berlin (2011)

22. Soille, P.: Morphological Image Analysis: Principles and Applications, 2nd edn. Springer, Berlin Heidelberg (2003) 